TRANSACTIONS OF THE

AMERICAN MATHEMATICAL SOCIETY

Volume 349, Number 12, December 1997, Pages 4693-4751

S $0002-9947(97) 01855-2$

\title{
THE JACOBSON RADICAL OF GROUP RINGS OF LOCALLY FINITE GROUPS
}

\author{
D. S. PASSMAN
}

\begin{abstract}
This paper is the final installment in a series of articles, started in 1974, which study the semiprimitivity problem for group algebras $K[G]$ of locally finite groups. Here we achieve our goal of describing the Jacobson radical $\mathcal{J} K[G]$ in terms of the radicals $\mathcal{J} K[A]$ of the group algebras of the locally subnormal subgroups $A$ of $G$. More precisely, we show that if char $K=$ $p>0$ and if $\mathbb{O}_{p}(G)=1$, then the controller of $\mathcal{J} K[G]$ is the characteristic subgroup $\mathbb{S}^{p}(G)$ generated by the locally subnormal subgroups $A$ of $G$ with $A=\mathbb{O}^{p^{\prime}}(A)$. In particular, we verify a conjecture proposed some twenty years ago and, in so doing, we essentially solve one half of the group ring semiprimitivity problem for arbitrary groups. The remaining half is the more difficult case of finitely generated groups.

This article is effectively divided into two parts. The first part, namely the material in Sections 2-6, covers the group theoretic aspects of the proof and may be of independent interest. The second part, namely the work in Sections $7-12$, contains the group ring and ring theoretic arguments and proves the main result. As usual, it is necessary for us to work in the more general context of twisted group algebras and crossed products. Furthermore, the proof ultimately depends upon results which use the Classification of the Finite Simple Groups.
\end{abstract}

\section{INTRODUCTION}

Let $K$ be a field and let $G$ be a multiplicative group. Then the group algebra $K[G]$ is an associative $K$-algebra having the elements of $G$ as a $K$-basis. The addition in $K[G]$ is as expected, and the multiplication is defined distributively using the multiplication in $G$. More generally, a twisted group algebra $K^{t}[G]$ is an associative $K$-algebra having a copy $\bar{G}$ of $G$ as a $K$-basis. Here the multiplication is defined using the formulas

$$
\bar{x} \bar{y}=\tau(x, y) \overline{x y} \quad \text { for all } x, y \in G,
$$

where the twisting function $\tau$ maps $G \times G$ to $K^{\bullet}=K \backslash 0$. Furthermore, the 2cocycle relation satisfied by $\tau$ is precisely equivalent to the associativity of $K^{t}[G]$ (see [P5, Sections 1.1 and 1.2]). As usual, we can assume that $\overline{1}=1$ is the identity element of $K^{t}[G]$.

Of course, $K^{t}[G]$ is an algebra, so it is appropriate to study its ring theoretic properties, and to seek relationships between the group theoretic structure of $G$ and

Received by the editors May 23, 1996.

1991 Mathematics Subject Classification. Primary 16S34; Secondary 16S35, 20F50, 20F24.

Key words and phrases. Group algebras, twisted group algebras, Jacobson radical, semiprimitivity, locally finite groups.

Research supported by NSF Grant DMS-9224662. 
the ring theoretic structure of $K^{t}[G]$. One such theme of particular interest is the semiprimitivity problem. Namely, we wish to determine when $K^{t}[G]$ is semiprimitive, and more generally to find a reasonable description for its Jacobson radical $\mathcal{J} K^{t}[G]$. This problem was studied quite intensely in the 1970s and revisited again in the 1990s (see [P11]). In 1974, the task was essentially split into two. Specifically, it was shown in [P2, Section I] that if one could solve the problem for finitely generated groups and for locally finite groups, then these answers could be combined to handle all groups. The first part still appears to be a rather hopeless task, but the second part always seemed to be more promising. Indeed, the goal of this paper is to complete the work on locally finite groups by verifying a conjecture proposed some twenty years ago. Of course, in so doing, we also solve half of the group ring semiprimitivity problem for arbitrary groups. Note that, if char $K=0$, then one knows that $\mathcal{J} K^{t}[G]=0$ (see [P5, Lemma 7.4.2]), and therefore our concern here is with fields of characteristic $p>0$.

In 1979, the case of locally finite, locally $p$-solvable groups was settled in [P6]. At that time, it appeared that the Classification of Finite Simple Groups (CFSG) would be needed to proceed further, and this has indeed proved to be the case. For example, paper [PZ] uses CFSG directly to handle the nonlinear simple groups, and other papers use it indirectly. In particular, [P9] is based upon the well known result of $[\mathrm{Be}],[\mathrm{Bo}],[\mathrm{HaS}]$ and $[\mathrm{T}]$ which determines all locally finite, infinite simple linear groups, while $[\mathrm{P} 10]$ and the present paper require the classification of the infinite simple, finitary linear groups as given in [H1] and [H2].

This paper builds upon a good deal of earlier work. To start with, references $[\mathrm{P} 8],[\mathrm{P} 9],[\mathrm{P} 10]$ and $[\mathrm{PZ}]$ studied semiprimitivity under certain global assumptions on the locally finite group $G$. Then, papers [P12] and [P13] used the preceding results to deal with the more important local assumptions, and indeed they combined to essentially obtain necessary and sufficient conditions for a twisted group algebra $K^{t}[G]$ of a locally finite group $G$ to be semiprimitive. Here, we complete the cycle by describing the Jacobson radical $\mathcal{J} K^{t}[G]$, and we require the main result of [P13] at two crucial points in the proof. Another important ingredient in this argument is the much earlier work of [P4], which handled the finite extension problem. Unfortunately, that paper restricted its attention to ordinary group algebras, while we need the result here in the twisted context. Furthermore, we also need some of the purely group theoretic aspects of that paper, but in a slightly different form. Thus it is necessary for us to redo some of the work of [P4] in this article. Of course, this affords us the opportunity to shorten and neaten some of the original arguments.

In attempting to describe $\mathcal{J} K^{t}[G]$, we obviously need a mechanism to describe any ideal of the algebra $K^{t}[G]$. One such possibility is to consider the supports of the generators of the ideal. To this end, let $I$ be any fixed ideal of $K^{t}[G]$. If $H \triangleleft G$, then $I \cap K^{t}[H]$ is an ideal of $K^{t}[H]$ which is $G$-stable in the sense that $\bar{g}^{-1}\left(I \cap K^{t}[H]\right) \bar{g}=I \cap K^{t}[H]$ for all $g \in G$. It follows that

$$
K^{t}[G] \cdot\left(I \cap K^{t}[H]\right)=\left(I \cap K^{t}[H]\right) \cdot K^{t}[G]
$$

is a 2-sided ideal of $K^{t}[G]$ contained in $I$, and we say that $H$ controls $I$ if and only if $I=\left(I \cap K^{t}[H]\right) \cdot K^{t}[G]$. In other words, $H$ controls $I$ if and only if $K^{t}[H]$ contains a full set of generators for the ideal. Note that, if $I=L \cdot K^{t}[G]$ for any $G$-stable ideal $L$ of $K^{t}[H]$, then $L=I \cap K^{t}[H]$ and $H$ controls $I$. Now it turns out (see [P5, Lemma 8.1.1]) that the set of controlling subgroups for $I$ is an upwards facing cone with a unique minimal member $\mathcal{C}(I)$, the controller of $I$. Furthermore, if $I$ is a 
characteristic ideal of the ordinary group algebra $K[G]$, then we expect $\mathcal{C}(I)$ to be a characteristic subgroup of $G$ ([P5, Lemma 8.1.2]). Thus, one approach to describing ideals in $K^{t}[G]$ is to find controlling subgroups. In this paper, we essentially find $\mathcal{C}\left(\mathcal{J} K^{t}[G]\right)=C$ and we obtain an adequate description of the intersection ideal $\mathcal{J} K^{t}[G] \cap K^{t}[C]$ when $G$ is locally finite.

To see what we might expect for an answer, let us briefly consider a related, but considerably easier problem. For any $\operatorname{ring} R$, let $\mathcal{N} R$ denote its nilpotent radical, namely the join of all its nilpotent ideals. Obviously, $\mathcal{N} R$ is a characteristic nil ideal of $R$ and, of course, it need not be nilpotent. Next, for any group $G$, we let $\Delta^{p}(G)$ denote its characteristic subgroup generated by all finite $D \triangleleft G$ with $D=D^{p}$. Here we write $D^{p}$ as an abbreviation for $\mathbb{O}^{p^{\prime}}(D)$, namely the subgroup of $D$ generated by its $p$-elements. The following result is proved by a coset counting argument known as the Delta Method (see [P5, Theorem 8.1.9] or [P8, Proposition 2.8] for somewhat simpler proofs).

Proposition 1.1. [P1] Let $K^{t}[G]$ be a twisted group algebra of the arbitrary group $G$ over a field $K$ of characteristic $p>0$. Then we have

i. $\mathcal{N} K^{t}[G]=\mathcal{J} K^{t}\left[\Delta^{p}(G)\right] \cdot K^{t}[G]$.

ii. $\mathcal{J} K^{t}\left[\Delta^{p}(G)\right]=\bigcup_{D \in \mathfrak{D}} \mathcal{J} K^{t}[D]$, where $\mathfrak{D}$ is the set of all finite normal subgroups $D$ of $G$ with $D=D^{p}$.

iii. $\mathcal{N} K^{t}[G]=0$ if and only if $\mathcal{J} K^{t}[D]=0$ for all $D \in \mathfrak{D}$. In particular, if $\mathfrak{D}$ consists of just the identity group 1 , then $\mathcal{N} K^{t}[G]=0$.

Note that, if $K^{t}[G]=K[G]$ is an ordinary group algebra or if $K$ is a perfect field, then $\mathcal{C}\left(\mathcal{N} K^{t}[G]\right)=\Delta^{p}(G)$ and, in particular, $\mathcal{N} K^{t}[G]=0$ if and only if $\Delta^{p}(G)=1$. We will prove this controller observation in Section 12; it was previously known only for ordinary group algebras (see [P5, Theorem 8.1.9]). As we will see, twisted group algebras over imperfect fields can exhibit rather anomalous behavior. Indeed, when this occurs, the controller $\mathcal{C}\left(\mathcal{N} K^{t}[G]\right)$ can be considerably smaller than $\Delta^{p}(G)$.

For the remainder of this introduction we consider only locally finite groups. As we indicated, our goal is to describe the Jacobson radical $\mathcal{J} K^{t}[G]$ in a manner analogous to that given for $\mathcal{N} K^{t}[G]$ above. For this, it turns out that the finite normal subgroups which occur in Proposition 1.1 must be replaced by the locally subnormal subgroups of $G$, which are defined as follows. Let $A$ be a finite subgroup of $G$. Then we say that $A$ is locally subnormal in $G$, and write $A \operatorname{lsn} G$, if $A$ is subnormal in all finite subgroups $B \subseteq G$ which contain $A$. Furthermore, we let $\mathbb{S}^{p}(G)$ be the characteristic subgroup of $G$ generated by all $A \operatorname{lsn} G$ with $A=A^{p}$, so that $\mathbb{S}^{p}(G)$ plays a role similar to that of $\Delta^{p}(G)$. With this notation in hand, we can now state our main result.

Theorem 1.2. Let $K^{t}[G]$ be a twisted group algebra of the locally finite group $G$ over a field $K$ of characteristic $p>0$. If $\mathbb{O}_{p}(G)=1$, then

i. $\mathcal{J} K^{t}[G]=\mathcal{J} K^{t}\left[\mathbb{S}^{p}(G)\right] \cdot K^{t}[G]$.

ii. $\mathcal{J} K^{t}\left[\mathbb{S}^{p}(G)\right]=\bigcup_{A \in \mathfrak{S}} \mathcal{J} K^{t}[A]$, where $\mathfrak{S}$ is the set of all locally subnormal subgroups $A$ of $G$ with $A=A^{p}$.

iii. $\mathcal{J} K^{t}[G]=0$ if and only if $\mathcal{J} K^{t}[A]=0$ for all $A \in \mathfrak{S}$. In particular, if $\mathfrak{S}$ consists of just the identity group 1 , then $\mathcal{J} K^{t}[G]=0$.

iv. $\mathcal{J} K^{t}[G]$ is a prime ideal of $K^{t}[G]$ when $G$ has no nonidentity finite normal subgroups. 
Note that the assumption $\mathbb{O}_{p}(G)=1$ in the above is easily overcome by merely modding out $\mathbb{O}_{p}(G)$. Indeed, if we define $\mathbb{T}^{p}(G) \supseteq \mathbb{O}_{p}(G)$ by

$$
\mathbb{T}^{p}(G) / \mathbb{O}_{p}(G)=\mathbb{S}^{p}\left(G / \mathbb{O}_{p}(G)\right),
$$

then we have the following immediate consequence.

Corollary 1.3. If $K^{t}[G]$ is a twisted group algebra of the locally finite group $G$ over a field $K$ of characteristic $p>0$, then

i. $\mathcal{J} K^{t}[G]=\mathcal{J} K^{t}\left[\mathbb{T}^{p}(G)\right] \cdot K^{t}[G]$.

ii. $\mathcal{J} K^{t}[G]=0$ if and only if $\mathcal{J} K^{t}[A]=0$ for all locally subnormal subgroups $A$ of $G$ with $A=A^{p}$. In particular, if the only such group $A$ is the identity 1 , then $\mathcal{J} K^{t}[G]=0$.

Furthermore, we can give a reasonably accurate description of both $\mathcal{J} K^{t}[G]$ and $\mathcal{J} K^{t}\left[\mathbb{T}^{p}(G)\right]$. Indeed, if either $K^{t}[G]$ is an ordinary group algebra or if $K$ is a perfect field, then $K^{t}\left[\mathbb{O}_{p}(G)\right] \cong K\left[\mathbb{O}_{p}(G)\right]$ has Jacobson radical equal to its augmentation ideal, and hence this radical has codimension 1. Consequently, if we set $J=\mathcal{J} K^{t}\left[\mathbb{O}_{p}(G)\right] \cdot K^{t}[G]$, then $J \subseteq \mathcal{J} K^{t}[G]$ and $K^{t}[G] / J \cong K^{t^{\prime}}\left[G / \mathbb{O}_{p}(G)\right]$, where the latter is a naturally defined twisted group algebra of $G / \mathbb{O}_{p}(G)$. Of course, $G / \mathbb{O}_{p}(G)$ has no nontrivial normal $p$-subgroups and $\mathcal{J} K^{t}[G] / J \cong \mathcal{J} K^{t^{\prime}}\left[G / \mathbb{O}_{p}(G)\right]$, so Theorem 1.2 quickly yields the result. Note that, under these assumptions, it follows that $\mathcal{C}\left(\mathcal{J} K^{t}[G]\right)=\mathbb{T}^{p}(G)$. On the other hand, if $K$ is not perfect, then the controller of the Jacobson radical can be properly smaller than $\mathbb{T}^{p}(G)$.

We close this section with a few personal comments. To start with, I would like to thank Professor Alex Zalesskiü for rekindling my interest in the group ring semiprimitivity problem. He visited Madison in 1990 and told me of his ideas concerning infinite simple groups. This led to our joint paper [PZ] and profoundly changed the direction of my research. Next, I would like to thank Professors Jon Hall, Dick Phillips and Ulrich Meierfrankenfeld. Their results on finitary linear groups are crucial to the arguments here, and they were kind enough to give me preliminary copies of their papers and to promptly answer my email questions. Finally, some of the work on this paper was done during a delightful month-long stay at the Université Pierre et Marie Curie (Paris VI) this past summer (1995). Both my wife and I would like to thank Professor Marie-Paule Malliavin for the invitation and for her kind hospitality.

\section{Subnormality in Finite Groups}

We start by gathering together a number of fairly standard results on subnormality in finite groups. These will be needed to study local subnormality in locally finite groups. For the time being, all groups considered are assumed to be finite. As usual, $A \triangleleft G$ indicates that $A$ is a normal subgroup of $G$, and $A \triangleleft \triangleleft G$ indicates that $A$ is subnormal. Recall that a property $(*)$ is said to be normally persistent if the product of two normal $(*)$-subgroups is a $(*)$-group. Similarly, $(*)$ is $s u b$ normally persistent if the group generated by any two subnormal (*)-subgroups is also a (*)-group. Some key observations are as follows (see [W1] or [Za, pages 194 and 246]).

Lemma 2.1. Let $G$ be a finite group.

i. If $A, B \triangleleft \triangleleft G$, then $A \cap B$ and $\langle A, B\rangle$ are also subnormal in $G$.

ii. Normal persistence implies subnormal persistence. 
By applying part (ii) of the preceding lemma to the normal closure of the subnormal subgroups given below, we obtain

Lemma 2.2. Let $G$ be a finite group.

i. If $\pi$ is a set of primes, then any subnormal $\pi$-subgroup of $G$ is contained in $\mathbb{O}_{\pi}(G)$, the largest normal $\pi$-subgroup of $G$.

ii. Any subnormal nilpotent subgroup of $G$ is contained in the Fitting subgroup $\mathbb{F}(G)$, namely the largest normal nilpotent subgroup of $G$.

iii. If $A$ is a solvable subnormal subgroup of $G$, then its normal closure $A^{G}$ is a solvable normal subgroup of $G$.

Recall that if $A$ and $B$ are subgroups of $G$, then their commutator group $[A, B]$ is the subgroup of $G$ generated by all commutators $[a, b]=a^{-1} b^{-1} a b$ with $a \in A$ and $b \in B$. It is clear that $[A, B]=[B, A]$ and that $[A, B]=1$ if and only if $A$ and $B$ centralize each other. Furthermore, if $B$ normalizes $A$, then $[A, B] \subseteq A$, and if $C$ normalizes $A$ and $B$, then $C$ normalizes $[A, B]$. More substantial observations are stated below. Note that the triple commutator $[A, B, C]$ is defined by associating to the left, so that $[A, B, C]=[[A, B], C]$.

Lemma 2.3. Let $A, B$, and $C$ be subgroups of $G$ and let $N \triangleleft G$.

i. $A$ and $B$ normalize $[A, B]$.

ii. If $[A, B, C] \subseteq N$ and $[B, C, A] \subseteq N$, then $[C, A, B] \subseteq N$.

Note that part (ii) above is usually called the Three Subgroups Theorem. For a proof of the lemma see [Hu, Hilfssatz III.1.6 and Satz III.1.10]. Now if $V, A \subseteq G$, we define the multicommutators $[V, A]_{n}$ inductively by $[V, A]_{0}=V$ and $[V, A]_{n+1}=$ $\left[[V, A]_{n}, A\right]$. In other words,

$$
[V, A]_{n}=[V, \underbrace{A, A, \ldots, A}_{n \text { times }}]
$$

with the latter expression associated to the left. Note that if $V$ and $A$ are abstract groups with $A$ acting on $V$, then we can use the same commutator notation by working in the semidirect product $V \rtimes A$. Of course, $[v, a]=v^{-1} a^{-1} v a=v^{-1} v^{a}$ can be computed directly in $V$ without recourse to the larger group $V \rtimes A$. In any case, we say that $A$ is unipotent on $V$ if $[V, A]_{n}=1$ for some integer $n$.

Lemma 2.4. Let $A$ and $B$ be subgroups of $G$ and let $G$ act on $V$.

i. If $[V, A]_{n}=1$ with $n \geq 1$, then $\left[V, A^{(n-1)}\right]=1$ where $A^{(n-1)}$ denotes the $(n-1)$ st derived subgroup of $A$.

ii. Suppose that $[V, A]_{n}=1$ and $[V, B]_{m}=1$ for some integers $m, n \geq 0$. If $A$ normalizes $B$, then $[V, A B]_{m n}=1$.

iii. Let $A$ and $B$ be subnormal subgroups of $G$. If $A$ and $B$ are unipotent on $V$, then $\langle A, B\rangle$ is also unipotent on $V$.

Proof. (i) This is a standard application of the Three Subgroups Theorem and is proved by induction on $n$, the case $n=1$ being trivial. If $n \geq 2$, then $[[V, A], A]_{n-1}=1$, so we conclude by induction that $\left[V, A, A^{(n-2)}\right]=1$ and hence that

$$
\left[A^{(n-2)}, V, A^{(n-2)}\right]=\left[V, A^{(n-2)}, A^{(n-2)}\right] \subseteq\left[V, A, A^{(n-2)}\right]=1 .
$$


Consequently, Lemma 2.3(ii) yields

$$
\left[V, A^{(n-1)}\right]=\left[A^{(n-1)}, V\right]=\left[A^{(n-2)}, A^{(n-2)}, V\right]=1,
$$

and the result follows.

(ii) If we set $V_{i}=[V, B]_{i}$, then Lemma 2.3(i) and the hypothesis imply that

$$
1=V_{m} \triangleleft V_{m-1} \triangleleft \cdots \triangleleft V_{1} \triangleleft V_{0}=V
$$

and that each $V_{i}$ is $B$-stable. Furthermore, since $A$ normalizes both $B$ and $V$, it follows that each $V_{i}$ is $A$-stable. Thus $A B$ acts on $\bar{V}_{i}=V_{i} / V_{i+1}$ and, since $B$ acts trivially on this quotient and $\left[V_{i}, A\right]_{n} \subseteq[V, A]_{n}=1$, we have

$$
\left[\bar{V}_{i}, A B\right]_{n}=\left[\bar{V}_{i}, A\right]_{n}=\overline{\left[V_{i}, A\right]_{n}}=1 \text {. }
$$

In other words, $\left[V_{i}, A B\right]_{n} \subseteq V_{i+1}$ and therefore $[V, A B]_{m n}=\left[V_{0}, A B\right]_{m n} \subseteq V_{m}=1$.

(iii) Part (ii) implies that the property of being unipotent on $V$ is normally persistent. Thus, by Lemma 2.1(ii), this property is also subnormally persistent.

It is clear that the preceding commutator observations hold without any finiteness assumption. Now we combine the concept of subnormality with that of a unipotent action. For convenience, we use $A^{(\infty)}$ to denote the last term of the derived series of the finite group $A$.

Lemma 2.5. Let $G$ be a finite group and let $A \triangleleft \triangleleft G$.

i. $[G, A]_{n} \subseteq A$ for some integer $n$.

ii. If $A$ normalizes $N$ and $N \cap A \subseteq \mathbb{Z}(A)$, then $A^{(\infty)}$ centralizes $N$.

Proof. (i) By definition of subnormality there exists a subnormal series

$$
A=A_{n} \triangleleft A_{n-1} \triangleleft \cdots \triangleleft A_{1} \triangleleft A_{0}=G,
$$

and observe that $\left[A_{i}, A\right] \subseteq\left[A_{i}, A_{i+1}\right] \subseteq A_{i+1}$ for each $i<n$. Thus $[G, A]_{j}=$ $\left[A_{0}, A\right]_{j} \subseteq A_{j}$ for all $j \leq n$ and, in particular, $[G, A]_{n} \subseteq A_{n}=A$.

(ii) Since $A$ normalizes $N$ and $N \subseteq G$, part (i) implies that $[N, A]_{n} \subseteq N \cap A \subseteq$ $\mathbb{Z}(A)$. Thus $[N, A]_{n+1} \subseteq[\mathbb{Z}(A), A]=1$, and Lemma 2.4(i) implies that $\left[N, A^{(\infty)}\right] \subseteq$ $\left[N, A^{(n)}\right]=1$.

We say that a finite group $A$ is quasi-simple if $A=A^{\prime}$ and $A / \mathbb{Z}(A)$ is nonabelian simple. In other words, $A$ is a nontrivial homomorphic image of the Schur central cover of a nonabelian simple group. Such groups come into play because of

Lemma 2.6. Let $N$ be a maximal normal subgroup of the finite group $A$ and assume that $[N, A]_{n}=1$ for some integer $n$. Then either $A$ is nilpotent or $A=N A^{(\infty)}$ with $N$ nilpotent and $A^{(\infty)}$ quasi-simple.

Proof. Since $[N, N]_{n} \subseteq[N, A]_{n}=1$, it is clear that $N$ is nilpotent. Furthermore, if $A / N$ is abelian, then $A^{\prime}=[A, A] \subseteq N$, so $[A, A]_{n+1}=[[A, A], A]_{n} \subseteq[N, A]_{n}=$ 1 and $A$ is also nilpotent. On the other hand, if $A / N$ is nonabelian, then the maximality of $N$ implies that this quotient is nonabelian simple. In particular, $(A / N)^{(\infty)}=A / N$, so it follows that $A=N B$ where $B=A^{(\infty)}$. Note that $B=B^{\prime}$ and that $B /(B \cap N) \cong B N / N=A / N$ is nonabelian simple. Finally, $[B \cap N, B]_{n} \subseteq$ $[N, A]_{n}=1$, so Lemma 2.4(i) implies that $[B \cap N, B]=\left[B \cap N, B^{(n-1)}\right]=1$. In other words, $B \cap N \subseteq \mathbb{Z}(B)$ and $B$ is quasi-simple.

Key properties of quasi-simple groups are as follows. 
Lemma 2.7. Let $A$ be a quasi-simple group.

i. If $N \triangleleft \triangleleft A$, then either $N=A$ or $N \subseteq \mathbb{Z}(A)$. In particular, $N \triangleleft A$ and $A / N$ is either quasi-simple or trivial.

ii. If $A \triangleleft \triangleleft G$ and $B \triangleleft \triangleleft G$, then $A$ normalizes $B$. Indeed, if $B$ is solvable or if $B \neq A$ is quasi-simple, then $A$ centralizes $B$.

Proof. (i) Let $Z=\mathbb{Z}(A)$ and suppose that $N \nsubseteq Z$. Then $N Z / Z$ is a nontrivial subnormal subgroup of the simple group $A / Z$, so $N Z / Z=A / Z$ and $N Z=A$. Consequently, $A=[A, A]=[N Z, N Z]=[N, N] \subseteq N$, and $A=N$ as required.

(ii) Let $A, B \triangleleft \triangleleft G$ and suppose first that $B$ is solvable. Then Lemma 2.2(iii) implies that the normal closure $B^{G}$ is also solvable, and hence $B^{G} \cap A \subseteq \mathbb{Z}(A)$ by (i) above. Thus, by Lemma 2.5 (ii), $\left[B^{G}, A\right]=\left[B^{G}, A^{(\infty)}\right]=1$, and therefore $A$ centralizes $B^{G}$. In particular, $A$ centralizes and hence normalizes $B$.

Now let $B$ be an arbitrary subnormal subgroup of $G$. We show that $A$ normalizes $B$ by induction on $|B|$, the result being trivial if $B=1$. We may of course assume that $G=\langle A, B\rangle$, and we first consider the case where $B$ is not simple. To this end, let $C$ be a proper normal subgroup of $B$. Since $C \triangleleft \triangleleft G$ and $|C|<|B|$, it follows by induction that $C$ is normalized by $A$. Thus $C \triangleleft\langle A, B\rangle=G$ and we let ${ }^{-}: G \rightarrow G / C$ be the natural epimorphism. Then $\bar{B}=B / C \triangleleft \triangleleft \bar{G}$ and also $\bar{A} \triangleleft \triangleleft \bar{G}$. Furthermore, by (i) above, $\bar{A}$ is either equal to 1 or quasi-simple. Thus, since $|\bar{B}|<|B|$, induction implies that $\bar{A}$ normalizes $\bar{B}$ and hence that $\bar{B} \triangleleft\langle\bar{A}, \bar{B}\rangle=\bar{G}$. But $B \supseteq C$, so we conclude that $B \triangleleft\langle A, B\rangle=G$, and this case is proved.

Next, we assume that $B$ is a nonidentity simple group. Since the abelian case has already been considered, we may further suppose that $B$ is nonabelian simple. If $B \subseteq A$, then $B \triangleleft \triangleleft A$ and therefore (i) above implies that $A$ normalizes $B$. On the other hand, if $B \nsubseteq A$, then $A \neq G=\langle A, B\rangle$, so $A \triangleleft \triangleleft G$ implies that $A \subseteq H \triangleleft G$ with $H$ a proper normal subgroup of $G$. Certainly $B \nsubseteq H$ since $G=\langle A, B\rangle \supset H$, and therefore $B \neq B \cap H \triangleleft B$. Thus, since $B$ is simple, we have $B \cap H=1$, and Lemma 2.5(ii) implies that $[H, B]=\left[H, B^{(\infty)}\right]=1$. Consequently, $H$ centralizes $B$, and it follows that $A \subseteq H$ centralizes and hence normalizes $B$.

We have therefore shown that if $B \triangleleft \triangleleft G$, then $A$ normalizes $B$. Furthermore, if $B$ is solvable then $A$ centralizes $B$. It remains to consider the action of $A$ on $B$ when the latter group is quasi-simple. Suppose first that $A \cap B$ is nonabelian. Since $A \cap B \triangleleft \triangleleft A$, (i) implies that $A \cap B=A$, and similarly, since $A \cap B \triangleleft \triangleleft B$, we have $A=A \cap B=B$. Consequently, if $A \neq B$, then we must have $A \cap B$ abelian and hence $A \cap B \subseteq \mathbb{Z}(A)$. Finally, since $A$ normalizes $B$ and $A \triangleleft \triangleleft\langle A, B\rangle$, Lemma 2.5(ii) yields $[B, A]=\left[B, A^{(\infty)}\right]=1$, and the lemma is proved.

The following technical result will be used when we study the generalized Fitting subgroup.

Lemma 2.8. Let $G$ be a finite group generated by subnormal subgroups which are either nilpotent or quasi-simple. If $N \triangleleft \triangleleft G$, then $N$ is also generated by subnormal subgroups which are either nilpotent or quasi-simple.

Proof. We proceed by induction on $|G|$ and we let $F$ denote the Fitting subgroup of $G$. Then $F$ is a normal nilpotent subgroup of $G$, so $[G, F] \subseteq F$ and therefore $[G, F]_{n}=1$ for some integer $n$. Since all nilpotent subnormal subgroups of $G$ are contained in $F$, we can write $G=\left\langle F, A_{1}, A_{2}, \ldots, A_{k}\right\rangle$ where the $A_{i}$ are all the subnormal quasi-simple subgroups of $G$. Note that, if $S$ is a solvable normal subgroup of $G$, then each $A_{i}$ centralizes $S$ by the preceding lemma. Hence $[S, G]_{n}=$ 
$[S, F]_{n} \subseteq[G, F]_{n}=1$. In particular, $[S, S]_{n}=1$, so $S$ is nilpotent and $S \subseteq F$. It now follows that any solvable subnormal subgroup of $G$ is also nilpotent.

Let $N$ be any subnormal subgroup of $G$. If $N$ is solvable, then $N$ is nilpotent by the above, and the result is clear. Thus we may suppose that $N$ is not solvable and we choose $A \subseteq N$ to be a minimal nonsolvable subnormal subgroup of $N$. Then $A \triangleleft \triangleleft G$ and it is clear that $A=A^{\prime}$ since otherwise $A^{\prime}$ would be solvable. Now let $B$ be a maximal normal subgroup of $A$. Again, $B \triangleleft \triangleleft N$, so the minimality of $A$ implies that $B$ is solvable and that $A / B$ is nonabelian simple. Furthermore, $S=B^{G}$ is a solvable normal subgroup of $G$, so the remarks of the previous paragraph imply that $[B, A]_{n} \subseteq[S, G]_{n}=1$. Therefore $A$ is quasi-simple by Lemma 2.6 and, for convenience, we suppose that $A=A_{k}$.

Finally, let $C=\left\langle F, A_{1}, \ldots, A_{k-1}\right\rangle$. Then $G=\left\langle C, A_{k}\right\rangle$ and $A_{k}$ centralizes $C$ by Lemma 2.7(ii). Thus $C \triangleleft G$ and $G=C A_{k}$. In addition, we note that $G \neq C$ since otherwise $A_{k}$ would be central in $G$. Now, $G=C A_{k} \supseteq N \supseteq A_{k}$, so $N=(C \cap N) A_{k}$. Furthermore, $C$ has the same structure as $G$ and $C \cap N \triangleleft \triangleleft C$. Thus, by induction, $C \cap N$ is generated by subnormal subgroups which are either nilpotent or quasisimple and hence the same is true for $N=(C \cap N) A_{k}$ since $C \cap N \triangleleft N$.

Recall that a finite group $G$ is said to be semisimple if it is the direct product of nonabelian simple groups. Obviously, any such group is generated by its subnormal nonabelian simple subgroups. Conversely, since any two distinct subnormal nonabelian simple subgroups commute, by Lemma 2.7(ii), it follows that any group generated by such subgroups is necessarily semisimple. As a consequence, we have part (i) of the following result, and part (ii) is, of course, routine.

Lemma 2.9. Let $G$ be a finite group.

i. The property of being semisimple is subnormally persistent.

ii. If $G=M_{1} \times M_{2} \times \cdots \times M_{k}$ is semisimple with each $M_{i}$ nonabelian simple, then any subnormal subgroup of $G$ is a partial direct product of the $M_{i} s$ and hence it is normal. In particular, $\left\{M_{1}, M_{2}, \ldots, M_{k}\right\}$ is the set of minimal normal subgroups of $G$.

Finally, we mention a $\pi$ analog of Lemma 2.7. For convenience, if $G$ is any group and if $\pi$ is any set of primes, then we write $G^{\pi}$ for $\mathbb{O}^{\pi^{\prime}}(G)$, the characteristic subgroup of $G$ generated by its $\pi$-elements. Of course, if $\pi$ consists of just one prime $p$, then we denote this subgroup by $G^{p}$.

Lemma 2.10. Let $A$ and $B$ be subnormal subgroups of $G$. If $A$ is a $\pi^{\prime}$-group and if $B=B^{\pi}$, then $A$ normalizes $B$.

Proof. Since the normal closure of $A$ is also a $\pi^{\prime}$-group, we can assume that $A \triangleleft G$. In addition, we may suppose that $G=\langle A, B\rangle=A B$, and we proceed by induction on $|G|$. Specifically we show that, under these conditions, $G^{\pi}=B^{\pi}=B$ and consequently $B$ is normal in $G$. Now, this fact is obvious if $B=G$, so suppose that $B$ is a proper subgroup. Then, since $B \triangleleft \triangleleft G$, there exists a proper normal subgroup $H$ of $G$ with $B \subseteq H$. Note that $G=A B=A H$, so $G / H \cong A /(A \cap H)$ is a $\pi^{\prime}$-group and hence $G^{\pi}=H^{\pi}$. Furthermore, $A B \supseteq H \supseteq B$, so $H=(H \cap A) B$ and therefore $H$ has the same structure as $G$. By induction, we conclude that $H^{\pi}=B^{\pi}=B$ and the lemma is proved. 


\section{Finite Group Actions}

In this section, we continue our study of finite groups and obtain some rather technical lemmas which will be needed for later applications. Specifically, we offer a simplified approach to the group theoretic results of [P4]. We start with an observation on permutation groups.

Lemma 3.1. Let $G$ be a finite group acting primitively as permutations on a set $\Omega$ and suppose that $A \triangleleft \triangleleft G$.

i. If $A$ is nontrivial in its action, then there exists $x \in G$ such that the group $\left\langle A, A^{x}\right\rangle$ fixes no point of $\Omega$.

ii. If $a \in A$ moves $k>0$ points of $\Omega$, then $|\Omega| \leq 2 k|A|$.

Proof. (i) We can assume that $G$ acts faithfully on $\Omega$ and therefore, by hypothesis, $A \neq 1$. Now let $B \neq 1$ be a minimal subnormal subgroup of $G$ contained in $A$ and set $S=B^{G}$. Since $S \triangleleft G$ and $G$ is primitive on $\Omega$, it follows that $S$ is transitive. In particular, if $1 \neq N \triangleleft S$, then $N$ fixes no point of $\Omega$. As usual, there are two cases to consider.

Suppose first that $B$ is nonabelian simple. Then, by Lemma $2.9, S$ is semisimple, and $B \triangleleft S$. In particular, the preceding remarks imply that $B$ fixes no point of $\Omega$, so the same is true of $A \supseteq B$, and we can take $x=1$ in the conclusion.

The remaining possibility is that $|B|=q$ for some prime $q$. In this case, $S$ is a $q$-group and we let $Z$ denote its center. Since any subgroup of $Z$ is normal in $S$, the remarks of the first paragraph imply that every nonidentity element of $Z$ acts without fixed points on $\Omega$. Consequently, if $S$ is abelian, then $B \subseteq S=Z$ fixes no point of $\Omega$ and therefore the same is true of $A$.

On the other hand, if $S$ is nonabelian, then its second center $Z_{2}$ is properly larger than $Z$. Note that $Z_{2} \triangleleft G$ and that $B$ does not centralize $Z_{2}$ since otherwise $B^{G}=S$ would also centralize this group. Thus we can choose $x \in Z_{2}$ and $b \in B$ with $[b, x] \neq 1$. But then $[b, x] \in\left\langle B, B^{x}\right\rangle \cap Z$, so $\left\langle B, B^{x}\right\rangle$ does not have a fixed point in $\Omega$ and therefore the same is true of the larger group $\left\langle A, A^{x}\right\rangle$.

(ii) Let $C=\langle a\rangle^{A}$ be the normal closure of $\langle a\rangle$ in $A$. Then $C$ is generated by at most $|A|$ conjugates of $a$ and, since $a$ moves $k$ points of $\Omega$, it follows that $C$ moves at most $k|A|$ points. Furthermore, note that $C \triangleleft \triangleleft G$ and that $C$ acts nontrivially on $\Omega$ since $k>0$. Thus, by (i) above, there exists $x \in G$ such that $\left\langle C, C^{x}\right\rangle$ has no fixed points. But $\left\langle C, C^{x}\right\rangle$ moves at most $2 k|A|$ points, and consequently $|\Omega| \leq 2 k|A|$.

Not surprisingly, a similar result holds for linear groups.

Lemma 3.2. Let $G$ be a finite group which acts in a primitive manner on the elementary abelian group $W$ and suppose that $A \triangleleft \triangleleft$.

i. If $A$ acts nontrivially, then there exists $x \in G$ with $\mathbb{C}_{W}\left(\left\langle A, A^{x}\right\rangle\right)=1$.

ii. If $a \in A$ with $\left|W: \mathbb{C}_{W}(a)\right|=k>1$, then $|W| \leq k^{2|A|}$.

Proof. (i) We can assume that $G$ acts faithfully on $W$ and therefore, by hypothesis, $A \neq 1$. Now let $B \neq 1$ be a minimal subnormal subgroup of $G$ contained in $A$ and set $S=B^{G}$. Since $S \triangleleft G$ and since $W$ is a primitive $G$-module, it follows that the restriction of $W$ to $S$ is homogeneous. In other words, $W_{S} \cong U \oplus U \oplus \cdots \oplus U$, where $U$ is an irreducible $S$-module. Thus $W_{S}$ is completely reducible and $S$ acts faithfully on $U$. Now if $1 \neq N \triangleleft S$, then $S$ acts on $\mathbb{C}_{W}(N)$ and consequently this $S$-submodule of $W$ is isomorphic to a direct sum of copies of $U$. But $N$ acts trivially 
on $\mathbb{C}_{W}(N)$ and $S$ is faithful on $U$, so it follows that no $U$ summands can occur. In other words, we must have $\mathbb{C}_{W}(N)=1$.

As in the proof of Lemma 3.1, there are now two cases to consider. Suppose first that $B$ is nonabelian simple. Then $S$ is semisimple and $B \triangleleft S$, so the preceding remarks imply that $1=\mathbb{C}_{W}(B) \supseteq \mathbb{C}_{W}(A)$, and we can take $x=1$ in the conclusion.

The remaining possibility is that $|B|=q$ for some prime $q$. In this case, $S$ is a $q$-group and we let $Z$ denote its center. Since any subgroup of $Z$ is normal in $S$, the remarks of the first paragraph imply that every nonidentity element of $Z$ has trivial centralizer in $W$. In particular, if $S$ is abelian, then $B \subseteq S=Z$ yields $1=\mathbb{C}_{W}(B) \supseteq \mathbb{C}_{W}(A)$, and the result again follows with $x=1$.

On the other hand, if $S$ is nonabelian, then its second center $Z_{2}$ is properly larger than $Z$. Furthermore, $Z_{2} \triangleleft G$ and $B$ does not centralize $Z_{2}$ since $B^{G}=S$ does not centralize this group. Thus we can choose $x \in Z_{2}$ and $b \in B$ with $[b, x] \neq 1$. But then $[b, x] \in\left\langle B, B^{x}\right\rangle \cap Z$, so $\left\langle B, B^{x}\right\rangle$ has trivial centralizer in $W$ and therefore the same is true of the larger group $\left\langle A, A^{x}\right\rangle$.

(ii) Let $C=\langle a\rangle^{A}$ be the normal closure of $\langle a\rangle$ in $A$. Then $C$ is generated by at most $|A|$ conjugates of $a$ and, since $\left|W: \mathbb{C}_{W}(a)\right|=k$, it follows that $\left|W: \mathbb{C}_{W}(C)\right| \leq$ $k^{|A|}$. Furthermore, $C \triangleleft \triangleleft G$ and $C$ acts nontrivially on $W$ since $k \geq 2$. Thus, by (i) above, there exists $x \in G$ such that $\left\langle C, C^{x}\right\rangle$ has trivial centralizer in $W$. But then

$$
|W|=\left|W: \mathbb{C}_{W}\left(\left\langle C, C^{x}\right\rangle\right)\right| \leq\left|W: \mathbb{C}_{W}(C)\right|^{2} \leq k^{2|A|},
$$

so the lemma is proved.

We will need the following elementary observation. For later applications, we allow the $H$-module $W$ to be infinite.

Lemma 3.3. Let $H$ be a finite group and let $W$ be a possibly infinite group acted upon by $H$ with $\left|W: \mathbb{C}_{W}(H)\right|=k<\infty$. Suppose that $W=\prod_{i} W_{i}$ is the direct product of isomorphic subgroups $W_{i}$ and that, in its action on $W, H$ permutes these factors in a nontrivial manner. For convenience, if $\mathcal{O}$ is any $H$-orbit of $W_{i} s$, then we write $W_{\mathcal{O}}$ for the finite direct product of these factors.

i. Each $W_{i}$ is finite of order $\leq k$ and each $W_{\mathcal{O}}$ is an $H$-stable finite subgroup of $W$ of order $\leq k^{2}$.

ii. $W=\prod W_{\mathcal{O}}$, where the direct product is taken over all orbits $\mathcal{O}$.

iii. $H$ fails to centralize at most $k$ of the $W_{\mathcal{O}}$ and at most $k^{2}$ of the $W_{i}$.

Proof. Let $\mathcal{O}=\left\{W_{1}, W_{2}, \ldots, W_{r}\right\}$ be any $H$-orbit of $W_{i}$ s with $r \geq 2$. Since $H$ is transitive on $\mathcal{O}$, it follows that any element of $\mathbb{C}_{W_{\mathcal{O}}}(H)$ is uniquely determined by its projection to the $W_{1}$-factor. In other words, $\mathbb{C}_{W_{\mathcal{O}}}(H)$ is disjoint from the product $W_{2} \times W_{3} \times \cdots \times W_{r}$ and therefore, since all $W_{i}$ are isomorphic, we have

$$
k \geq\left|W: \mathbb{C}_{W}(H)\right| \geq\left|W_{\mathcal{O}}: \mathbb{C}_{W_{\mathcal{O}}}(H)\right| \geq\left|W_{1}\right|^{r-1} \geq\left|W_{1}\right| .
$$

Consequently, $\left|W_{\mathcal{O}}\right|=\left|W_{1}\right|^{r} \leq\left|W_{1}\right|^{2(r-1)} \leq k^{2}$ and $r \leq 1+\log _{2} k \leq k$.

Now the hypothesis of the lemma implies that such an orbit $\mathcal{O}$ exists with $r \geq 2$, and therefore we conclude from the above that $\left|W_{i}\right| \leq k$ for all subscripts $i$. With this, parts (i) and (ii) clearly follow. Finally, since $\mathbb{C}_{W}(H)=\prod \mathbb{C}_{W_{\mathcal{O}}}(H)$, we have $\left|W: \mathbb{C}_{W}(H)\right|=\prod\left|W_{\mathcal{O}}: \mathbb{C}_{W_{\mathcal{O}}}(H)\right|$ and therefore $H$ can act nontrivially on at most $\log _{2} k \leq k$ of the factors $W_{\mathcal{O}}$. Furthermore, each such $W_{\mathcal{O}}$ is a product of at most $k$ factors $W_{i}$, and thus $H$ can act nontrivially on at most $k^{2}$ of these terms. 
For our purposes, it is appropriate to extend the notion of an irreducible representation to allow for nonabelian modules. Specifically, if $G$ acts on the finite group $W$, then we say that this action is irreducible if there are no proper $G$-stable normal subgroups of $W$. In this situation, $W$ has no proper characteristic subgroups, so $W$ is either an elementary abelian $q$-group for some prime $q$, or it is semisimple and in fact the direct product of isomorphic nonabelian simple groups. It is with this understanding that we state

Lemma 3.4. There exist two integer valued functions $\alpha^{\prime}$ and $\beta^{\prime}$ with the following property: Let $A$ and $H$ be subgroups of $G$ with $A \triangleleft \triangleleft, G=H^{G}$ and $[A, H]=A$. Suppose $G$ acts irreducibly on the finite group $W$ and that $\left|W: \mathbb{C}_{W}(H)\right| \leq k$. Then there exist a subgroup $\tilde{A} \triangleleft A$ with $|A: \tilde{A}| \leq \alpha^{\prime}(k)$ and a subgroup $\tilde{G} \triangleleft G$ with $|G: \tilde{G}| \leq \beta^{\prime}(k,|A|)$ such that either $\tilde{A}$ or $\tilde{G}$ acts trivially on $W$. In particular, the commutator $[\tilde{A}, \tilde{G}]$ acts trivially on $W$.

Proof. We can, of course, assume that both $G$ and $A$ act faithfully and nontrivially on $W$ and, since $G=H^{G}$, it follows that $H$ also acts nontrivially. The goal is to suitably bound $|A|$ or $|G|$. There are several cases to consider according to whether $W$ is semisimple or elementary abelian, and according to whether the action of $G$ is primitive or not. We start with the two primitive situations.

Suppose first that $W$ is nonabelian simple. Since $\mathbb{C}_{W}(H)$ is a proper subgroup of $W$ of index $\leq k$, it follows that $|W| \leq k$ ! and thus $|G| \leq(k !)$ !. Next, let $W$ be elementary abelian and assume that $G$ acts in a primitive fashion on $W$. Since $A=[A, H]$ acts nontrivially, there exist elements $a, b \in A$ and $h \in H$ with $a=\left[b, h^{-1}\right]=h^{b} h^{-1}$ acting nontrivially on $W$. But

$$
\left|W: \mathbb{C}_{W}(a)\right| \leq\left|W: \mathbb{C}_{W}\left(h^{b}\right)\right|\left|W: \mathbb{C}_{W}(h)\right| \leq k^{2},
$$

so Lemma 3.2(ii) implies that $|W| \leq\left(k^{2}\right)^{2|A|}$ and therefore $|G| \leq|W|$ ! is bounded by a function of $k$ and $|A|$.

Now if $W$ is semisimple but not simple, then it follows from Lemma 2.9(ii) and irreducibility that $G$ permutes the simple factors of $W$ in a transitive manner. Similarly, if $W$ is an elementary abelian $G$-module which is not primitive, then $W$ is a direct product of isomorphic subgroups which are also permuted transitively by $G$. We consider both of these cases simultaneously.

Specifically, let us write $W=\prod_{i=1}^{t} W_{i}$ where $G$ permutes the factors $W_{i}$ in a transitive fashion and where $t>1$. Furthermore, assume that this situation is chosen with permutation degree $t>1$ minimal, and note that the latter minimality implies that $G$ acts primitively on $\Omega=\left\{W_{1}, W_{2}, \ldots, W_{t}\right\}$ since otherwise the factors $W_{i}$ could be combined into larger groups corresponding to nontrivial blocks of imprimitivity. Now $G=H^{G}$, so $H$ must also act nontrivially as a permutation group on $\Omega$. Consequently, by Lemma 3.3 and its notation, we conclude that $\left|W_{\mathcal{O}}\right| \leq k^{2}$ for all $H$-orbits $\mathcal{O}$, that $H$ centralizes all but at most $k$ of these $W_{\mathcal{O}}$, and that $\left|W_{i}\right| \leq k$.

We now consider the possible permutation actions of $A$ on $\Omega$. Suppose first that $A$ stabilizes all $W_{i}$ s. Then $A$ stabilizes each $W_{\mathcal{O}}$ and hence $\langle A, H\rangle$ acts on these groups. But note that $[A, H]=A$ and that $H$ can act nontrivially on at most $k$ of the $W_{\mathcal{O}}$. Thus $A$ can act nontrivially on at most $k$ of these $W_{\mathcal{O}}$ and, since $A$ acts faithfully on $W=\prod W_{\mathcal{O}}$, we conclude that $|A| \leq\left(\left(k^{2}\right) !\right)^{k}$.

On the other hand, suppose $A$ acts nontrivially on $\Omega$. Then using $[A, H]=A$, it follows that there exist elements $a, b \in A$ and $h \in H$ with $a=\left[b, h^{-1}\right]=h^{b} h^{-1}$ 
acting nontrivially on $\Omega$. Furthermore, since $h$ moves at most $k^{2}$ points of $\Omega$ by Lemma 3.3(iii), it follows that $a$ moves at most $2 k^{2}$ points. Thus $t=|\Omega| \leq 4 k^{2}|A|$ by Lemma 3.1(ii), so $|W|=\left|W_{1}\right|^{t} \leq k^{4 k^{2}|A|}$ and therefore $|G| \leq|W|$ ! is bounded by a function of $k$ and $|A|$.

Again, we allow for the possibility of infinite modules.

Lemma 3.5. Let $W \triangleleft V$ be possibly infinite groups and let $H$ act on $V$ and stabilize $W$. If $\left|V: \mathbb{C}_{V}(H)\right|=k<\infty$, then

i. $\left|W: \mathbb{C}_{W}(H)\right| \leq k$ with strict inequality if $H$ acts nontrivially on $V / W$, and

ii. $\left|V / W: \mathbb{C}_{V / W}(H)\right| \leq k$ with strict inequality if $H$ acts nontrivially on $W$.

Proof. Write $C=\mathbb{C}_{V}(H)$. Since $\mathbb{C}_{W}(H)=C \cap W$, we have

$$
\left|W: \mathbb{C}_{W}(H)\right|=|W: C \cap W|=|C W: C| \leq|V: C|=k .
$$

Furthermore, if equality occurs here then $V=C W$, so $H$ acts trivially on $V / W$.

Similarly, since $C W / W \subseteq \mathbb{C}_{V / W}(H)$, we have

$$
\left|V / W: \mathbb{C}_{V / W}(H)\right| \leq|V / W: C W / W|=|V: C W| \leq|V: C|=k .
$$

In addition, if equality occurs here then $C W=C$, so $W \subseteq C$ and therefore $H$ acts trivially on $W$.

Finally, we prove

Lemma 3.6. There exist two integer valued functions $\alpha$ and $\beta$ with the following property: Let $A$ and $H$ be subgroups of $G$ with $A \triangleleft \triangleleft G, G=H^{G}$ and $[A, H]=A$. Suppose $G$ acts on the finite group $W$ and that $\left|W: \mathbb{C}_{W}(H)\right| \leq k$. Then there exist a subgroup $\tilde{A} \triangleleft A$ with $|A: \tilde{A}| \leq \alpha(k)$ and a subgroup $\tilde{G} \triangleleft G$ with $|G: \tilde{G}| \leq \beta(k,|A|)$ such that the commutator group $[\tilde{A}, \tilde{G}]$ is unipotent on $W$.

Proof. Let $1=W_{0} \triangleleft W_{1} \triangleleft \cdots \triangleleft W_{n}=W$ be a subnormal series consisting of distinct $G$-stable subgroups of $W$ and chosen so that its length $n$ is maximal. Then it is clear that $G$ acts irreducibly on each $\bar{W}_{i}=W_{i} / W_{i-1}$. Furthermore, it follows from the preceding lemma and induction that $\left|\bar{W}_{i}: \mathbb{C}_{\bar{W}_{i}}(H)\right| \leq k$ and that $H$ can act nontrivially on at most $k$ of the $\bar{W}_{i}$. Thus $G=H^{G}$ can act nontrivially on at most $k$ of these quotients.

If $G$ acts nontrivially on $\bar{W}_{i}$, let $\tilde{A}_{i}$ and $\tilde{G}_{i}$ be the normal subgroups of $A$ and of $G$, respectively, given by Lemma 3.4. Thus $\left|A: \tilde{A}_{i}\right| \leq \alpha^{\prime}(k),\left|G: \tilde{G}_{i}\right| \leq \beta^{\prime}(k,|A|)$, and $\left[\tilde{A}_{i}, \tilde{G}_{i}\right]$ acts trivially on $\bar{W}_{i}$. On the other hand, if $G$ acts trivially on $\bar{W}_{i}$, set $\tilde{A}_{i}=A$ and $\tilde{G}_{i}=G$. Since $G$ can act nontrivially on at most $k$ of the quotients, it follows that $\tilde{A}=\bigcap_{i} \tilde{A}_{i}$ and $\tilde{G}=\bigcap_{i} \tilde{G}_{i}$ are normal subgroups of $A$ and of $G$, respectively, with $|A: \tilde{A}| \leq \alpha^{\prime}(k)^{k}=\alpha(k)$ and $|G: \tilde{G}| \leq \beta^{\prime}(k,|A|)^{k}=\beta(k,|A|)$. Furthermore, $[\tilde{A}, \tilde{G}] \subseteq\left[\tilde{A}_{i}, \tilde{G}_{i}\right]$ acts trivially on each $\bar{W}_{i}$, so the commutator group $[\tilde{A}, \tilde{G}]$ is unipotent on $W$.

\section{Local Subnormality}

Now let $G$ be a locally finite group. Recall that a finite subgroup $A$ of $G$ is said to be locally subnormal, written $A \operatorname{lsn} G$, if $A \triangleleft \triangleleft L$ for all finite subgroups $L$ of $G$ with $A \subseteq L \subseteq G$. Since this subnormality condition occurs locally, that is in finite subgroups of $G$, a number of results from the preceding sections carry over 
immediately to this new context. For example, the local analog of Lemma 2.1(i) asserts that if $A, B$ lsn $G$, then $A \cap B$ and $\langle A, B\rangle$ are also locally subnormal in $G$.

We will be concerned with the characteristic subgroup of $G$ given by

$$
\mathbb{S}(G)=\langle A \mid A \operatorname{lsn} G\rangle
$$

and with certain subgroups of $\mathbb{S}(G)$. For example, if $\pi$ is any set of primes, then we let $\mathbb{S}^{\pi}(G)$ denote the subgroup of $\mathbb{S}(G)$ generated by its $\pi$-elements. This notation differs somewhat from our earlier papers, but it seems more natural for our purposes. Of course, $\mathbb{S}^{p}(G)$ is the subgroup of $\mathbb{S}(G)$ generated by its $p$-elements.

As usual, a local system $\mathcal{L}$ for $G$ is a family of subgroups such that any finite subgroup $H$ of $G$ is contained in some $L \in \mathcal{L}$. It is easy to see that $\mathcal{L}$ is a local system if and only if (i) $G=\bigcup_{L \in \mathcal{L}} L$, and (ii) for all $L_{1}, L_{2} \in \mathcal{L}$ there exists $L \in \mathcal{L}$ with $L \supseteq\left\langle L_{1}, L_{2}\right\rangle$. With this notation, we have

Lemma 4.1. Let $G$ be a locally finite group.

i. The set of locally subnormal subgroups of $G$ is a local system for $\mathbb{S}(G)$.

ii. The set of locally subnormal subgroups $A$ of $G$ with $A=A^{\pi}$ is a local system for $\mathbb{S}^{\pi}(G)$. In particular,

$$
\left.\mathbb{S}^{\pi}(G)=\langle A| A \operatorname{lsn} G \text { and } A=A^{\pi}\right\rangle .
$$

iii. If $N$ is a subgroup of $G$, then $\mathbb{S}(G) \cap N \subseteq \mathbb{S}(N)$ with equality if $N \triangleleft \triangleleft G$.

iv. If $G$ is an infinite simple group, then $\mathbb{S}(G)=1$.

Proof. (i) This is an immediate consequence of the fact that if $A, B \operatorname{lsn} G$, then $\langle A, B\rangle \operatorname{lsn} G$.

(ii) If $H$ is a finite subgroup of $\mathbb{S}^{\pi}(G)$, then $H$ is contained in a finite subgroup $L$ of $\mathbb{S}^{\pi}(G)$ with $L=L^{\pi}$. Furthermore, by the above, $L$ is contained in a locally subnormal subgroup $B$ of $G$. Thus $H \subseteq L=L^{\pi} \subseteq B^{\pi}$, and it is clear that $A=B^{\pi}$ satisfies $A$ lsn $G$ and $A=A^{\pi}$.

(iii) If $A \operatorname{lsn} G$, then it is easy to see that $(A \cap N) \operatorname{lsn} N$ and, in view of (i), this implies that $\mathbb{S}(G) \cap N \subseteq \mathbb{S}(N)$. On the other hand, if $N \triangleleft \triangleleft G$ and if $B$ lsn $N$, then transitivity of subnormality implies that $B \operatorname{lsn} G$. As a consequence, we conclude that $\mathbb{S}(N) \subseteq \mathbb{S}(G)$, as required.

(iv) Suppose by way of contradiction that $A \neq 1$ is a locally subnormal subgroup of $G$, and let $B$ be a finite subgroup of $G$ properly containing $A$. Since $G$ is infinite simple, [KWe, Proposition 4.6] implies that $G$ has finite subgroups $M \triangleleft L$ with $B \subseteq L, L / M$ simple, and $B \cap M=1$. Now $A \triangleleft \triangleleft L$ and $A \not \subset M$, so it follows that $1 \neq A M / M \triangleleft \triangleleft L / M$ and hence $A M=L \supseteq B$. Thus $B=A(M \cap B)=A$, and this contradicts the choice of $B$.

Next we define the Fitting subgroup of $G$ by

$$
\mathbb{F}(G)=\langle A| A \operatorname{lsn} G \text { and } A \text { is nilpotent }\rangle .
$$

Basic properties of this subgroup are as follows.

Lemma 4.2. Let $G$ be a locally finite group.

i. The set of locally subnormal nilpotent subgroups of $G$ is a local system for the Fitting subgroup $\mathbb{F}(G)$.

ii. $\mathbb{F}(G)=\prod_{q} \mathbb{O}_{q}(G)$, where $\prod_{q}$ indicates a direct product and where $\mathbb{O}_{q}(G)$ is the unique largest normal q-subgroup of $G$.

iii. If $N \subseteq G$, then $\mathbb{F}(G) \cap N \subseteq \mathbb{F}(N)$ with equality if $N \triangleleft \triangleleft G$. 
Proof. Parts (i) and (iii) follow, as in the preceding lemma, using the local version of Lemma 2.2(ii). For part (ii), note that if $A$ is a locally subnormal nilpotent subgroup of $G$, then $A=\prod_{q} \mathbb{O}_{q}(A)$ and $\mathbb{O}_{q}(A) \subseteq \mathbb{O}_{q}(G)$ by the local version of Lemma 2.2(i). Thus $\mathbb{F}(G) \subseteq \prod_{q} \mathbb{O}_{q}(G)$. Conversely, since $\mathbb{O}_{q}(G)$ is a locally nilpotent normal subgroup of $G$, every finite subgroup of $\mathbb{O}_{q}(G)$ is a locally subnormal nilpotent subgroup of $G$. Thus $\mathbb{O}_{q}(G) \subseteq \mathbb{F}(G)$ and the reverse inclusion is proved.

For a number of reasons, the generalized Fitting subgroup of $G$, given by

$$
\left.\mathbb{F}^{*}(G)=\langle A| A \operatorname{lsn} G \text { and } A \text { is nilpotent or quasi-simple }\right\rangle,
$$

is the key subgroup of interest here. To start with, there is a local analog of Fitting's lemma based on this group and using the following local version of unipotent action. Let $U$ be a finite group acting as automorphisms on the locally finite group $V$. Then we say that $U$ is locally unipotent on $V$ if, for each $U$-stable finite subgroup $W$ of $V$, we have

$$
[W, U, U, \ldots, U]=[W, U]_{n}=1
$$

for some integer $n=n(W)$. In other words, this occurs precisely when $U$ is unipotent in its action on each such $W$.

Lemma 4.3. Let $\mathbb{F}^{*}(G)$ be the generalized Fitting subgroup of $G$

i. If $U \operatorname{lsn} G$, then $U$ is locally unipotent on $\mathbb{F}^{*}(G)$ if and only if $U \subseteq \mathbb{F}(G)$.

ii. $\mathbb{C}_{\mathbb{S}(G)}\left(\mathbb{F}^{*}(G)\right)=\mathbb{Z}(\mathbb{F}(G))$.

iii. If $N \triangleleft \triangleleft G$, then $\mathbb{F}^{*}(G) \cap N=\mathbb{F}^{*}(N)$.

Proof. (i) Let $U \operatorname{lsn} G$ be locally unipotent in its action on $\mathbb{F}^{*}(G)$. We show that $U \subseteq \mathbb{F}(G)$ by induction on $|U|$, the result being trivial if $U=1$. Now suppose that $U \neq 1$ and let $M$ be a maximal normal subgroup of $U$. Then $M \operatorname{lsn} G$ and $M$ is locally unipotent on $\mathbb{F}^{*}(G)$, so $M \subseteq \mathbb{F}(G)$ by induction. In particular, $[M, U]_{n}=1$ for some integer $n$, and Lemma 2.6 implies that either $U$ is nilpotent or $U=M U^{(\infty)}$ with $M$ nilpotent and with $U^{(\infty)}$ quasi-simple. In either case, since $U^{(\infty)} \triangleleft U$, it follows that $U \subseteq \mathbb{F}^{*}(G)$. But then $U$ is unipotent on $U$, so $U$ is nilpotent and $U \subseteq \mathbb{F}(G)$, as required.

Conversely, suppose $U \subseteq \mathbb{F}(G)$ and let $L$ be any finite subgroup of $G$ containing $U$. Then $U \triangleleft \triangleleft L$, so Lemma 2.5(i) implies that $[L, U]_{n} \subseteq U$ for some integer $n$. In addition, since $U$ is nilpotent, we have $[U, U]_{m}=1$ for some $m$ and therefore $[L, U]_{n+m} \subseteq[U, U]_{m}=1$. With this, it is clear that $U$ is locally unipotent in its action on $G$ and hence on $\mathbb{F}^{*}(G)$.

(ii) By Lemma 4.1, $C=\mathbb{C}_{\mathbb{S}(G)}\left(\mathbb{F}^{*}(G)\right)$ is generated by locally subnormal subgroups of $\mathbb{S}(G)$ and hence of $G$. Since each such subgroup is clearly locally unipotent on $\mathbb{F}^{*}(G)$, it follows from the above that $C \subseteq \mathbb{F}(G)$ and hence that $C \subseteq \mathbb{Z}(\mathbb{F}(G))$. Conversely, note that $\mathbb{Z}(\mathbb{F}(G))$ centralizes $\mathbb{F}(G)$ and that it also centralizes each quasi-simple locally subnormal subgroup of $G$ by the local version of Lemma 2.7(ii). Thus $\mathbb{Z}(\mathbb{F}(G))$ centralizes $\mathbb{F}^{*}(G)$ and this yields the reverse inclusion.

(iii) Since $N \triangleleft \triangleleft G$, any locally subnormal subgroup of $N$ is locally subnormal in $G$, and it follows that $\mathbb{F}^{*}(N) \subseteq \mathbb{F}^{*}(G) \cap N$. Conversely, note that any finite subgroup of $\mathbb{F}^{*}(G)$ is contained in a locally subnormal subgroup $A$ of $G$ generated by subnormal subgroups which are either nilpotent or quasi-simple. Thus, since $N \triangleleft \triangleleft G$, we have $(A \cap N) \triangleleft \triangleleft A$ and hence Lemma 2.8 implies that $A \cap N$ is also generated by subnormal subgroups which are either nilpotent or quasi-simple. Furthermore, 
since $(A \cap N)$ lsn $N$, the subnormal subgroups of $A \cap N$ are locally subnormal in $N$. Consequently, $A \cap N \subseteq \mathbb{F}^{*}(N)$ and we have the reverse inclusion, namely $\mathbb{F}^{*}(G) \cap N \subseteq \mathbb{F}^{*}(N)$.

Note that parts (i) and (ii) above show that $\mathbb{F}^{*}(G)$ is "large" in $\mathbb{S}(G)$. On the other hand, the next result shows that it is "small". For this, we need some additional notation.

If $H$ and $V$ are subgroups of $G$, then we define the almost or finitary centralizer of $V$ in $H$ by

$$
\mathbb{D}_{H}(V)=\left\{h \in H|| V: \mathbb{C}_{V}(h) \mid<\infty\right\},
$$

and it is easy to see that $\mathbb{D}_{H}(V)$ is a subgroup of $H$. Furthermore, if $H$ normalizes $V$, then $\mathbb{D}_{H}(V)$ is the normal subgroup of $H$ consisting of those elements which act in a finitary manner on $V$. Note that

$$
\mathbb{D}_{G}(G)=\Delta(G)=\left\{x \in G|| G: \mathbb{C}_{G}(x) \mid<\infty\right\},
$$

and this group is called the finite conjugate or f.c. center of $G$ since it consists of those elements belonging to finite conjugacy classes. We say that $G$ is an f.c. group if $G=\Delta(G)$ and, in the context of locally finite groups, this is equivalent to $G$ being locally normal. In other words, $G=\Delta(G)$ if and only if every finite subgroup of $G$ is contained in a finite normal subgroup (see [P5, Lemma 4.1.8]). In fact, if $G$ is an arbitrary periodic group, then $\Delta(G)$ is generated by, and hence is the join of, the finite normal subgroups of $G$.

Lemma 4.4. Let $\pi$ be a set of primes and let $G$ be a locally finite group with $\mathbb{O}_{\pi}(\mathbb{F}(G))$ finite. Then

$$
\mathbb{S}^{\pi}(G) \subseteq \mathbb{D}_{G}\left(\mathbb{F}^{*}(G)\right)
$$

In particular, if $\mathbb{F}^{*}(G) \subseteq \mathbb{S}^{\pi}(G)$, then $\mathbb{F}^{*}(G)$ is an f.c. group.

Proof. Let $N$ be the subgroup of $\mathbb{F}^{*}(G)$ generated by all locally subnormal subgroups $A$ such that $A$ is either a nilpotent $\pi^{\prime}$-group or a quasi-simple group. Then clearly $\mathbb{F}^{*}(G)=N \mathbb{O}_{\pi}(\mathbb{F}(G))$, so the hypothesis implies that $\left|\mathbb{F}^{*}(G): N\right|<\infty$. Now fix $B \operatorname{lsn} G$ with $B=B^{\pi}$ and let $A$ be a generator of $N$. If $A$ is nilpotent, then the local version of Lemma 2.10 implies that $A$ normalizes $B$, and if $A$ is quasisimple, then the local version of Lemma 2.7(ii) yields the same conclusion. Thus $N$ normalizes $B$ and, since $B$ is finite, we conclude that $B$ has a centralizer of finite index in $N$ and hence in $\mathbb{F}^{*}(G)$. In other words, $B \subseteq \mathbb{D}_{G}\left(\mathbb{F}^{*}(G)\right)$ and, since $\mathbb{S}^{\pi}(G)$ is generated by all such $B$, the result follows.

Let $H \subseteq X$ be finite groups. Since the set of subnormal subgroups of $X$ is closed under intersection, it follows that there is a unique smallest subnormal subgroup $S$ of $X$ which contains $H$. This is called the subnormal closure of $H$ in $X$, and we denote it by $S=H^{[X]}$. If $H^{S}$ is the normal closure of $H$ in $S$, then $H \subseteq H^{S} \triangleleft S \triangleleft \triangleleft X$, so the minimal nature of $S$ implies that $S=H^{S}$. In fact, $S$ is characterized by the two properties

i. $H \subseteq S \triangleleft \triangleleft X$, and

ii. $S=H^{S}$,

since (ii) implies that $H$ cannot be contained in a proper normal subgroup of $S$, and hence it is not in a proper subnormal subgroup of $S$. Note that, if $\bar{X}$ is a homomorphic image of $X$, and if $H$ and $S$ are as above, then $\bar{H} \subseteq \bar{S} \triangleleft \triangleleft \bar{X}$ and 
$\bar{S}=\bar{H}^{\bar{S}}$. Thus $\bar{S}$ is the subnormal closure of $\bar{H}$ in $\bar{X}$. In general, subnormal closures do not exist for arbitrary subgroups of infinite groups.

Now if $H \subseteq X \subseteq Y$ are all finite, then $H \subseteq H^{[Y]} \cap X \triangleleft \triangleleft X$. Thus the minimal nature of $H^{[X]}$ implies that $H^{[X]} \subseteq H^{[Y]} \cap X \subseteq H^{[Y]}$, and this inclusion allows us to define a local subnormal closure for finite subgroups of locally finite groups. Specifically, if $H$ is a finite subgroup of the locally finite group $G$, then we set

$$
H^{[G]}=\bigcup_{X} H^{[X]}
$$

where the union is over all finite subgroups $X$ of $G$ containing $H$. Note that, if $G$ is finite, then the inclusion $H^{[X]} \subseteq H^{[Y]}$ immediately implies that the two possible meanings for $H^{[G]}$ are, in fact, the same. Some basic properties are as follows.

Lemma 4.5. Let $H$ be a finite subgroup of $G$, and set $S=H^{[G]}$.

i. $S$ is a subgroup of $G$ containing $H$, and $S=H^{[S]}$ is the local subnormal closure of $H$ in $S$.

ii. If $\mathcal{L}$ is a local system for $G$, then $\left\{H^{[L]} \mid L \in \mathcal{L}, L \supseteq H\right\}$ is a local system for the subgroup $S$.

iii. If $A \operatorname{lsn} S$, then $A \operatorname{lsn} G$.

iv. $S=H^{S}$ is the normal closure of $H$ in $S$, and $\mathbb{N}_{G}(H) \subseteq \mathbb{N}_{G}(S)$.

v. If ${ }^{-}: G \rightarrow \bar{G}$ is an epimorphism, then $\bar{S}=\bar{H}^{[\bar{G}]}$.

Proof. (i) If $X$ and $Y$ are finite subgroups of $G$ containing $H$ and if $Z=\langle X, Y\rangle$, then $\left\langle H^{[X]}, H^{[Y]}\right\rangle \subseteq H^{[Z]} \subseteq H^{[G]}$. Thus $S=H^{[G]}$ is a subgroup of $G$ with the set of all such $H^{[X]}$ as a local system. Finally, if $L=H^{[X]} \subseteq S$, then we know that $H$ is contained in no proper subnormal subgroup of $L$. Thus $L=H^{[L]} \subseteq H^{[S]}$ and $H^{[S]} \supseteq \bigcup_{X} H^{[X]}=S$.

(ii) Let $X$ be a finite subgroup of $S$. Then (i) implies that $X \subseteq H^{[Y]}$ for some finite subgroup $Y$ of $G$ containing $H$. Furthermore, since $\mathcal{L}$ is a local system for $G$, there exists $L \in \mathcal{L}$ with $Y \subseteq L$. Thus $X \subseteq H^{[Y]} \subseteq H^{[L]} \subseteq H^{[G]}$, as required.

(iii) Let $A$ lsn $S$ and, by (i), let $X$ be a finite subgroup of $G$ containing $H$ with $A \subseteq H^{[X]}$. Now let $B$ be any finite subgroup of $G$ containing $X$. Then $A \subseteq H^{[X]} \subseteq H^{[B]} \subseteq H^{[G]}$, so $A \triangleleft \triangleleft H^{[B]}$ since $A \operatorname{lsn} S$. But $H^{[B]} \triangleleft \triangleleft B$, so we conclude that $A \triangleleft \triangleleft \bar{B}$, and hence $A \operatorname{lsn} G$.

(iv) The equality $S=H^{S}$ is clear since $H^{S}$ contains each $H^{[X]}$. For the remaining part, let $g \in \mathbb{N}_{G}(H)$ and let $Y$ be any finite subgroup of $G$ containing $g$ and $H$. Then $H \subseteq H^{[Y]}$ implies that $H=g^{-1} H g \subseteq g^{-1} H^{[Y]} g$. But the latter group is also subnormal in $Y$, so $g^{-1} H^{[Y]} g=H^{[Y]}$ and it follows that $g$ normalizes $S=H^{[G]}=\bigcup_{Y} H^{[Y]}$.

(v) If $X$ is a finite subgroup of $G$ containing $H$, then $\bar{X}$ is a finite subgroup of $\bar{G}$ containing $\bar{H}$. Conversely, if $Y$ is a finite subgroup of $\bar{G}$ containing $\bar{H}$, then there exists a finite subgroup $X$ of $G$ containing $H$ with $\bar{X}=Y$. Furthermore, as we observed above, $\bar{H}^{[\bar{X}]}$ is the image of $H^{[X]}$ under the map ${ }^{-}: X \rightarrow \bar{X}$. Thus, since $S=H^{[G]}=\bigcup_{X} H^{[X]}$ and $\bar{H}^{[\bar{G}]}=\bigcup_{\bar{X}} \bar{H}^{[\bar{X}]}$, the result follows.

We remark that part (iii) above allows us to reduce semiprimitivity considerations to certain local subnormal closures. However, it is the condition $S=H^{S}$ in part (iv) which really turns out to be crucial. We close this section with

Lemma 4.6. Let $H$ be a finite subgroup of $G$ and set $S=H^{[G]}$. Then

i. $\mathbb{S}(G) \cap S=\mathbb{S}(S)$, 
ii. $\mathbb{F}(G) \cap S=\mathbb{F}(S)$, and

iii. $\mathbb{F}^{*}(G) \cap S=\mathbb{F}^{*}(S)$.

Proof. By Lemma 4.5(iii) we have $\mathbb{S}(S) \subseteq \mathbb{S}(G), \mathbb{F}(S) \subseteq \mathbb{F}(G)$ and $\mathbb{F}^{*}(S) \subseteq \mathbb{F}^{*}(G)$. Thus parts (i) and (ii) of this lemma follow immediately from Lemmas 4.1(iii) and 4.2(iii). It remains to show that $\mathbb{F}^{*}(G) \cap S \subseteq \mathbb{F}^{*}(S)$.

To this end, let $A \operatorname{lsn} G$ be generated by subnormal subgroups which are either nilpotent or quasi-simple and let $L$ be any finite subgroup of $S=H^{[G]}$ containing $A \cap S$. By Lemma 4.5(i), there exists a finite subgroup $X$ of $G$ containing $H$ with $L \subseteq H^{[X]}$, and we set $Y=\langle A, X\rangle$. Then $B=H^{[Y]} \triangleleft \triangleleft Y$ and

$$
A \cap S \subseteq L \subseteq H^{[X]} \subseteq H^{[Y]}=B \subseteq S .
$$

Now observe that both $A$ and $B$ are subnormal in $Y$ since $A$ lsn $G$. Thus, since $A \cap S=A \cap B$, we see that $A \cap S \triangleleft \triangleleft Y$. In particular, $A \cap S \triangleleft \triangleleft L$ and we conclude that $A \cap S$ lsn $S$. Furthermore $A \cap S \triangleleft \triangleleft A$, so Lemma 2.8 implies that $A \cap S$ is generated by subnormal subgroups which are either nilpotent or quasi-simple. Indeed, since $A \cap S \operatorname{lsn} S$, these subnormal subgroups are all locally subnormal in $S$ and hence contained in $\mathbb{F}^{*}(S)$. In other words, $A \cap S \subseteq \mathbb{F}^{*}(S)$ and the lemma is proved.

\section{Finitary Linear Groups}

Next, we turn our attention to locally finite, finitary groups. To start with, we say that $G$ acts in a finitary manner on the group $V$ if $\left|V: \mathbb{C}_{V}(x)\right|<\infty$ for all $x \in G$. Furthermore, $G$ acts in a strongly finitary manner if the action is finitary and if all $G$-stable subgroups of $V$ are normal in $V$. In particular, both of these concepts include the usual notion of a finitary action of a group $G$ on a vector space $V$ over a finite field. Notice that we do not assume, at this point, that $G$ acts faithfully on $V$. Note further that if $G$ is strongly finitary on $V$ and if $W$ is a $G$-stable subgroup of $V$, then $G$ acts in a strongly finitary manner on both $W$ and $V / W$. Of course, $G$ acts irreducibly on $V$ if and only if $V$ has no proper $G$-stable normal subgroup.

Lemma 5.1. Let $G$ act in a strongly finitary manner on the group $V$, and assume that $G=H^{G}$ is the normal closure of some finite subgroup $H$. Then $V$ has a finite chain

$$
1=V_{0} \subseteq V_{1} \subseteq \cdots \subseteq V_{n}=V
$$

of $G$-stable normal subgroups such that, for each $i$, either $G$ acts irreducibly on $\bar{V}_{i}=V_{i} / V_{i-1}$ or it acts trivially on this quotient.

Proof. Since $G$ acts in a strongly finitary manner on $V$ and since $H$ is a finite subgroup of $G$, it follows that $\left|V: \mathbb{C}_{V}(H)\right|=k<\infty$, and we proceed by induction on $k$. Of course if $k=1$, then $H$ centralizes $V$ and hence $G=H^{G}$ also centralizes $V$. Now suppose that $k>1$ and that the result holds for all such situations with parameter less than $k$.

Let $X_{1}=\mathbb{C}_{V}(G)$, so that $X_{1}$ is the largest $G$-stable normal subgroup of $V$ on which $G$ acts trivially. Furthermore, let $X_{2} / X_{1}$ be the centralizer of $G$ in $V / X_{1}$, and note that $X_{2}$ is also $G$-stable. If $X_{2}$ is strictly larger than $X_{1}$, then $G$, and hence also $H$, acts nontrivially on this group. Consequently, Lemma 3.5(ii) implies that $\left|V / X_{2}: \mathbb{C}_{V / X_{2}}(H)\right|<k$. Thus, by induction, $V / X_{2}$ has an appropriate $G$-stable series, and therefore the same is clearly true for $V$. In other words, it now suffices 
to assume that $X_{2}=X_{1}$, and since $\left|V / X_{1}: \mathbb{C}_{V / X_{1}}(H)\right| \leq k$, we can replace $V$ by $V / X_{1}$ and assume that $\mathbb{C}_{V}(G)=1$.

Similarly, observe that $Y_{1}=[V, G]$ is a $G$-stable subgroup of $V$ and that $G$ acts trivially on $V / Y_{1}$. Furthermore, if $Y_{2}=\left[Y_{1}, G\right]$, then $G$ stabilizes the chain $1 \subseteq Y_{1} / Y_{2} \subseteq V / Y_{2}$ and acts trivially on each factor. Again, if $Y_{1}$ is strictly larger than $Y_{2}$, then $G$, and hence also $H$, acts nontrivially on $V / Y_{2}$, so Lemma 3.5(i) implies that $\left|Y_{2}: \mathbb{C}_{Y_{2}}(H)\right|<k$. Thus, by induction, $Y_{2}$ has an appropriate $G$-stable series, and therefore the same is clearly true for $V$. Consequently, it suffices to assume that $Y_{1}=Y_{2}$, and we can now replace $V$ by $Y_{1}$ and suppose that $[V, G]=V$. In particular, $G$ acts nontrivially on every nonidentity $G$-stable factor group of $V$.

Now if $G$ acts irreducibly on $V$, then we are done. Thus, we may suppose that $W$ is a nontrivial $G$-stable subgroup of $V$. By the comments of the preceding two paragraphs, we know that $G$, and hence also $H$, acts nontrivially on both $W$ and $V / W$. Thus Lemma 3.5 yields $\left|W: \mathbb{C}_{W}(H)\right|<k$ and $\left|V / W: \mathbb{C}_{V / W}(H)\right|<k$, so induction applies to the action of $G$ on $W$ and on $V / W$. Consequently, both $W$ and $V / W$ have appropriate $G$-stable normal series, and by putting these together we obtain such a series for $V$.

For convenience, we record the following elementary observation.

Lemma 5.2. Let $G$ act in a finitary manner on $V$, and let $H$ be a finite normal subgroup of $G$.

i. $\mathbb{C}_{V}(H)$ is a $G$-stable subgroup of $V$ of finite index, and $[V, H]$ is a finite $G$-stable subgroup of $V$.

ii. If $G$ acts irreducibly on $V$ and $V$ is infinite, then $H$ acts trivially on $V$. In particular, $\Delta(G)$ acts trivially on $V$.

Proof. Part (i) is obvious since $V$ is locally finite. For (ii), note that $G$ stabilizes $\mathbb{C}_{V}(H)$, a subgroup of $V$ of finite index, and hence $G$ stabilizes $N=$ core $_{V} \mathbb{C}_{V}(H)$, the largest normal subgroup of $V$ contained in $\mathbb{C}_{V}(H)$. But $G$ acts irreducibly on the infinite group $V$, and $|V: N|<\infty$. Consequently, $V=N \subseteq \mathbb{C}_{V}(H)$, and $H$ acts trivially on $V$.

We can now prove the version of [P4, Theorem 4.1] we require.

Proposition 5.3. Let $H$ be a finite subgroup of the locally finite group $G$ and suppose that $G=\mathbb{S}(G) H$. If $\left|\mathbb{F}^{*}(G): \mathbb{C}_{\mathbb{F}^{*}(G)}(H)\right|<\infty$, then $G=\mathbb{S}(G)$.

Proof. It clearly suffices to prove that $H \subseteq \mathbb{S}(G)$ and, for this, we proceed in a series of steps.

Step 1. We can assume that $G=H^{[G]}$ is the local subnormal closure of $H$. In particular, $G=H^{G}=\mathbb{D}_{G}\left(\mathbb{F}^{*}(G)\right)$ and $\mathbb{F}^{*}(G)$ is an f.c. group.

Proof. Let $S=H^{[G]}$ be the local subnormal closure of $H$ in $G$ and observe that $H \subseteq S \subseteq G=\mathbb{S}(G) H$. Thus $S=(S \cap \mathbb{S}(G)) H=\mathbb{S}(S) H$ by Lemma 4.6(i). Furthermore, $\mathbb{F}^{*}(S) \subseteq \mathbb{F}^{*}(G)$, so $\left|\mathbb{F}^{*}(S): \mathbb{C}_{\mathbb{F}^{*}(S)}(H)\right| \leq\left|\mathbb{F}^{*}(G): \mathbb{C}_{\mathbb{F}^{*}(G)}(H)\right|<\infty$. In particular, $S$ satisfies the same hypotheses as $G$, and since $\mathbb{S}(S) \subseteq \mathbb{S}(G)$, it suffices to prove that $H \subseteq \mathbb{S}(S)$. Finally, note that $S=H^{[S]}=H^{S}$ by Lemma 4.5(i)(iv), and $S=H^{S} \subseteq \mathbb{D}_{S}\left(\mathbb{F}^{*}(S)\right)$ since $H \subseteq \mathbb{D}_{S}\left(\mathbb{F}^{*}(S)\right) \triangleleft S$. In other words, if we now replace $G$ by $S$, then $G$ has all the appropriate properties. 
Write $F=\mathbb{F}(G)$ and $V=\mathbb{F}^{*}(G)$ so that, by hypothesis, $\left|V: \mathbb{C}_{V}(H)\right|=k<\infty$. Furthermore, let

$$
\mathcal{E}=\{A \mid A \operatorname{lsn} G \text { and }[A, H]=A\}
$$

and define $E=\langle A \mid A \in \mathcal{E}\rangle$. Then $E \subseteq \mathbb{S}(G)$ and, as it turns out, our goal is to prove that $E$ is finite.

Step 2. If $\bar{G}=G / F$, then $|\bar{E}: \bar{E} \cap \Delta(\bar{G})|<\infty$.

Proof. Note that $\mathcal{E}$ is a local system for $E$ and that, by Lemma 4.5,

$$
\mathcal{L}=\left\{L \mid L \text { is finite, } L \supseteq H \text {, and } L=H^{L}\right\}
$$

is a local system for $G=H^{[G]}$. Let $A \in \mathcal{E}$ and let $L \in \mathcal{L}$ with $L \supseteq A$. Since $\left|V: \mathbb{C}_{V}(L)\right|<\infty$, it follows that the commutator group $W=[V, L]$ is finite and $L$-stable. Furthermore, the action of $L$ on $W$ satisfies the hypotheses of Lemma 3.6. Thus, the latter result implies that there exist subgroups $\widetilde{A}_{L} \triangleleft A$ with $\left|A: \tilde{A}_{L}\right| \leq$ $\alpha(k)$ and $\tilde{L} \triangleleft L$ with $|L: \tilde{L}| \leq \beta(k,|A|)$, such that the commutator group $\left[\tilde{A}_{L}, \tilde{L}\right]$ is unipotent on $W$. Indeed, since $W=[V, L]$, we see that $L$ acts trivially on $V / W$, and therefore $\left[\tilde{A}_{L}, \tilde{L}\right]$ is unipotent on $V$.

Note that $\left[\tilde{A}_{L}, \tilde{L}\right] \triangleleft \tilde{L} \triangleleft L$ and that $\left[\tilde{A}_{L}, \tilde{L}\right] \subseteq A^{L}$. Thus, since $A^{L}$ lsn $G$ and $A^{L} \subseteq L$, it follows that $\left[\tilde{A}_{L}, \tilde{L}\right] \operatorname{lsn} G$, and we conclude from Lemma $4.3(\mathrm{i})$ that $\left[\tilde{A}_{L}, \tilde{L}\right] \subseteq F$. In other words, $\tilde{A}_{L}$ and $\tilde{L}$ commute modulo $F$, so the centralizer of $\tilde{A}_{L} F / F$ in $L F / F$ has index $\leq \beta(k,|A|)$. Of course, $\tilde{A}_{L}$ depends upon $L$, but since $A$ has only finitely many subgroups and since $\mathcal{L}$ is a local system for $G$, it follows easily from the above that there exists $\tilde{A} \triangleleft A$ with $|A: \tilde{A}| \leq \alpha(k)$ and such that $\tilde{A} F / F$ has centralizer of index $\leq \beta(k,|A|)$ in $\bar{G}=G / F$.

Thus $\tilde{A} F / F \subseteq \Delta(\bar{G})$ and hence $|\bar{A}: \bar{A} \cap \Delta(\bar{G})| \leq \alpha(k)$. But $\mathcal{E}$ is a local system for $E$, so it now follows easily that $|\bar{E}: \bar{E} \cap \Delta(\bar{G})| \leq \alpha(k)$ and, in particular, this index is finite.

Step 3. $|E: E \cap F|<\infty$.

Proof. Since $G=\mathbb{D}_{G}(V)$ and $G \supseteq V$, it follows that $G$ is strongly finitary in its conjugation action on $V$. Furthermore, since $G=H^{G}$, we conclude from Lemma 5.1 that $V$ has a finite chain

$$
1=V_{0} \subseteq V_{1} \subseteq \cdots \subseteq V_{n}=V
$$

of $G$-stable normal subgroups such that, for each $i$, either $G$ acts irreducibly on $\bar{V}_{i}=V_{i} / V_{i-1}$ or it acts trivially on this quotient. We first observe that $F$ is trivial on each $\bar{V}_{i}$.

For this, we can certainly assume that $G$ acts irreducibly on $\bar{V}_{i}$. Furthermore, since $V$ is an f.c. group, there exists a finite normal subgroup $N_{i}$ of $V$, contained in $V_{i}$, with $1 \neq \bar{N}_{i}=N_{i} V_{i-1} / V_{i-1} \subseteq V_{i} / V_{i-1}$. If $D_{i}=\mathbb{C}_{F}\left(N_{i}\right)$, then $D_{i} \triangleleft F$ and $\left|F: D_{i}\right|<\infty$. Thus there exists $A_{i} \operatorname{lsn} F$ with $F=D_{i} A_{i}$ and, by Lemma $4.3(\mathrm{i})$, the action of $A_{i}$ on $V$ is locally unipotent. Thus $A_{i}$ is unipotent on $N_{i}$, so it follows that $F$ is unipotent on $\bar{N}_{i}$ and hence clearly $\mathbb{C}_{\bar{N}_{i}}(F) \neq 1$. Consequently, $\mathbb{C}_{\bar{V}_{i}}(F) \neq 1$ and, since the latter is a $G$-stable subgroup of $\bar{V}_{i}$, we must have $\mathbb{C}_{\bar{V}_{i}}(F)=\bar{V}_{i}$ and therefore $F$ does indeed act trivially on this module.

In other words, $G / F$ acts on each $\bar{V}_{i}$. Furthermore, it follows from the preceding lemma that $\Delta(G / F)$ acts trivially on $\bar{V}_{i}$ if either $G$ acts trivially on $\bar{V}_{i}$ or $G$ acts irreducibly on $\bar{V}_{i}$ with $\bar{V}_{i}$ infinite. Consequently, Step 2 implies that $E$ has a normal 
subgroup $E_{0}$ of finite index such that $E_{0} F / F$ acts trivially on each $\bar{V}_{i}$. Thus $E_{0}$ is unipotent on $V$, and since $E_{0}$ is generated by locally subnormal subgroups of $G$, it follows that $E_{0} \subseteq F$ and hence that $|E: E \cap F|<\infty$.

Step 4. $E$ is finite and $E H \operatorname{lsn} G$. Thus $H \subseteq \mathbb{S}(G)$, as required.

Proof. Since $H$ centralizes a subgroup of $F$ of finite index and since $|E: E \cap F|<$ $\infty$, it follows that $H$ centralizes a subgroup of $E$ of finite index. In particular, $H \subseteq \Delta(E H)$ and, since $H$ is finite, this implies that $[E, H]$ is finite. But certainly $E=[E, H]$ and therefore we conclude that $E$ is indeed finite. The goal now is to show that $E H \operatorname{lsn} G$. To this end, let $L$ be any finite subgroup of $G$ containing $E H$. Since $G=\mathbb{S}(G) H$, we have $L=(L \cap \mathbb{S}(G)) H$ and thus $L \subseteq A H$ for some $A$ lsn $G$ with $A^{H}=A$ and $A \supseteq E$.

Now define the groups $A_{i}$ inductively by $A_{0}=A$ and $A_{i+1}=\left[A_{i}, H\right]$. By Lemma 2.3(i) we have $A_{i+1} \triangleleft A_{i}$, so each $A_{i}$ is an $H$-stable locally subnormal subgroup of $G$. Furthermore, $A_{0} \supseteq E$ and if $A_{i} \supseteq E$, then $A_{i+1}=\left[A_{i}, H\right] \supseteq$ $[E, H]=E$. Thus we see that $A_{i} \supseteq E$ for all $i$. Now $A_{0}$ is finite, so the descending series $A_{0} \supseteq A_{1} \supseteq A_{2} \supseteq \cdots$ must terminate at some $A_{n}$. Thus $A_{n}$ lsn $G$ and $A_{n}=A_{n+1}=\left[A_{n}, H\right]$, so $A_{n} \in \mathcal{E}$ and $A_{n} \subseteq E$. Consequently, $A_{n}=E$ and we have the descending series

$$
A H=A_{0} H \supseteq A_{1} H \supseteq A_{2} H \supseteq \cdots \supseteq A_{n} H=E H .
$$

Finally, by definition of $A_{i+1}$, it follows that $A_{i+1} H \triangleleft A_{i} H$. Thus $E H \triangleleft \triangleleft A H$ and therefore $E H \triangleleft \triangleleft L$ since $E H \subseteq L \subseteq A H$. Indeed, since $L$ is arbitrary, we have $E H \operatorname{lsn} G$, so $H \subseteq E H \subseteq \mathbb{S}(G)$ and the proposition is proved.

For convenience, we say that a locally finite group $V$ is semisimple if it is a (weak) direct product of finite nonabelian simple groups. As usual, any normal subgroup of $V$ is a partial direct product of these factors and, consequently, the minimal normal subgroups of $V$ are precisely the nonabelian simple factors.

We now take a closer look at the possible finitary irreducible representations of $G$ on a not necessarily abelian group $V$. The following lemma serves to define the Primitive and Imprimitive Cases. It uses wonderful results from [Ph2] and [W2].

Proposition 5.4. Let $G$ act irreducibly and in a finitary manner on the infinite locally finite f.c. group $V$. If $G=H^{G}$ for some finite subgroup $H$ of $G$ and if $N$ is the kernel of the action of $G$ on $V$, then we have the following two possibilities.

i. (The Primitive Case) $V$ is an elementary abelian q-group for some prime $q$ and $V$ is a primitive $\operatorname{GF}(q)[G]$-module. In particular, if $G^{(6)}$ denotes the sixth derived subgroup of $G$, then $G^{(6)} N / N$ is an infinite simple, finitary linear group.

ii. (The Imprimitive Case) $V=\prod_{i \in \mathcal{I}} V_{i}$ is a (weak) direct product of finite normal subgroups which are permuted by $G$. If $L$ is the kernel of the permutation action of $G$ on the collection of $V_{i} s$, then $G / L=\mathrm{FSym}_{\mathcal{I}}$ or $\mathrm{FAlt}_{\mathcal{I}}$. Furthermore, $L / N$ is isomorphic to a subgroup of the (weak) direct product $\prod_{i \in \mathcal{I}} \operatorname{Aut}\left(V_{i}\right)$ and therefore $L / N$ is an f.c. group.

Proof. Since $V$ is an f.c. group, it has a finite minimal normal subgroup $W$ which is either an elementary abelian $q$-group for some prime $q$ or a direct product of nonabelian simple groups. Since any two distinct minimal normal subgroups of $V$ commute and since $V=W^{G}$, it follows that either $V$ is an elementary abelian $q$-group or it is semisimple. 
Suppose first that $V$ is an elementary abelian $q$-group, so that $\bar{G}=G / N$ is an irreducible finitary linear group over the field $\operatorname{GF}(q)$. If $G$ is primitive on $V$, then it follows from the main result of [Ph2] (or see [Ph1, Theorem 10.12.2]) that $\bar{G}^{(6)}$ is infinite simple. This is of course the primitive case described in (i) above. Note that $\bar{M}=\bar{G}^{(6)}$ is infinite, since $\bar{G}$ is clearly infinite and $\bar{G} / \bar{M} \subseteq \operatorname{Out}(\bar{M})$.

Next, if $G$ is imprimitive on $V$, then we can write $V=\prod_{i \in \mathcal{I}} V_{i}$ as a nontrivial (weak) direct product of subgroups permuted transitively by $G$. Since $G=H^{G}$, $H$ must act nontrivially in its permutation action on the factors $V_{i}$ and hence it has a nontrivial orbit. In particular, if $\left|V: \mathbb{C}_{V}(H)\right|=k$, then Lemma 3.3 implies that each $V_{i}$ has order $\leq k$ and that $H$ centralizes all but finitely many of the $V_{i}$ s. Now the bound on $\left|V_{i}\right|$ implies that we can choose this situation with the $V_{i}$ s having maximal possible order. It is then clear that $G$ permutes the $V_{i}$ s in a primitive manner, since otherwise the factors could be combined into larger groups corresponding to nontrivial blocks of imprimitivity. Consequently, $G / L \subseteq \operatorname{Sym}_{\mathcal{I}}$ is a primitive permutation group. Furthermore, since $H$ moves at most finitely many points of $\mathcal{I}$ and since $G=H^{G}$, it follows that $G / L$ is finitary in its permutation action and hence $G / L \subseteq \operatorname{FSym}_{\mathcal{I}}$. Note that $V$ is infinite and that each $V_{i}$ is finite, so $\mathcal{I}$ is infinite. Thus by [W2, Satz 9.4] (or see [W3, page 228]) it follows that $G / L=\mathrm{FSym}_{\mathcal{I}}$ or $\mathrm{FAlt}_{\mathcal{I}}$.

For the structure of $L$, we first observe that $L$ stabilizes each $V_{i}$. Furthermore, since $L$ is finitary on $V$, it is clear that each element of $L$ can act nontrivially on at most finitely many of the $V_{i}$. Thus the action of $L$ on $V$ yields an embedding of $L / N$ into the (weak) direct product $\prod_{i \in \mathcal{I}} \operatorname{Aut}\left(V_{i}\right)$. In particular, since the latter direct product is an f.c. group, so is $L / N$.

Finally, if $V$ is semisimple, then $V=\prod_{i \in \mathcal{I}} V_{i}$ is a (weak) direct product of finite nonabelian simple groups. Moreover, since the $V_{i}$ s are the minimal normal subgroups of $V$, they are permuted transitively by $G$. Again, this is an imprimitive situation and the above argument yields the result.

Note that $\mathrm{FAlt}_{\mathcal{I}}$ is an infinite simple group which is a finitary linear group over any field. Furthermore, $\mathrm{FAlt}_{\mathcal{I}} \triangleleft \mathrm{FSym}_{\mathcal{I}}$ with factor group of order 2 . With this, we can now obtain

Proposition 5.5. Let $G$ act faithfully and in a strongly finitary manner on the locally finite f.c. group $V$. If $G$ is the normal closure of a finite subgroup $H$, then $G$ has a finite subnormal series

$$
1=G_{0} \triangleleft G_{1} \triangleleft \cdots \triangleleft G_{m}=G
$$

with each quotient $G_{i} / G_{i-1}$ either infinite simple or an f.c. group. Furthermore, each such infinite simple group is a finitary linear group over a finite field $\mathrm{GF}(q)$ for some prime $q$ involved in $V$.

Proof. By Lemma 5.1, $V$ has a finite chain

$$
1=V_{0} \subseteq V_{1} \subseteq \cdots \subseteq V_{k}=V
$$

of $G$-stable subgroups such that, for each $i$, either $G$ acts irreducibly on $\bar{V}_{i}=V_{i} / V_{i-1}$ or it acts trivially on this quotient. We prove the proposition by induction on $k$, the case $k=0$ being obvious.

Suppose now that $k \geq 1$ and let $N \triangleleft G$ be the kernel of the action of $G$ on $V / V_{1}$. Then, by induction, $G / N$ has a finite subnormal series with factors as described above. Next, let ${ }^{\sim}: G \rightarrow G / \mathbb{C}_{G}\left(V_{1}\right)$ be the natural epimorphism. Then 
$\tilde{G}$ acts faithfully on $V_{1}$ and certainly $\tilde{G}=\tilde{H}^{\tilde{G}}$. If $\tilde{G}$ acts irreducibly on $V_{1}$ and if $V_{1}$ is infinite, then the preceding proposition implies that $\tilde{G}$ has an appropriate subnormal series. On the other hand, if $G$ acts trivially on $V_{1}$ or if $V_{1}$ is finite, then $\tilde{G}$ is finite and again this group has a suitable series. Futhermore, since $\tilde{N} \triangleleft \tilde{G}$, it follows that $\tilde{N}$ also has a finite subnormal series with factors which are either infinite simple or f.c. groups. Finally, observe that $\mathbb{C}_{N}\left(V_{1}\right)$ stabilizes the chain $1 \subseteq V_{1} \subseteq V$ and acts trivially on each factor. Thus, since $\mathbb{C}_{N}\left(V_{1}\right)$ acts faithfully on $V$, it must be abelian and, by combining this fact with the subnormal series for $G / N$ and for $\tilde{N}=N / \mathbb{C}_{N}\left(V_{1}\right)$, the result follows.

We remark that the locally finite, simple finitary linear groups are completely described in [H1] and [H2], and that the full force of this characterization was crucial to the proof of the main result of [P10]. Here, we just use a minor consequence of this characterization to show

Lemma 5.6. If $G$ is a locally finite, simple finitary linear group and if $H$ is a nonidentity finite subgroup of $G$, then $G=H^{[G]}$.

Proof. It follows from [KWe, Proposition 4.6] and the above mentioned results of [H1, Theorem 1.3] and [H2] that $G$ has a local system $\mathcal{L}$ consisting of finite quasisimple groups. Indeed, $\mathcal{L}$ is a Kegel system (see [KWe, Section $4 \mathrm{~A}]$ ) which, in this context, implies that if $L_{1} \in \mathcal{L}$, then there exists $L_{2} \in \mathcal{L}$ with $L_{2} \supseteq L_{1}$ and $L_{1} \cap \mathbb{Z}\left(L_{2}\right)=1$.

Now let $L_{1} \in \mathcal{L}$ with $L_{1} \supseteq H$ and let $L_{2} \in \mathcal{L}$ be as above. Then $H$ is not contained in $\mathbb{Z}\left(L_{2}\right)$ so, since $L_{2}$ is quasi-simple, it follows from Lemma 2.7(i) that $H^{\left[L_{2}\right]}=L_{2} \supseteq L_{1}$. Thus $H^{[G]} \supseteq L_{1}$ and, since $L_{1} \in \mathcal{L}$ is arbitrary, we conclude that $H^{[G]}=G$.

It is certainly reasonable to suspect that the preceding lemma should hold for all locally finite simple groups. However, the following clever example of Ulrich Meierfrankenfeld shows that this is not the case.

Lemma 5.7. Let $G$ be a locally finite simple group which is not absolutely simple. If $H$ is any finite subgroup of $G$, then $H^{[G]} \neq G$.

Proof. By definition, $G$ has an ascending subnormal series $G_{0} \triangleleft G_{1} \triangleleft G_{2} \triangleleft \cdots$ of proper subgroups with $G=\bigcup_{i} G_{i}$. Now let $H$ be any finite subgroup of $G$, so that $H \subseteq G_{n}$ for some $n$. If $L$ is a finite subgroup of $G$ containing $H$, then $L \subseteq G_{m}$ for some $m \geq n$ and we have

$$
H \subseteq L \cap G_{n} \triangleleft L \cap G_{n+1} \triangleleft \cdots \triangleleft L \cap G_{m}=L .
$$

Thus $H^{[L]} \subseteq L \cap G_{n} \subseteq G_{n}$ and, since $L$ is arbitrary, we conclude that $H^{[G]} \subseteq G_{n}$ and therefore that $H^{[\bar{G}]} \neq G$.

Note that [M, Section 6] proves the existence of locally finite simple groups which are not absolutely simple.

\section{A Critical Situation}

As usual, all groups considered here are assumed to be locally finite. For a fixed prime $p$, we say that the triple $(G, C, H)$ is critical if

1. $C \triangleleft G$ and $G / C$ is an infinite simple group.

2. $H=H^{p}$ is a finite subgroup of $G$ with $G=H^{G}$. 
3. $H \subseteq \mathbb{S}(C H), \mathbb{O}_{p}(G)=1$ and $G=\mathbb{D}_{G}(\mathbb{F}(G))$.

Our goal in this section is to obtain a fairly tight structure theorem for such critical triples. As will be apparent, this is fairly easy if $G / C$ is not a finitary alternating group. On the other hand, the latter group seems to require a good deal of additional work, both here and in our later ring theoretic considerations.

For any integer $n$, we define

$$
\left.\mathbb{S}_{n}(G)=\langle A| A \operatorname{lsn} G \text { and } \operatorname{len}(A) \leq n\right\rangle,
$$

where $\operatorname{len}(A)$ denotes the composition length of $A$. Furthermore, we let

$$
\left.\mathbb{S}_{n}^{p}(G)=\langle A| A \operatorname{lsn} G, \operatorname{len}(A) \leq n \text { and } A=A^{p}\right\rangle .
$$

As in Lemma 4.1 (ii), it is clear that $\mathbb{S}_{n}^{p}(G)$ is the subgroup of $\mathbb{S}_{n}(G)$ generated by its $p$-elements, and certainly these are all characteristic subgroups of $G$. The following is a slight extension of the main result of [P3].

Proposition 6.1. Let $G$ be a locally finite group with $\mathbb{O}_{p}(G)$ finite. Then $\mathbb{S}^{p}(G)$ is the ascending union of the f.c. groups $\mathbb{S}_{n}^{p}(G)$.

Proof. We must show that each $\mathbb{S}_{n}^{p}(G)$ is an f.c. group. To this end, let ${ }^{-}: G \rightarrow$ $G / \mathbb{O}_{p}(G)=\bar{G}$ denote the natural epimorphism. If $X=\mathbb{S}_{n}^{p}(G)$, then it is clear that $\bar{X} \subseteq \mathbb{S}_{n}^{p}(\bar{G})$, and note that $\mathbb{S}_{n}^{p}(\bar{G})$ is an f.c. group by [P3, Corollary 2] since $\mathbb{O}_{p}(\bar{G})=1$. Thus $\bar{X}$ is an f.c. group and hence it is generated by finite normal subgroups. Since $\mathbb{O}_{p}(G)$ is finite and $\bar{X} \cong X /\left(X \cap \mathbb{O}_{p}(G)\right)$, it follows that $X$ is also generated by finite normal subgroups, and consequently $X$ is an f.c. group.

We now begin our study of critical triples.

Lemma 6.2. If $(G, C, H)$ is a critical triple, then $G$ has a finite normal series

$$
1=C_{0} \subseteq C_{1} \subseteq \cdots \subseteq C_{s}=C \subseteq G
$$

such that, for each $i, G$ acts either irreducibly or trivially on the quotient group $\bar{C}_{i}=C_{i} / C_{i-1}$. Furthermore, each such action is finitary, so each $\bar{C}_{i}$ is an f.c. group and no infinite simple group can be involved in $C$.

Proof. Since $C \triangleleft G$, we have $\mathbb{O}_{p}(C)=1$ and hence, since $|C H: C|<\infty$, it follows that $\mathbb{O}_{p}(C H)$ is finite. Now $H=H^{p} \subseteq \mathbb{S}(C H)$, so there exists an integer $n$ with $H \subseteq \mathbb{S}_{n}^{p}(C H)=X$. Note that $X$ is an f.c. group by Proposition 6.1 , since $\mathbb{O}_{p}(C H)$ is finite. Thus there exists a finite normal subgroup $N$ of $X$ with $H \subseteq N$. Furthermore, since $C N=C H \supseteq X \supseteq N$, it follows that $X=(C \cap X) N$ and, of course, $C \cap X \triangleleft C H$. We study $C \cap X$ via the isomorphism

$$
\bar{X}=\frac{X}{N}=\frac{(C \cap X) N}{N} \cong \frac{C \cap X}{C \cap N} .
$$

To this end, let $A$ be a generator of $X=\mathbb{S}_{n}^{p}(C H)$ so that $A \operatorname{lsn} C H, A=A^{p}$ and $\operatorname{len}(A) \leq n$. Then $\bar{A} \operatorname{lsn} \bar{X}, \bar{A}=\bar{A}^{p}$ and $\operatorname{len}(\bar{A}) \leq n$. In particular, if $B$ is the complete inverse image of $\bar{A}$ in $C \cap X$, with respect to the preceding displayed isomorphism, then $B \operatorname{lsn}(C \cap X)$ and $\operatorname{len}(B) \leq n+k$ where $k=\operatorname{len}(C \cap N)$. Furthermore, note that $B^{p}$ maps onto $\bar{A}^{p}=\bar{A}$ and that $B^{p} \operatorname{lsn}(C \cap X)$ with $\operatorname{len}\left(B^{p}\right) \leq \operatorname{len}(B) \leq n+k$. Consequently, since $C \cap X \triangleleft C$, we have $B^{p} \subseteq \mathbb{S}_{n+k}^{p}(C)=$ $D$. Indeed, since the $\bar{A}$ s generate $\bar{X}$, it follows that $C \cap X \subseteq D(C \cap N)$. Certainly $D$, as defined here, is a characteristic subgroup of $C$, and hence $D \triangleleft G$. Finally, $H \subseteq \mathbb{S}_{n}^{p}(C H) \subseteq \mathbb{S}_{n+k}^{p}(C H)$ and clearly $D=\mathbb{S}_{n+k}^{p}(C) \subseteq \mathbb{S}_{n+k}^{p}(C H)$. Thus, since 
$\mathbb{S}_{n+k}^{p}(C H)$ is an f.c. group, by the preceding theorem, it follows that $H \subseteq \mathbb{D}_{G}(D)$. But $\mathbb{D}_{G}(D) \triangleleft G$, so this group must also contain $H^{G}=G$, and hence $G=\mathbb{D}_{G}(D)$.

Next, since $H \subseteq X \triangleleft C H$, we have $[C, H] \subseteq C \cap X \subseteq D(C \cap N)$. Thus, $[C, H]$ is finite modulo $D$, so $H D / D \subseteq \mathbb{D}_{G / D}(C / D) \triangleleft G / D$. Again, using $H^{G}=G$, we conclude that $G / D=\mathbb{D}_{G / D}(C / D)$. In particular, both $D$ and $C / D$ are f.c. groups, and therefore no infinite simple group can be involved in $C$.

For the remaining part, note that $D$ is an f.c. group and that $G=\mathbb{D}_{G}(D)$, so $G$ acts on $D$ in a strongly finitary manner. Thus, Lemma 5.1 implies that $D$ has a finite normal series of $G$-stable subgroups such that $G$ acts irreducibly or trivially on each factor group. Furthermore, each such factor is an f.c. group since $D$ is an f.c. group. Similarly, using $G / D=\mathbb{D}_{G / D}(C / D)$, we see that there is a finite normal series from $D$ to $C$ of $G$-stable subgroups such that $G$ acts trivially or irreducibly on each quotient. By combining these two series, the result follows.

If $\bar{C}_{i}=C_{i} / C_{i-1}$ is an infinite irreducible $G$-module, then it is either primitive or imprimitive, as described in Proposition 5.4. Furthermore, if some $\bar{C}_{i}$ is imprimitive, then $G$ must involve the finitary alternating group $\mathrm{FAlt}_{\mathcal{I}}$ for some infinite set $\mathcal{I}$ and, in view of the preceding result, we must have $G / C \cong \mathrm{FAlt}_{\mathcal{I}}$. As we mentioned earlier, these are the only infinite simple groups which require additional work. Indeed, the following lemma is sufficient to handle the primitive case.

Lemma 6.3. Let $(G, C, H)$ be a critical triple and use the notation of the preceding lemma. Then there exists a subgroup $\tilde{G}$ of $G$ with the following properties.

i. $\tilde{G} \triangleleft G$ and $\left|G^{(6)}: \tilde{G}\right|<\infty$.

ii. $\tilde{G}$ acts trivially on all those $\bar{C}_{i}$ which are either finite or trivial $G$-modules.

iii. $\tilde{C}=C \cap \tilde{G}$ acts trivially on all those $\bar{C}_{i}$ which are not infinite, irreducible and imprimitive $G$-modules.

iv. $\tilde{G} C=G$ and therefore $\tilde{G} / \tilde{C} \cong G / C$ is infinite simple.

In particular, if all infinite irreducible $\bar{C}_{i}$ are primitive $G$-modules, then $\tilde{C}$ is a nilpotent $p^{\prime}$-group.

Proof. Let $\bar{C}_{i}$ be an infinite, irreducible and primitive $G$-module. If $\theta_{i}: G \rightarrow$ Aut $\left(\bar{C}_{i}\right)$ denotes the corresponding representation, then Proposition 5.4(i) implies that $\theta_{i}\left(G^{(6)}\right)$ is infinite simple. Furthermore, since $C \cap G^{(6)} \triangleleft G^{(6)}$ and since this group cannot involve an infinite simple group by the preceding lemma, it follows that $\theta_{i}\left(C \cap G^{(6)}\right)=1$. In other words, $C \cap G^{(6)}$ acts trivially on all $\bar{C}_{i}$ which are infinite irreducible and primitive, and of course it acts trivially on all $\bar{C}_{i}$ which are trivial $G$-modules.

Now let $J \triangleleft G$ be the kernel of the action of $G$ on all finite $\bar{C}_{i}$. Then $G / J$ is finite and hence $\tilde{G}=G^{(6)} \cap J$ is a normal subgroup of $G$ having finite index in $G^{(6)}$. Furthermore, by the above, $\tilde{C}=C \cap \tilde{G}=\left(C \cap G^{(6)}\right) \cap J$ is a subgroup of $C$ which acts trivially on all those $\bar{C}_{i}$ which are not infinite, irreducible and imprimitive $G$-modules. This proves the first three parts, and the fourth is immediate. Indeed, since $G / C$ is infinite simple, it follows first that $G^{(6)} C=G$ and then that $\tilde{G} C=G$. Thus $G / C=\tilde{G} C / C \cong \tilde{G} / \tilde{C}$.

Finally, if no imprimitive modules occur, then $\tilde{C} \subseteq C$ acts trivially on all $\bar{C}_{i}$ and this clearly implies that $\tilde{C}$ is nilpotent. But $\mathbb{O}_{p}(G)=1$, so $\mathbb{O}_{p}(\tilde{C})=1$ and hence $\tilde{C}$ is a $p^{\prime}$-group. 
The final observation above is sufficient to handle the Jacobson radical problem in the primitive case, since the main result of [P10] guarantees that any characteristic $p$ twisted group algebra of such a group $\tilde{G}$ is necessarily semiprimitive.

The goal now is to obtain a better understanding of the subgroup $\tilde{C}$ when imprimitive $G$-modules occur. To this end, let $\mathcal{I}$ be an infinite set of cardinality equal to that of $G / C$ and let $\mathfrak{A}=$ FAlt $_{\mathcal{I}}$ denote the finitary alternating group on $\mathcal{I}$. Furthermore, if $i \in \mathcal{I}$, let $\mathfrak{A}_{i}$ denote the stabilizer of $i$ in $\mathfrak{A}$, so that $\mathfrak{A}_{i} \cong \mathfrak{A}$. The following two results isolate the basic properties of $\mathfrak{A}$-modules which we require.

Lemma 6.4. Let $C \triangleleft G$ with $G / C \cong \mathfrak{A}=\mathrm{FAlt}_{\mathcal{I}}$ and let $V=\prod_{i \in \mathcal{I}} V_{i}$ be the (weak) direct product of the finite groups $V_{i}$. Suppose that $G$ acts on $V$ in such a way that $\left(V_{i}\right)^{g}=V_{i g}$ where $i g$ is the image of $i \in \mathcal{I}$ under the permutation $C g \in \mathfrak{A}$. Now let $L$ be a $G$-stable subgroup of $V$ and define $L_{i, j}=L \cap V_{i} V_{j}$ for all distinct $i, j \in \mathcal{I}$. Then we have

i. $L_{i, j}^{g}=L_{i g, j g}$ and $L_{j, i}=L_{i, j} \triangleleft L$.

ii. $L_{i, j} \subseteq L_{i, k} L_{k, j}$ for all distinct $i, j, k \in \mathcal{I}$.

iii. $L=\left\langle L_{i, j} \mid i, j \in \mathcal{I}\right\rangle$.

Proof. Part (i) is obvious. For the remainder, let $v=v_{1} v_{2} v_{3} \cdots v_{n} \in L$ with $v_{i} \in V_{i}$, and let $k$ and $k^{\prime}$ be distinct elements of $\mathcal{I}$ different from $1,2, \ldots, n$. If $S=L_{1, k} L_{2, k} \cdots L_{n, k}$, then we claim that $v \in S$. To this end, let $B / C=\mathfrak{B}=$ $\mathfrak{A}_{2} \cap \mathfrak{A}_{3} \cap \cdots \cap \mathfrak{A}_{n}$ and observe that $B$ stabilizes the finite group $W=V_{2} V_{3} \cdots V_{n}$. Thus, $B$ has a subgroup $B_{0}$ of finite index which centralizes $W$. But $\mathfrak{B} \cong \mathfrak{A}$ has no proper subgroup of finite index, so $B_{0}$ maps onto $\mathfrak{B}$ and, in particular, there exists $g \in B_{0}$ which corresponds to the 3 -cycle $\left(1 k k^{\prime}\right)$. Since $g \in B_{0}$ centralizes $v_{2}, v_{3}, \ldots, v_{n}$, we have $v^{g}=u_{1} v_{2} v_{3} \cdots v_{n} \in L$ where $u_{1}=v_{1}^{g} \in V_{k}$, and therefore

$$
v_{1} u_{1}^{-1}=v v^{-g} \in L \cap V_{1} V_{k}=L_{1, k} .
$$

Similarly, there exist elements $u_{2}, u_{3}, \ldots, u_{n} \in V_{k}$ with $v_{i} u_{i}^{-1} \in L_{i, k}$ and, by multiplying these in order, we obtain

$$
v u=v_{1} v_{2} \cdots v_{n} u \in S \subseteq L
$$

where $u=u_{1}^{-1} u_{2}^{-1} \cdots u_{n}^{-1} \in V_{k}$. But $v \in L$, so $u \in L \cap V_{k} \subseteq L \cap V_{n} V_{k}=L_{n, k}$, and therefore $v \in S$, as required.

This, of course, proves part (iii) since every element of $L$ is of the given form. Furthermore, with $n=2$, the above yields $L_{1,2}=L \cap V_{1} V_{2} \subseteq L_{1, k} L_{2, k}$, and the result follows.

As is well known, $L$ need not be generated by its subgroups $L \cap V_{i}$. Indeed, let $G=\mathfrak{A}$ and let $V$ be the natural permutation module for $G$ over any finite field $F$ (in additive notation). If $L$ denotes the set of all elements of $V$ with coefficient sum 0 , then $L$ is a $G$-submodule of $V$ with $L \cap V_{i}=0$ for all $i \in \mathcal{I}$. Thus $L$ is certainly not generated by these intersections. Now we need a converse of sorts.

Lemma 6.5. Let $C \triangleleft G$ with $G / C \cong \mathfrak{A}=$ FAlt $_{\mathcal{I}}$ and let $G$ act on the group $V$. Suppose that $V$ contains a family $\left\{L_{i, j} \mid i, j \in \mathcal{I}, i \neq j\right\}$ of finite normal subgroups such that

1. $\left(L_{i, j}\right)^{g}=L_{i g, j g}$ where $i g$ is the image of $i \in \mathcal{I}$ under the permutation $C g \in \mathfrak{A}$, and

2. $L_{j, i}=L_{i, j} \subseteq L_{i, k} L_{k, j}$ for all distinct $i, j, k \in \mathcal{I}$. 
If $L=\left\langle L_{i, j} \mid i, j \in \mathcal{I}\right\rangle$, then $V$ has $G$-stable normal subgroups $D \subseteq X \subseteq L$ and finite normal subgroups $X_{i}$ for $i \in \mathcal{I}$ with the following properties.

i. $D$ is finite and $L / X$ is abelian.

ii. $D \subseteq X_{i} \subseteq X$ and $X / D$ is the (weak) direct product $\prod_{i \in \mathcal{I}}\left(X_{i} / D\right)$.

iii. $\left(X_{i}\right)^{g}=X_{\text {ig }}$ for all $i \in \mathcal{I}$ and $g \in G$.

Furthermore, we have

iv. $\left[X_{i}, L_{j, k}\right] \subseteq D$ and $X_{j} \subseteq L_{j, k}$ for all distinct $i, j, k \in \mathcal{I}$.

Proof. First let $i, j, a, b, a^{\prime}, b^{\prime}$ be six distinct elements of $\mathcal{I}$ and let $B / C=\mathfrak{B}=$ $\mathfrak{A}_{i} \cap \mathfrak{A}_{j}$. Then $B$ stabilizes the finite group $L_{i, j}$, and therefore some subgroup $B_{0}$ of finite index in $B$ centralizes $L_{i, j}$. Moreover, since $\mathfrak{B} \cong \mathfrak{A}$ has no proper subgroup of finite index, it follows that $B_{0}$ maps onto $\mathfrak{B}$. In particular, there exists an element $g \in B_{0}$ which corresponds to the permutation $\left(a a^{\prime}\right)\left(b b^{\prime}\right)$. Since $g \in B_{0}$ centralizes $L_{i, j}$, we conclude that

$$
L_{i, j} \cap L_{a, b}=\left(L_{i, j} \cap L_{a, b}\right)^{g}=L_{i g, j g} \cap L_{a g, b g}=L_{i, j} \cap L_{a^{\prime}, b^{\prime}} .
$$

It is now easy to see that all such intersections are equal.

To start with, let $\{1,2,3,4\}$ and $\{a, b, c, d\}$ be disjoint subsets of $\mathcal{I}$, each of size 4. Then, by the above, we have

$$
L_{a, b} \cap L_{c, d}=L_{a, b} \cap L_{3,4}=L_{1,2} \cap L_{3,4} .
$$

Finally, let $\{a, b, c, d\}$ be an arbitrary set of size 4 and let $\left\{a^{\prime}, b^{\prime}, c^{\prime}, d^{\prime}\right\}$ be disjoint from this set and from $\{1,2,3,4\}$. Then

$$
L_{a, b} \cap L_{c, d}=L_{a^{\prime}, b^{\prime}} \cap L_{c^{\prime}, d^{\prime}}=L_{1,2} \cap L_{3,4} .
$$

In particular, if we set $D=L_{1,2} \cap L_{3,4}$, then $D$ is a finite normal subgroup of $V$ and $L_{a, b} \cap L_{c, d}=D$ for all subsets $\{a, b, c, d\}$ of $\mathcal{I}$ of size 4 . By (1), it is clear that $D$ is $G$-stable.

Now we repeat this argument, but this time with sets of size 2 . To start with, let $i, a, b, a^{\prime}, b^{\prime}, c$ be distinct members of $\mathcal{I}$ and let $B / C=\mathfrak{B}=\mathfrak{A}_{i} \cap \mathfrak{A}_{a}$. Then $B$ has a subgroup $B_{0}$ of finite index which centralizes $L_{i, a}$ and therefore there exists $g \in B_{0}$ which corresponds to the 3 -cycle $\left(\begin{array}{lll}b & b^{\prime} & c\end{array}\right)$. Since $g$ centralizes $L_{i, a}$, we have

$$
L_{i, a} \cap L_{i, b}=\left(L_{i, a} \cap L_{i, b}\right)^{g}=L_{i g, a g} \cap L_{i g, b g}=L_{i, a} \cap L_{i, b^{\prime}},
$$

and it is now easy to see that all such intersections, for a fixed $i$, are equal.

Indeed, let $\{1,2\}$ and $\{a, b\}$ be disjoint subsets of $\mathcal{I} \backslash\{i\}$ of size 2 . Then, by the above, we have

$$
L_{i, a} \cap L_{i, b}=L_{i, a} \cap L_{i, 2}=L_{i, 1} \cap L_{i, 2} .
$$

Furthermore, if $\{a, b\}$ is any subset of size 2 not containing $i$, choose another such set $\left\{a^{\prime}, b^{\prime}\right\}$ which is disjoint from it and from $\{1,2\}$. Then

$$
L_{i, a} \cap L_{i, b}=L_{i, a^{\prime}} \cap L_{i, b^{\prime}}=L_{i, 1} \cap L_{i, 2} .
$$

In particular, if we let $X_{i}=L_{i, 1} \cap L_{i, 2}$, then $X_{i}=L_{i, a} \cap L_{i, b}$ for all sets $\{i, a, b\}$ of size 3. It follows that $X_{i}$ is a finite normal subgroup of $V$, and assumption (1) yields $\left(X_{i}\right)^{g}=X_{i g}$.

Furthermore, if $i, j, k$ are distinct, then

$$
\begin{aligned}
{\left[X_{i}, L_{j, k}\right] \subseteq X_{i} \cap L_{j, k} } & =L_{i, 1} \cap L_{i, 2} \cap L_{j, k} \\
& =\left(L_{i, 1} \cap L_{j, k}\right) \cap\left(L_{i, 2} \cap L_{j, k}\right)=D \cap D=D,
\end{aligned}
$$


where we chose 1,2 to be distinct elements of $\mathcal{I} \backslash\{i, j, k\}$. In particular, we now know that $X_{i} \supseteq D$ and, since $X_{j}=L_{j, k} \cap L_{j, k^{\prime}}$, it follows that

$$
X_{i} \cap X_{j}=\left(X_{i} \cap L_{j, k}\right) \cap\left(X_{i} \cap L_{j, k^{\prime}}\right)=D \cap D=D .
$$

For the remainder of the proof, let $X=\left\langle X_{i} \mid i \in \mathcal{I}\right\rangle$, so that $X$ is a $G$-stable normal subgroup of $V$ with $D \subseteq X \subseteq L$.

To show that $L / X$ is abelian, consider $L_{i, j}$. By assumption (2), we see that $L$ is generated by all $L_{a, b}$ with $\{a, b\} \neq\{i, j\}$. Since $\left[L_{i, j}, L_{a, b}\right] \subseteq L_{i, j} \cap L_{a, b} \subseteq X$ by the preceding considerations, it follows that $\left[L_{i, j}, L\right] \subseteq X$ and thus $[L, L] \subseteq X$.

Finally, set $X / D=\bar{X}$ and $X_{i} / D=\bar{X}_{i}$. Furthermore, let $V_{i}$ be another copy of $\bar{X}_{i}$ and let $V=\prod_{i \in \mathcal{I}} V_{i}$ be the external (weak) direct product of the $V_{i}$ s. Since $\bar{X}_{i} \cap \bar{X}_{j}=1$ for $i \neq j$, these generating subgroups of $\bar{X}$ centralize each other, and thus there exists a natural epimorphism $\theta: V \rightarrow \bar{X}$ with $\theta\left(V_{i}\right)=\bar{X}_{i}$. Moreover, since $G$ acts on $\bar{X}$ by permuting the $\bar{X}_{i}$ s, there is an obvious action of $G$ on $V$ which makes $\theta$ a $G$-homomorphism. In particular, $\operatorname{ker} \theta$ is a $G$-stable subgroup of $V$. Note that $\bar{X}_{i} \cap \bar{X}_{j}=1$ implies that $\bar{X}_{i} \bar{X}_{j}=\bar{X}_{i} \times \bar{X}_{j}$ is an internal direct product, and hence $\operatorname{ker} \theta \cap V_{i} V_{j}=1$. Lemma 6.4 (iii) now implies that $\operatorname{ker} \theta=1$, so $\theta$ is an isomorphism and $X / D$ is the (weak) direct product $\prod_{i \in \mathcal{I}}\left(X_{i} / D\right)$.

Obviously, more can be said about the abelian quotient $L / X$, but that additional information will not be needed here. We can now obtain our main result on critical triples. For this, it is convenient to begin with a brief discussion of two facts which are required for its proof.

First, if a group $G$ is generated by the family of normal subgroups $\left\{N_{i} \mid i \in \mathcal{I}\right\}$, then $G^{p}$ is generated by the normal subgroups $N_{i}^{p}$. Indeed, if $M=\left\langle N_{i}^{p} \mid i \in \mathcal{I}\right\rangle$, then $M \triangleleft G$ and $M \subseteq G^{p}$. Furthermore, since $N_{i} \cap M \supseteq N_{i}^{p}$, it follows that $G / M$ is generated by the normal $p^{\prime}$-subgroups $N_{i} /\left(N_{i} \cap M\right)$. Thus $G / M$ is a $p^{\prime}$-group and $G^{p}=M$, as required.

Second, we note that any isomorphism $\sigma:$ FAlt $_{\mathcal{I}} \rightarrow$ FAlt $_{\mathcal{K}}$ with $|\mathcal{I}|>6$ must arise from a one-to-one correspondence $\sigma^{*}$ between the elements of $\mathcal{I}$ and those of $\mathcal{K}$. Indeed, the existence of $\sigma$ implies that $\mathcal{I}$ and $\mathcal{K}$ necessarily have the same cardinality. Thus there exists a one-to-one correspondence $\tau^{*}: \mathcal{I} \rightarrow \mathcal{K}$ and this gives

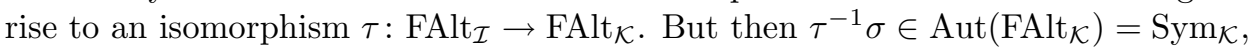
by [Sc, Theorem 11.4.8(4)], so $\tau^{-1} \sigma$ arises from a permutation $\eta^{*} \in \mathrm{Sym}_{\mathcal{K}}$, and consequently $\sigma=\tau\left(\tau^{-1} \sigma\right)$ arises from the one-to-one correspondence $\tau^{*} \eta^{*}: \mathcal{I} \rightarrow \mathcal{K}$.

Proposition 6.6. For a fixed prime p, let $(G, C, H)$ be a critical triple and, in the notation of Lemma 6.2, assume that some $\bar{C}_{r}$ is an infinite irreducible and imprimitive $G$-module. Then $G / C \cong \mathfrak{A}=\mathrm{FAlt}_{\mathcal{I}}$ for some infinite set $\mathcal{I}$, and $G$ has normal subgroups $\tilde{D} \subseteq \tilde{X} \subseteq \tilde{L} \subseteq \tilde{C} \subseteq \tilde{G}$ with the following properties.

i. $\left|G^{(6)}: \tilde{G}\right|<\infty, \tilde{C}=C \cap \tilde{G}, \tilde{G} C=G$, and $\tilde{G} / \tilde{C} \cong G / C \cong \mathfrak{A}$.

ii. $\tilde{L}=\tilde{C}^{p}$, so that $\tilde{C} / \tilde{L}$ is a $p^{\prime}$-group.

iii. $\tilde{D}$ is a finite abelian $p^{\prime}$-group which is central in $\tilde{G}^{p}$.

iv. $\tilde{L}$ is an f.c. group, and $\tilde{L} / \tilde{X}$ is an abelian p-group.

v. There exist finite normal subgroups $\tilde{X}_{i}$ of $\tilde{C}$, for all $i \in \mathcal{I}$, with $\left(\tilde{X}_{i}\right)^{g}=\tilde{X}_{i g}$ where $i g$ is the image of $i \in \mathcal{I}$ under the permutation $C g \in \mathfrak{A}$.

vi. $\tilde{D} \subseteq \tilde{X}_{i} \subseteq \tilde{X}$ and $\tilde{X} / \tilde{D}$ is the (weak) direct product $\prod_{i \in \mathcal{I}}\left(\tilde{X}_{i} / \tilde{D}\right)$.

Furthermore, we have

vii. $\tilde{X}_{j} / \tilde{D} \subseteq\left(\mathbb{C}_{\tilde{L} / \tilde{D}}\left(\tilde{X}_{i} / \tilde{D}\right)\right)^{p}$ for all distinct $i, j \in \mathcal{I}$. 
Proof. Since some $\bar{C}_{r}$ is an infinite irreducible and imprimitive $G$-module, we know that $G / C \cong \mathfrak{A}=\mathrm{FAlt}_{\mathcal{I}}$, where we take $\mathcal{I}$ to be a fixed infinite set of the same cardinality as that of $G / C$. Furthermore, we fix the preceding isomorphism, thereby identifying $G / C$ with $\mathfrak{A}$. Let $\tilde{G}$ be the normal subgroup of $G$ given by Lemma 6.3 and observe that (i) is now satisfied.

Suppose $\bar{C}_{r}$ is any infinite irreducible and imprimitive $G$-module which occurs in the series given by Lemma 6.2. Then by Proposition 5.4(ii), $\bar{C}_{r}$ is the (weak) direct product $\prod_{k \in \mathcal{K}} W_{k}$ of the finite subgroups $W_{k}$ and $G$ permutes these factors as the finitary alternating or symmetric group on $\mathcal{K}$. In particular, since $C \triangleleft G$ cannot involve an infinite simple group, $C$ must be in the kernel of this permutation action, and consequently we obtain an isomorphism $G / C=\mathrm{FAlt}_{\mathcal{I}} \cong \mathrm{FAlt}_{\mathcal{K}}$. But, as we observed above, any such isomorphism $\mathrm{FAlt}_{\mathcal{I}} \cong \mathrm{FAlt}_{\mathcal{K}}$ must arise from a one-toone correspondence between the elements of $\mathcal{I}$ and those of $\mathcal{K}$. In other words, we can reindex the factors $W_{k}$ of $\bar{C}_{r}$ with the elements of the set $\mathcal{I}$ in such a way that $G / C=$ FAlt $_{\mathcal{I}}$ permutes these factors in the natural manner. We now suitably reindex each such $\bar{C}_{r}$ which occurs.

Let $V$ be the direct sum of the finitely many $\bar{C}_{r}$ s which are infinite irreducible and imprimitive $G$-modules. By the above observation, we can combine the factors of the various $\bar{C}_{r}$ s corresponding to the same index $i \in \mathcal{I}$ and write $V=\prod_{i \in \mathcal{I}} V_{i}$ as a (weak) direct product of finite normal subgroups which are permuted by $G$ via its homomorphism to the finitary alternating group $G / C=\mathfrak{A}=\mathrm{FAlt}_{\mathcal{I}}$. Furthermore, since $C$ is the kernel of this homomorphism, $C$ stabilizes each $V_{i}$ and, by Lemma 6.2, $C$ is finitary in its action on $V$. Let $\tilde{T}$ denote the kernel of the action of $\tilde{C}$ on $V$, so that $\tilde{T}$ is certainly $G$-stable.

Now, by using the homomorphism $G \rightarrow \operatorname{Aut}(V)$, we see that $G$ acts on $\operatorname{Aut}(V)$ in a natural manner and hence it acts on the (weak) direct product $\prod_{i \in \mathcal{I}} \operatorname{Aut}\left(V_{i}\right)$. Furthermore, $\left(\operatorname{Aut}\left(V_{i}\right)\right)^{g}=\operatorname{Aut}\left(V_{i g}\right)$, each $\operatorname{Aut}\left(V_{i}\right)$ is finite, and $\tilde{C} / \tilde{T}$ is a $G$-stable subgroup of $\prod_{i \in \mathcal{I}} \operatorname{Aut}\left(V_{i}\right)$ since $\tilde{C}$ acts in a finitary manner on $V$. Thus Lemma 6.4 applies to this situation, and it follows that there are finite normal subgroups $\tilde{T}_{i, j} / \tilde{T}$ of $\tilde{C} / \tilde{T}$, for all distinct $i, j \in \mathcal{I}$, which satisfy the conclusion of that result.

By definition and Lemma 6.3(iii), $\tilde{T}$ acts trivially on all $\bar{C}_{r}$ and, since $\tilde{T} \subseteq C$, it follows that $\tilde{T}$ is nilpotent. Hence, since $\tilde{T} \triangleleft G$, we have $\mathbb{O}_{p}(\tilde{T})=1$ and thus $\tilde{T}$ is a $p^{\prime}$-group. Furthermore, $\tilde{T} \subseteq \mathbb{F}(G)$ and note that $G=\mathbb{D}_{G}(\mathbb{F}(G))$ by assumption (3) in the definition of a critical triple. Thus $\tilde{T}_{i, j} \subseteq \mathbb{D}_{G}(\tilde{T})$ and, since $\left|\tilde{T}_{i, j}: \tilde{T}\right|<\infty$, this implies that $\tilde{T}_{i, j}$ is an f.c. group. Consequently, $\tilde{T}_{i, j}=\tilde{N}_{i, j} \tilde{T}$ for some finite normal subgroup $\tilde{N}_{i, j}$ of $\tilde{T}_{i, j}$ and then $\tilde{T}_{i, j}^{p}=\tilde{N}_{i, j}^{p} \tilde{T}^{p}=\tilde{N}_{i, j}^{p}$ is finite.

Let $\tilde{L}=\tilde{C}^{p}$ so that $\tilde{L} \triangleleft G$ and $\tilde{C} / \tilde{L}$ is a $p^{\prime}$-group. Since $\tilde{C}$ is generated by its normal subgroups $\tilde{T}_{i, j}$, it follows that $\tilde{L}$ is generated by its finite normal subgroups $\tilde{L}_{i, j}=\tilde{T}_{i, j}^{p}$ and hence $\tilde{L}$ is an f.c. group. Of course, $\left(\tilde{T}_{i, j}\right)^{g}=\tilde{T}_{i g, j g}$ implies that $\left(\tilde{L}_{i, j}\right)^{g}=\tilde{L}_{i g, j g}$. Furthermore, since $\tilde{T}_{i, j} \subseteq \tilde{T}_{i, k} \tilde{T}_{k, j}$, we have

$$
\tilde{L}_{i, j}=\tilde{T}_{i, j}^{p} \subseteq\left(\tilde{T}_{i, k} \tilde{T}_{k, j}\right)^{p}=\tilde{T}_{i, k}^{p} \tilde{T}_{k, j}^{p}=\tilde{L}_{i, k} \tilde{L}_{k, j},
$$

and we can now apply the preceding lemma to the $G$-module $\tilde{L}$. This yields the subgroups $\tilde{D}, \tilde{X}$ and $\tilde{X}_{i}$, for all $i \in \mathcal{I}$, and with this, parts (ii), (v) and (vi) hold. Next, if $i, j, k$ are distinct elements of $\mathcal{I}$ then, by Lemma 6.5 (iv), $\tilde{X}_{j} \subseteq \tilde{L}_{j, k}$ and $\tilde{L}_{j, k}$ centralizes $\tilde{X}_{i}$ modulo $\tilde{D}$. Thus, since $\tilde{L}_{j, k}=\tilde{T}_{j, k}^{p}$ is generated by $p$-elements, 
part (vii) is proved. In addition, since $\tilde{L} / \tilde{X}$ is abelian and $\tilde{L}=\tilde{C}^{p}$ is generated by $p$-elements, we conclude that $\tilde{L} / \tilde{X}$ is an abelian $p$-group, as required for part (iv).

Finally, let $\tilde{D}_{r}=\tilde{D} \cap C_{r}$, where the $C_{r}$ s are the normal subgroups of $G$ defined in Lemma 6.2. Then

$$
1=\tilde{D}_{0} \subseteq \tilde{D}_{1} \subseteq \cdots \subseteq \tilde{D}_{s}=\tilde{D}
$$

is a series of normal subgroups of $G$ and $\tilde{D}_{r} / \tilde{D}_{r-1}$ is isomorphic to a normal $G$ submodule of $\bar{C}_{r}=C_{r} / C_{r-1}$. Since $\tilde{D}$ is finite, it follows that $\tilde{D}_{r} / \tilde{D}_{r-1}=1$ if $\bar{C}_{r}$ is an infinite simple $G$-module. On the other hand, if $\bar{C}_{r}$ is a trivial $G$ module or a finite module, then Lemma 6.3(ii) implies that $\tilde{G}$ acts trivially on $\bar{C}_{r}$ and hence on $\tilde{D}_{r} / \tilde{D}_{r-1}$. In particular, $\tilde{D}$ is a finite nilpotent group and, since $\mathbb{O}_{p}(G)=1$, it follows that $\tilde{D}$ is a $p^{\prime}$-group. Furthermore, if $g$ is any element of $\tilde{G}$, then $\langle\tilde{D}, g\rangle=\tilde{D}\langle g\rangle$ is also a finite nilpotent group, and therefore if $g$ is a $p$ element, then $g$ centralizes $\tilde{D}$. In other words, $\tilde{D}$ is centralized by $\tilde{G}^{p}$ and, since $\tilde{D} \subseteq \tilde{L}=\tilde{C}^{p} \subseteq \tilde{G}^{p}$, we conclude that $\tilde{D}$ is abelian. This yields part (iii), and the proposition is proved.

In Section 10, we will compute the Jacobson radical of characteristic $p$ twisted group algebras of any group $\tilde{G}$ as given above. The argument is ad hoc and requires the following two properties of finitary permutation groups.

Lemma 6.7. Let $\Omega$ be an infinite set and let $G$ be a subgroup of the finitary symmetric group $\mathrm{FSym}_{\Omega}$. If the stabilizer $G_{\Delta}=\{g \in G \mid \Delta g=\Delta\}$ of every finite subset $\Delta \subseteq \Omega$ has only infinite orbits on the complementary set $\Omega \backslash \Delta$, then $\mathbb{S}(G)=1$. In particular, if $\Omega=\Omega_{1} \cup \Omega_{2} \cup \cdots \cup \Omega_{k}$ is a disjoint union of infinite sets and if $G \supseteq$ FAlt $_{\Omega_{1}} \times$ FAlt $_{\Omega_{2}} \times \cdots \times$ FAlt $_{\Omega_{k}}$, then $\mathbb{S}(G)=1$.

Proof. Suppose by way of contradiction that $\mathbb{S}(G) \neq 1$ and let $A \neq 1$ be a minimal locally subnormal subgroup of $G$. Then $A$ is simple, so either it is a $q$-group for some prime $q$ or it is nonabelian simple. Since $A \neq 1$, it has an orbit $\mathcal{A}$ of size $>1$ which we write as the disjoint union $\mathcal{A}=\Delta \cup\{a\}$ with $\Delta \neq \emptyset$. Let $\Gamma \supseteq \mathcal{A}$ denote the finitely many points of $\Omega$ moved by $A$ and note that

$$
U=\left\{g \in G_{\Delta} \mid a g \in \Gamma \text { or } a g^{-1} \in \Gamma\right\}
$$

is a finite union of right cosets of the stabilizers in $G_{\Delta}$ of the points in $a G_{\Delta} \cap \Gamma \subseteq$ $\Omega \backslash \Delta$. Thus, since these subgroups all have infinite index in $G_{\Delta}$ by hypothesis, it follows from [P5, Lemma 4.2.1] that $U \neq G_{\Delta}$. In particular, we can choose $g \in G_{\Delta} \backslash U$, and then $a g=b$ is fixed by $A$. Furthermore, $a g^{-1}$ is fixed by $A$ and therefore $a$ is fixed by $A^{g}$. Since $\mathcal{A} g=\Delta \cup\{b\}$ is an orbit for $A^{g}$, we conclude that $\mathcal{B}=\Delta \cup\{a, b\}$ is an orbit for $B=\left\langle A, A^{g}\right\rangle$ containing $\mathcal{A}$ and of size $|\mathcal{B}|=|\mathcal{A}|+1$.

If $A$ is a $q$-group, then $B$ is a $q$-group by the local analog of Lemma 2.2(i). Hence $\mathcal{A}$ and $\mathcal{B}$ both have sizes which are powers of $q$ and this is certainly a contradiction. On the other hand, if $A$ is nonabelian simple, then the local analog of Lemma 2.9 shows that $B$ is semisimple and that $A \triangleleft B$. Since this implies that $|\mathcal{A}|$ divides $|\mathcal{B}|$, we again have a contradiction. In other words, we must have $\mathbb{S}(G)=1$, and the remaining observation is clear.

Finally, we have

Lemma 6.8. Let $\Omega$ be an infinite set and let $G$ be a transitive subgroup of $\mathrm{FSym}_{\Omega}$. If $G$ is generated by cycles of prime length, then $G=\mathrm{FSym}_{\Omega}$ or $\mathrm{FAlt}_{\Omega}$. Furthermore, the latter occurs precisely when all the prime lengths are odd. 
Proof. It suffices to show that $G$ is a primitive permutation group. To this end, let $\Delta \neq \Omega$ be a block of imprimitivity. Since $\Delta \neq \Omega$ and $G$ is transitive, there exists a generator $g \in G$, namely a $q$-cycle for some prime $q$, with $\Delta g \neq \Delta$. Thus $\Delta g \cap \Delta=\emptyset$, so $\Delta$ contains no points fixed by $g$ and therefore $\Delta$ is contained in the set $\Lambda$ of size $q$ consisting of those elements which are moved by $g$. But then $\Delta$ is a block for the permutation action of $\langle g\rangle$ on $\Lambda$ and, since the latter action is primitive and $\Delta g \cap \Delta=\emptyset$, we conclude that $|\Delta|=1$. Thus $G$ is indeed primitive and [W2, Satz 9.4] (or see [W3, page 228]) yields the result.

The preceding lemma has obvious generalizations, but this is all we require. Of course, $G$ is transitive on $\Omega$ if and only if its generating cycles are "connected" and "cover" the set $\Omega$.

\section{Twisted Group Rings}

We now move on to the ring theoretic aspects of the proof, and we start by recording several basic results on the Jacobson radical of twisted group algebras. The first, from reference [A], is a consequence of the fact that if $H$ is a subgroup of $G$, then $K^{t}[G]$ is a free right or left $K^{t}[H]$-module (see also [P5, Lemmas 7.1.5 and 7.4.2]).

Lemma 7.1. Let $K^{t}[G]$ be a twisted group algebra with $G$ an arbitrary group. If $H$ is a subgroup of $G$, then $\mathcal{J} K^{t}[G] \cap K^{t}[H] \subseteq \mathcal{J} K^{t}[H]$. In particular, if $G$ is locally finite, then $\mathcal{J} K^{t}[G]$ is a nil ideal.

The next result, found in $[\mathrm{V}]$, follows from Nakayama's Lemma and Maschke's Theorem (see also [P5, Theorem 7.2.7]).

Lemma 7.2. Let $K^{t}[G]$ be given with $G$ an arbitrary group. If $N$ is a normal subgroup of $G$ of finite index $n$, then

$$
\left(\mathcal{J} K^{t}[G]\right)^{n} \subseteq \mathcal{J} K^{t}[N] \cdot K^{t}[G] \subseteq \mathcal{J} K^{t}[G] .
$$

Furthermore, if $n \neq 0$ in $K$, then $\mathcal{J} K^{t}[G]=\mathcal{J} K^{t}[N] \cdot K^{t}[G]$.

In particular, if char $K=p>0$ and $G / N$ is a finite $p^{\prime}$-group, then the latter equality holds. More generally, we have

Lemma 7.3. Let $K^{t}[G]$ be a twisted group algebra and let $N \triangleleft G$. If $G / N$ is a locally finite group, then $\mathcal{J} K^{t}[N] \cdot K^{t}[G] \subseteq \mathcal{J} K^{t}[G]$. Furthermore, if char $K=p>$ 0 and $G / N$ is a $p^{\prime}$-group, then $\mathcal{J} K^{t}[G]=\mathcal{J} K^{t}[N] \cdot K^{t}[G]$.

Proof. Since $G / N$ is locally finite, it follows that

$$
\mathcal{J} K^{t}[N] \cdot K^{t}[G]=\bigcup_{X} \mathcal{J} K^{t}[N] \cdot K^{t}[X]
$$

where the union is over all groups $X$ with $N \subseteq X \subseteq G$ and $|X: N|<\infty$. Moreover, by the preceding lemma, we have $\mathcal{J} K^{t}[N] \cdot K^{t}[X] \subseteq \mathcal{J} K^{t}[X]$ and, in particular, every element of $\mathcal{J} K^{t}[N] \cdot K^{t}[X]$ is quasi-regular. It follows that $\mathcal{J} K^{t}[N] \cdot K^{t}[G]$ is a quasi-regular ideal of $K^{t}[G]$ and therefore this ideal is contained in $\mathcal{J} K^{t}[G]$.

For the other direction, suppose that char $K=p>0$ and that $G / N$ is a $p^{\prime}$ group. If $\alpha \in \mathcal{J} K^{t}[G]$ then, since $G / N$ is locally finite, there exists a group $X$ as above with $\alpha \in K^{t}[X]$. But then $\alpha \in \mathcal{J} K^{t}[G] \cap K^{t}[X] \subseteq \mathcal{J} K^{t}[X]$, by Lemma 7.1, and hence, since $X / N$ is a $p^{\prime}$-group, it follows from Lemma 7.2 that $\alpha \in \mathcal{J} K^{t}[N] \cdot K^{t}[X] \subseteq \mathcal{J} K^{t}[N] \cdot K^{t}[G]$. 
In the remainder of this section, all groups considered will be locally finite and $K$ will denote a fixed field of characteristic $p>0$. Furthermore, we will assume that $K^{t}[G]$ is a given twisted group algebra of $G$ over $K$.

Lemma 7.4. If $A$ is locally subnormal in $G$, then $\mathcal{J} K^{t}[A] \subseteq \mathcal{J} K^{t}[G]$.

Proof. If $B$ is any finite subgroup of $G$ containing $A$, then $A \triangleleft \triangleleft B$ and hence $\mathcal{J} K^{t}[A] \subseteq \mathcal{J} K^{t}[B]$ by Lemma 7.2 and induction. In particular, $\mathcal{J} K^{t}[A] \cdot K^{t}[B]$ is a nil right ideal of $K^{t}[B]$ and hence $\mathcal{J} K^{t}[A] \cdot K^{t}[G]=\bigcup_{B} \mathcal{J} K^{t}[A] \cdot K^{t}[B]$ is a nil right ideal of $K^{t}[G]$.

If $H$ is a subgroup of $G$, then we define the twisted centralizer of $H$ in $G$ by

$$
\mathbb{C}_{G}^{t}(H)=\{g \in G \mid \bar{g} \bar{h}=\bar{h} \bar{g} \text { for all } h \in H\},
$$

and it is easy to see that $\mathbb{C}_{G}^{t}(H)$ is a subgroup of $G$ contained in $\mathbb{C}_{G}(H)$. Indeed, we have

Lemma 7.5. Let $K^{t}[G]$ be given and let $H \subseteq G$. Then $\mathbb{N}_{G}(H)$ acts on $K^{t}[H]$ by conjugation, and the kernel of this action is $\mathbb{C}_{G}^{t}(H)$. Furthermore, $\mathbb{N}_{G}(H) / \mathbb{C}_{G}(H)$ is isomorphic to a subgroup of $\operatorname{Aut}(H)$, and $\mathbb{C}_{G}(H) / \mathbb{C}_{G}^{t}(H)$ is isomorphic to a subgroup of the dual group $\operatorname{Hom}\left(H, K^{\bullet}\right)$. In particular, since $K$ has characteristic $p>0$, it follows that $\mathbb{C}_{G}(H) / \mathbb{C}_{G}^{t}(H)$ is an abelian $p^{\prime}$-group.

Proof. For each $g \in \mathbb{C}_{G}(H)$, define $\lambda_{g}: H \rightarrow K^{\bullet}$ by $\bar{g}^{-1} \bar{h} \bar{g}=\lambda_{g}(h) \bar{h}$ for all $h \in$ $H$. Then it is easy to see that $\lambda_{g} \in \operatorname{Hom}\left(H, K^{\bullet}\right)$ and that the map $\mathbb{C}_{G}(H) \rightarrow$ $\operatorname{Hom}\left(H, K^{\bullet}\right)$ given by $g \mapsto \lambda_{g}$ is a homomorphism. Since the kernel of this map is clearly $\mathbb{C}_{G}^{t}(H)$, the lemma is proved.

The next two lemmas are twisted versions of results from [DZ]. The proofs given here are somewhat easier than the original arguments because we assume that $G$ is locally finite.

Lemma 7.6. Let $H$ be an f.c. group and let $\alpha \in K^{t}[H]$ with $\alpha \notin \mathcal{J} K^{t}[H]$. If

$$
Y(\alpha)=\left\{y \in H \mid \alpha\left(1-\mu_{y} \bar{y}\right) \in \mathcal{J} K^{t}[H] \text { for some } \mu_{y} \in K^{\bullet}\right\},
$$

then $Y(\alpha)$ is a subgroup of $H$. Moreover, if $\mathbb{O}_{p}(H)$ is finite, then $Y(\alpha)$ is finite.

Proof. If $x, y \in Y=Y(\alpha)$, then

$$
\alpha\left(1-\mu_{x} \mu_{y}^{-1} \bar{x} \bar{y}^{-1}\right)=\left[\alpha\left(1-\mu_{x} \bar{x}\right)-\alpha\left(1-\mu_{y} \bar{y}\right)\right] \mu_{y}^{-1} \bar{y}^{-1} \in \mathcal{J} K^{t}[H],
$$

so $x y^{-1} \in Y$. Thus, since $1 \in Y$, we see that $Y$ is a subgroup of $G$.

Suppose now that $\mathbb{O}_{p}(H)$ is finite. Since $\alpha \notin \mathcal{J} K^{t}[H]$, it follows that $K^{t}[H] \alpha$ is not a nil left ideal, and hence there exists $\beta \in K^{t}[H]$ with $\beta \alpha$ not nilpotent. Furthermore, since $Y(\alpha) \subseteq Y(\beta \alpha)$, it suffices to prove that $Y(\beta \alpha)$ is finite, or equivalently, we can now assume that $\alpha$ is not nilpotent.

By assumption, $H$ is an f.c. group, and therefore there exists a finite normal subgroup $W$ of $H$ with $\alpha \in K^{t}[W]$. Furthermore, note that $C=\mathbb{C}_{H}^{t}(W)$ is a normal subgroup of $H$ of finite index by the preceding lemma, and of course $\mathbb{O}_{p}(C)$ is also finite. Since $X=Y \cap C$ is a normal subgroup of $Y$ of finite index, it clearly suffices to show that $X$ is finite.

To this end, let $x \in X$. Then $\alpha\left(1-\mu_{x} \bar{x}\right) \in \mathcal{J} K^{t}[H]$, so this element is nilpotent by Lemma 7.1, and therefore $\left[\alpha\left(1-\mu_{x} \bar{x}\right)\right]^{p^{n}}=0$ for some integer $n$. But $\bar{x}$ commutes with $\alpha$ since $x \in C$, so this yields

$$
0=\left[\alpha\left(1-\mu_{x} \bar{x}\right)\right]^{p^{n}}=\alpha^{p^{n}}\left(1-\mu_{x} \bar{x}\right)^{p^{n}}=\alpha^{p^{n}}\left(1-\mu_{x}^{p^{n}} \bar{x}^{p^{n}}\right) .
$$


In particular, since $\alpha$ is not nilpotent, we conclude that $x^{p^{n}} \in W$. In other words, $X /(X \cap W)$ is a $p$-group.

Finally, if $x \in X$ and $c \in C$, then since $\bar{c}$ centralizes $\alpha$, we have

$$
\alpha\left(1-\mu_{x} \bar{c}^{-1} \bar{x} \bar{c}\right)=\bar{c}^{-1} \alpha\left(1-\mu_{x} \bar{x}\right) \bar{c} \in \mathcal{J} K^{t}[H],
$$

so $c^{-1} x c \in X$. Thus $X \triangleleft C$ and hence $\mathbb{O}_{p}(X)$ is finite. Furthermore, note that $X \cap W$ is central in $X$ and therefore, since $X /(X \cap W)$ is a $p$-group, it follows that $X$ is locally nilpotent. Consequently, we must have $X=\mathbb{O}_{p}(X)(X \cap W)$, so $X$ is finite and the lemma is proved.

As usual, if $\alpha=\sum_{x \in G} a_{x} \bar{x} \in K^{t}[G]$, then the support of $\alpha$ is the finite subset of $G$ given by $\operatorname{supp} \alpha=\left\{x \in G \mid a_{x} \neq 0\right\}$. The next result is a crucial intersection theorem, namely a result which yields information about the intersection of an ideal $I \triangleleft K^{t}[G]$ with $K^{t}[D]$ for some $D \triangleleft G$.

Lemma 7.7. Let $F \subseteq S \subseteq D \subseteq G$ be normal subgroups of $G$ with $D=\mathbb{D}_{G}(F)$. Furthermore, suppose that $\mathbb{O}_{p}(G)$ is finite.

i. If $I \triangleleft K^{t}[G]$ and $I \supset \mathcal{J} K^{t}[S] \cdot K^{t}[G]$, then $I \cap K^{t}[D] \supset \mathcal{J} K^{t}[S] \cdot K^{t}[D]$.

ii. If $\mathcal{J} K^{t}[D]=\mathcal{J} K^{t}[S] \cdot K^{t}[D]$, then $\mathcal{J} K^{t}[G]=\mathcal{J} K^{t}[S] \cdot K^{t}[G]$.

Proof. (i) Since $I \supset \mathcal{J} K^{t}[S] \cdot K^{t}[G]$, we can choose $\alpha \in I$ with $\alpha \notin \mathcal{J} K^{t}[S] \cdot K^{t}[G]$ so that $\operatorname{supp} \alpha$ is contained in the minimal number, say $n$, of cosets of $S$ in $G$. Moreover, by multiplying $\alpha$ by a suitable element of $\bar{G}$, we can further assume that $1 \in \operatorname{supp} \alpha$. Thus we can write

$$
\alpha=\alpha_{1} \bar{g}_{1}+\alpha_{2} \bar{g}_{2}+\cdots+\alpha_{n} \bar{g}_{n}
$$

with $\alpha_{i} \in K^{t}[S]$ and with $1=g_{1}, g_{2}, \ldots, g_{n}$ in distinct cosets of $S$ in $G$. Now if $\alpha_{i} \in \mathcal{J} K^{t}[S]$ for some $i$, then $\alpha_{i} \bar{g}_{i} \in \mathcal{J} K^{t}[S] \cdot K^{t}[G] \subseteq I$ and therefore $\alpha-\alpha_{i} \bar{g}_{i}$ is an element of $I$, but not of $\mathcal{J} K^{t}[S] \cdot K^{t}[G]$, whose support is contained in less than $n$ cosets of $S$, a contradiction. Thus $\alpha_{i} \notin \mathcal{J} K^{t}[S]$ and, in particular, the right ideal $\alpha_{i} K^{t}[S]$ is not nil. Choose $\beta_{i} \in K^{t}[S]$ so that $\alpha_{i} \beta_{i}$ is not nilpotent.

Let $W$ be the subgroup of $S$ generated by $F$ and the supports of all $\alpha_{i}$ and $\beta_{i}$. Then clearly $|W: F|<\infty$, so since $W \subseteq S \subseteq \mathbb{D}_{G}(F)$, we see that $W$ is an f.c. group. In particular, there exists a finite normal subgroup $N$ of $W$ which contains the supports of all $\alpha_{i}$, and we set $L=\mathbb{C}_{F}^{t}(N)$. Of course, $|F: L|<\infty$ by Lemma 7.5 and $\bar{L}$ centralizes each $\alpha_{i}$. Note that we do not assert that either $W$ or $L$ is normal in $G$. Now let $x \in L$. Then $\alpha-\alpha^{\bar{x}} \in I$ and, since $g_{1}=1$ and $\bar{x}$ centralizes all $\alpha_{i}$, we have

$$
\alpha-\alpha^{\bar{x}}=\sum_{i=2}^{n} \alpha_{i}\left(1-\bar{g}_{i}^{\bar{x}} \bar{g}_{i}^{-1}\right) \bar{g}_{i} \in I .
$$

Note that $x \in L \subseteq F \triangleleft G$, so $g_{i}^{x} g_{i}^{-1} \in F \subseteq S$. In particular, we see that $\alpha-\alpha^{\bar{x}}$ has support contained in less than $n$ cosets of $S$. Thus, by the minimality of $n$, we have $\alpha-\alpha^{\bar{x}} \in \mathcal{J} K^{t}[S] \cdot K^{t}[G]$ and hence $\alpha_{i}\left(1-\bar{g}_{i}^{\bar{x}} \bar{g}_{i}^{-1}\right) \in \mathcal{J} K^{t}[S]$ for all $i>1$.

We now wish to apply the preceding lemma to the f.c. group $W$. To this end, note that $\alpha_{i} \beta_{i} \in \alpha_{i} K^{t}[W]$, so the latter is not a nil right ideal and hence $\alpha_{i} \notin \mathcal{J} K^{t}[W]$ by Lemma 7.1. Furthermore, $\mathbb{O}_{p}(F) \subseteq \mathbb{O}_{p}(G)$, so $\mathbb{O}_{p}(F)$ is finite and hence so is $\mathbb{O}_{p}(W)$. Finally, for all $i>1$,

$$
\alpha_{i}\left(1-\bar{g}_{i}^{\bar{x}} \bar{g}_{i}^{-1}\right) \in \mathcal{J} K^{t}[S] \cap K^{t}[W] \subseteq \mathcal{J} K^{t}[W] .
$$


Thus, for fixed $i>1$, Lemma 7.6 applies to $\alpha_{i}$ and consequently $g_{i}^{x} g_{i}^{-1} \in F \subseteq W$ is contained in the finite group $Y\left(\alpha_{i}\right)$. Indeed, this holds for all $x \in L$, so we conclude that $\left|L: \mathbb{C}_{L}\left(g_{i}\right)\right|<\infty$. Since the inequality $\left|L: \mathbb{C}_{L}\left(g_{i}\right)\right|<\infty$ is trivially also true for $g_{1}=1$ and since $|F: L|<\infty$, it follows that $g_{i} \in \mathbb{D}_{G}(F)=D$ for all $i$. In other words, $\alpha \in I \cap K^{t}[D]$. But $\alpha \notin \mathcal{J} K^{t}[S] \cdot K^{t}[D]$ since $\alpha_{i} \notin \mathcal{J} K^{t}[S]$, so $I \cap K^{t}[D] \supset \mathcal{J} K^{t}[S] \cdot K^{t}[D]$ and part (i) is proved.

(ii) Here we let $I=\mathcal{J} K^{t}[G]$ so that $I \supseteq \mathcal{J} K^{t}[S] \cdot K^{t}[G]$ by Lemma 7.3. Furthermore,

$$
I \cap K^{t}[D] \subseteq \mathcal{J} K^{t}[D]=\mathcal{J} K^{t}[S] \cdot K^{t}[D],
$$

by Lemma 7.1 and the hypothesis. Thus $I \cap K^{t}[D]=\mathcal{J} K^{t}[S] \cdot K^{t}[D]$ and therefore part (i) implies that $I=\mathcal{J} K^{t}[S] \cdot K^{t}[G]$.

We will only require the first part of the above result, but the second is certainly indicative of its power.

Next, we need some elementary properties of $K^{t}[G]$-modules when $G$ is finite. For example, part (ii) below is a special case of the Mackey decomposition, while parts (i) and (iii) are aspects of Frobenius reciprocity which remain valid in characteristic $p>0$. Note that, if $H$ is a subgroup of $G$, then $V_{H}$ denotes the restriction of the $K^{t}[G]$-module $V$ to $K^{t}[H]$. Furthermore, if $W$ is a $K^{t}[H]$-module, then we write $W^{G}$ for the induced $K^{t}[G]$-module $W^{G}=W \otimes_{K^{t}[H]} K^{t}[G]$.

Lemma 7.8. Let $A$ and $B$ be subgroups of the finite group $G$, let $W$ be an irreducible $K^{t}[A]$-module and let $V$ be an irreducible $K^{t}[G]$-module.

i. $V$ is a composition factor of $\left(V_{B}\right)^{G}$.

ii. $\left(W^{G}\right)_{B}$ contains an isomorphic copy of $\left(W_{A \cap B}\right)^{B}$.

iii. If $A \triangleleft \triangleleft G$ and $U$ is a composition factor of $W^{G}$, then $W$ is isomorphic to a submodule of $U_{A}$.

Proof. (i) If $x_{1}, x_{2}, \ldots, x_{n}$ is a right transversal for $B$ in $G$, then it is easy to see that the map $\left(V_{B}\right)^{G} \rightarrow V$ given by $\sum_{i} v_{i} \otimes \bar{x}_{i} \rightarrow \sum_{i} v_{i} \bar{x}_{i}$ is a $K^{t}[G]$-module epimorphism.

(ii) If $y_{1}, y_{2}, \ldots, y_{m}$ is a right transversal for $A \cap B$ in $B$, then these elements are also part of a transversal for $A$ in $G$. Thus $Y=\sum_{i} W \otimes \bar{y}_{i}$ is a subspace of $W^{G}$ which is easily seen to be a $K^{t}[B]$-submodule. Furthermore, as a $K^{t}[B]$-module, $Y$ is clearly determined by $W_{A \cap B}$, and hence

$$
\left(W^{G}\right)_{B} \supseteq Y=\sum_{i} W_{A \cap B} \otimes \bar{y}_{i} \cong\left(W_{A \cap B}\right)^{B} .
$$

(iii) Suppose first that $A \triangleleft G$ and let $z_{1}, z_{2}, \ldots, z_{k}$ be a transversal for $A$ in $G$. Then $W^{G}=\sum_{i} W \otimes \bar{z}_{i}$ and each $W \otimes \bar{z}_{i}$ is an irreducible $K^{t}[A]$-submodule of $\left(W^{G}\right)_{A}$. In particular, if $U$ is a $K^{t}[G]$-composition factor of $W^{G}$, then $U_{A}$ is completely reducible and contains a submodule isomorphic to $W \otimes \bar{z}_{i}$ for some $i$. But $U=U \bar{z}_{i}^{-1}$, so we see that $U_{A}$ also contains a submodule isomorphic to $W$.

Finally, let $A \triangleleft \triangleleft G$ and proceed by induction along a subnormal chain joining $A$ to $G$. To this end, let $A_{1} \supset A$ with $A \triangleleft A_{1}$ and $A_{1} \triangleleft \triangleleft G$. If $U$ is a composition factor of $W^{G}$, then since $W^{G}=\left(W^{A_{1}}\right)^{G}$, we see that $U$ is isomorphic to a composition factor of $W_{1}^{G}$ for some $K^{t}\left[A_{1}\right]$-module $W_{1}$ which is a composition factor of $W^{A_{1}}$. By induction, $U_{A_{1}}$ contains an isomorphic copy of $W_{1}$, and since $A \triangleleft A_{1}$, we see that $\left(W_{1}\right)_{A}$ contains an isomorphic copy of $W$. Thus $U_{A} \supseteq W^{\prime} \cong W$, and the lemma is proved. 
As a consequence, we have

Lemma 7.9. Let $A$ and $B$ be subgroups of the finite group $G$ and suppose that $A \triangleleft \triangleleft G$. Furthermore, let $W$ be an irreducible $K^{t}[A]$-module, let $V$ be an irreducible $K^{t}[B]$-module, and suppose that $V_{A \cap B}$ is isomorphic to a submodule of $W_{A \cap B}$. Then there exists an irreducible $K^{t}[G]$-module $U$ such that $W$ is isomorphic to a submodule of $U_{A}$ and $V$ is a composition factor of $U_{B}$.

Proof. By parts (i) and (ii) of the preceding lemma, we have (up to isomorphism)

$$
\left(W^{G}\right)_{B} \supseteq\left(W_{A \cap B}\right)^{B} \supseteq\left(V_{A \cap B}\right)^{B}
$$

and the latter module has $V$ as a composition factor. Thus, there exists a composition factor $U$ of $W^{G}$ such that $V$ occurs as a composition factor of $U_{B}$. Finally, since $A \triangleleft \triangleleft G$, Lemma 7.8(iii) implies that $W$ is isomorphic to a submodule of $U_{A}$.

We note that the above result does not remain valid when the assumption that $A$ is subnormal in $G$ is dropped. For example, let $G$ be the nonabelian group of order 6 and let $K^{t}[G]=K[G]$ be the ordinary group algebra with char $K=p=3$. If $A$ and $B$ are two distinct subgroups of order 2 and if $W$ is the principal $K[A]$-module and $V$ is the unique nonprincipal $K[B]$-module, then it is clear that no appropriate $K[G]$-module $U$ can exist.

Finally, if $D$ is any subgroup of the locally finite group $G$, then there is a natural $K^{t}[D]$-bimodule projection map $\pi_{D}: K^{t}[G] \rightarrow K^{t}[D]$ given by

$$
\pi_{D}: \sum_{x \in G} a_{x} \bar{x} \mapsto \sum_{x \in D} a_{x} \bar{x} .
$$

In other words, $\pi_{D}$ is the linear extension of the map $\bar{G} \rightarrow \bar{D} \cup\{0\}$ which is the identity on $\bar{D}$ and zero on $\bar{G} \backslash \bar{D}$. The following is the twisted version of [P5, Lemmas 1.5(ii) and 1.6], and the proofs are identical.

Lemma 7.10. If $I \triangleleft K^{t}[G]$ and $D \triangleleft G$, then $\pi_{D}(I)$ is a $G$-stable ideal of $K^{t}[D]$ with $I \subseteq \pi_{D}(I) \cdot K^{t}[D]$. Furthermore, $D$ controls $I$ if and only if $\pi_{D}(I) \subseteq I$, and when this occurs, we have $I \cap K^{t}[D]=\pi_{D}(I)$.

\section{Finite Extensions}

In this section, we study the ideal $J=\mathcal{J} K^{t}\left[\mathbb{S}^{p}(G)\right] \cdot K^{t}[G]$ under the assumption that $\mathbb{O}_{p}(G)$ is finite. Our goal is to show that $J$ is a semiprime ideal and to decide when it is prime. As a consequence, we show that the validity of the main theorem can be lifted from a normal subgroup of finite index to the full group. The arguments here are quite close to those of reference [P4], where similar results were obtained for ordinary group algebras. Indeed, only a few minor changes are required in the proofs.

As usual, we let $K^{t}[G]$ denote a twisted group algebra of the locally finite group $G$ over the field $K$ of characteristic $p>0$. Also, if $N$ is a subgroup of $G$, then $\pi_{N}: K^{t}[G] \rightarrow K^{t}[N]$ denotes the natural projection map. Recall that $\Delta(G)$ is the f.c. center of $G$ which is defined by

$$
\Delta(G)=\left\{g \in G|| G: \mathbb{C}_{G}(g) \mid<\infty\right\}
$$

and that, in the context of locally finite groups, $\Delta(G)$ is the join of all the finite normal subgroups of $G$. 
Lemma 8.1. Let $N$ be a normal subgroup of $G$ with $N=\mathbb{S}(N)$, and let $\alpha$ and $\beta$ be elements of $K^{t}[G]$. If $\alpha \beta^{\bar{g}} \in \mathcal{J} K^{t}[N] \cdot K^{t}[G]$ for all $g \in G$, then either $\pi_{N}(\alpha) \pi_{N}(\beta) \in \mathcal{J} K^{t}[N]$, or there exists $x \in \operatorname{supp} \beta$ with $1 \neq x N \in \Delta(G / N)$.

Proof. Write $\alpha=\alpha_{0}+\alpha_{1}$ and $\beta=\beta_{0}+\beta_{1}$, where $\alpha_{0}=\pi_{N}(\alpha)$ and $\beta_{0}=\pi_{N}(\beta)$. Furthermore, let us suppose that $\alpha_{0} \beta_{0} \notin \mathcal{J} K^{t}[N]$. Since $N=\mathbb{S}(N)$, it follows from Lemma 4.1(i) that there exists $A \operatorname{lsn} N$ with $\alpha, \beta \in K^{t}[A]$, and by Lemma 7.4, we have $\alpha_{0} \beta_{0} \notin \mathcal{J} K^{t}[A]$. Set $D=A \cap \Delta(G)$ and let $L=\mathbb{C}_{G}^{t}(D)$. Since $D$ is a finite subgroup of $\Delta(G)$, it is clear that $\left|G: \mathbb{C}_{G}(D)\right|<\infty$ and hence, by Lemma 7.5, we have $|G: L|<\infty$.

Let $a_{i}$ and $a_{j}$ be elements of $A \backslash D$. If $a_{i}$ and $a_{j}$ are conjugate under the action of $L$, choose $g_{i, j} \in L$ with $g_{i, j}^{-1} a_{i} g_{i, j}=a_{j}$. Similarly, let $x_{i} \in \operatorname{supp} \beta_{1}$ and $y_{j} \in \operatorname{supp} \alpha_{1}$. If $x_{i}$ and $y_{j}^{-1}$ are conjugate modulo $N$ under the action of $L$, choose $h_{i, j} \in L$ with $h_{i, j}^{-1} x_{i} h_{i, j} \in y_{j}^{-1} N$. We claim that

$$
L=\bigcup_{i, j} \mathbb{C}_{L}\left(a_{i}\right) g_{i, j} \cup \bigcup_{i, j} \mathbb{C}_{L}\left(x_{i} N\right) h_{i, j},
$$

where $\mathbb{C}_{L}\left(x_{i} N\right)$ is the centralizer in $L$ of the element $x_{i} N \in G / N$.

To this end, let $g \in L$, so that clearly $A \cap A^{g} \supseteq D$. If $A \cap A^{g} \supset D$, then there exist $a_{i}, a_{j} \in A \backslash D$ with $a_{i}^{g}=a_{j}$. In particular, this means that $a_{i}$ and $a_{j}$ are conjugate under the action of $L$, so the definition of $g_{i, j}$ implies that $g \in \mathbb{C}_{L}\left(a_{i}\right) g_{i, j}$. Thus, we may suppose that $A \cap A^{g}=D$ and we set $E=\left\langle A, A^{g}\right\rangle$. Since $A \operatorname{lsn} N$, it follows that $A, A^{g} \triangleleft \triangleleft E$.

Now $\alpha_{0} \beta_{0} \notin \mathcal{J} K^{t}[A]$, so there exists an irreducible representation $\mathcal{X}$ of $K^{t}[A]$ with $\mathcal{X}\left(\alpha_{0} \beta_{0}\right) \neq 0$. In particular, $\mathcal{X}\left(\alpha_{0}\right) \neq 0$ and $\mathcal{X}\left(\beta_{0}\right) \neq 0$. Furthermore, $\mathcal{X}^{\bar{g}}$ is an irreducible representation of $K^{t}[B]$ where $B=A^{g}$ and $\mathcal{X}^{\bar{g}}\left(\beta_{0}^{\bar{g}}\right)=\mathcal{X}\left(\beta_{0}\right) \neq 0$. Since $g \in \mathbb{C}_{G}^{t}(D)$, it is clear that $\mathcal{X}$ and $\mathcal{X}^{\bar{g}}$ agree on $K^{t}[A \cap B]=K^{t}[D]$. Thus, since $A \triangleleft \triangleleft E$, it follows from Lemma 7.9 that there exists an irreducible representation $\mathcal{Y}$ of $K^{t}[E]$ such that $\mathcal{X}$ is a constituent of the restriction of $\mathcal{Y}$ to $K^{t}[A]$, and $\mathcal{X}^{\bar{g}}$ is a constituent of the restriction of $\mathcal{Y}$ to $K^{t}[B]$. Consequently, we have $\mathcal{Y}\left(\alpha_{0}\right) \neq 0$ and $\mathcal{Y}\left(\beta_{0}^{\bar{g}}\right) \neq 0$. But $\mathcal{Y}\left(K^{t}[E]\right)$ is a prime ring, so $\mathcal{Y}\left(\alpha_{0} K^{t}[E] \beta_{0}^{\bar{g}}\right) \neq 0$ and hence there exists $e \in E$ with $\mathcal{Y}\left(\alpha_{0} \bar{e}^{-1} \beta_{0}^{\bar{g}}\right) \neq 0$. In other words, $\alpha_{0} \bar{e}^{-1} \beta_{0}^{\bar{g}} \notin \mathcal{J} K^{t}[E]$, so $\alpha_{0} \bar{e}^{-1} \beta_{0}^{\bar{g}} \notin \mathcal{J} K^{t}[N]$, by Lemma 7.1, and hence $\alpha_{0} \beta_{0}^{\bar{g} \bar{e}}=\alpha_{0} \bar{e}^{-1} \beta_{0}^{\bar{g}} \bar{e} \notin \mathcal{J} K^{t}[N]$.

By assumption, $\alpha \beta^{\bar{g} \bar{e}} \in \mathcal{J} K^{t}[N] \cdot K^{t}[G]$. Thus since $\alpha_{0} \beta_{0}^{\bar{g} \bar{e}} \notin \mathcal{J} K^{t}[N]$, it follows that the support of $\alpha_{1} \beta_{1}^{\bar{g} \bar{e}}$ must contain an element of $N$. In particular, there exist $y_{j} \in \operatorname{supp} \alpha_{1}$ and $x_{i} \in \operatorname{supp} \beta_{1}$ with $y_{j} x_{i}^{g e} \in N$. Viewing this equation modulo $N$ and using the fact that $e \in E \subseteq N$, we see that $x_{i}^{g} \in y_{j}^{-1} N$, and hence the definition of $h_{i, j}$ implies that $g \in \mathbb{C}_{L}\left(x_{i} N\right) h_{i, j}$.

We have therefore shown that

$$
L=\bigcup_{i, j} \mathbb{C}_{L}\left(a_{i}\right) g_{i, j} \cup \bigcup_{i, j} \mathbb{C}_{L}\left(x_{i} N\right) h_{i, j}
$$

so $L$ is a finite union of cosets of the centralizer subgroups, and [P5, Lemma 4.2.1] implies that one of these subgroups has finite index in $L$ and hence in $G$. Now, if $\left|G: \mathbb{C}_{L}\left(a_{i}\right)\right|<\infty$, then $a_{i} \in A \cap \Delta(G)=D$, a contradiction. Thus, we must have $\left|G: \mathbb{C}_{L}\left(x_{i} N\right)\right|<\infty$ and therefore $x_{i} N \in \Delta(G / N)$. But $x_{i} \in \operatorname{supp} \beta_{1}=(\operatorname{supp} \beta) \backslash N$, so $x_{i} \notin N$ and the lemma is proved.

The coset counting argument above exhibits the flavor of the group algebra technique known as the Delta method. In a similar manner, we obtain 
Lemma 8.2. Let $N \triangleleft G$ with $N=\mathbb{S}(N)$ and let $\alpha, \beta \in K^{t}[N]$ satisfy $\alpha \beta^{\bar{g}} \in$ $\mathcal{J} K^{t}[N]$ for all $g \in G$. If $N \cap \Delta(G)=1$, then either $\alpha \in \mathcal{J} K^{t}[N]$ or $\beta \in \mathcal{J} K^{t}[N]$.

Proof. We assume, by way of contradiction, that $\alpha \notin \mathcal{J} K^{t}[N]$ and $\beta \notin \mathcal{J} K^{t}[N]$. Since $N=\mathbb{S}(N)$, there exists $A$ lsn $N$ with $\alpha, \beta \in K^{t}[A]$, and Lemma 7.4 implies that $\alpha, \beta \notin \mathcal{J} K^{t}[A]$. In particular, we can let $\mathcal{X}$ and $\mathcal{Y}$ be irreducible representations of $K^{t}[A]$ with $\mathcal{X}(\alpha) \neq 0$ and $\mathcal{Y}(\beta) \neq 0$.

Suppose first that there exists $g \in G$ with $A \cap A^{g}=1$. If $E=\left\langle A, A^{g}\right\rangle$, then $A$ and $A^{g}$ are subnormal in $E$. Furthermore, $\mathcal{X}$ is an irreducible representation of $K^{t}[A]$ and $\mathcal{Y}^{\bar{g}}$ is an irreducible representation of $K^{t}\left[A^{g}\right]$. Since $A \cap A^{g}=1$, one of the restrictions $\mathcal{X}_{A \cap A^{g}}$ and $\left(\mathcal{Y}^{\bar{g}}\right)_{A \cap A^{g}}$ must contain an isomorphic copy of the other. Hence, by Lemma 7.9, there exists an irreducible representation $\mathcal{Z}$ of $K^{t}[E]$ such that $\mathcal{X}$ is a constituent of $\mathcal{Z}_{A}$ and $\mathcal{Y}^{\bar{g}}$ is a constituent of $\mathcal{Z}_{A^{g}}$. But $\mathcal{Y}^{\bar{g}}\left(\beta^{\bar{g}}\right)=\mathcal{Y}(\beta) \neq 0$, and therefore $\mathcal{Z}(\alpha) \neq 0$ and $\mathcal{Z}\left(\beta^{\bar{g}}\right) \neq 0$. In particular, since $\mathcal{Z}\left(K^{t}[E]\right)$ is a prime ring, this yields $\mathcal{Z}\left(\alpha K^{t}[E] \beta^{\bar{g}}\right) \neq 0$ and thus there exists $e \in E$ with $\mathcal{Z}\left(\alpha \bar{e}^{-1} \beta^{\bar{g}}\right) \neq 0$. Consequently, $\alpha \beta^{\bar{g} \bar{e}} \notin \mathcal{J} K^{t}[E]$, so Lemma 7.1 implies that $\alpha \beta^{\bar{g} \bar{e}} \notin \mathcal{J} K^{t}[N]$, contrary to the hypothesis. In other words, we must have $A \cap A^{g} \neq 1$ for all $g \in G$.

If $a_{i}, a_{j} \in A \backslash 1$ are conjugate in $G$, choose $g_{i, j} \in G$ with $g_{i, j}^{-1} a_{i} g_{i, j}=a_{j}$. Now let $g \in G$. Since $A \cap A^{g} \neq 1$, there exist $a_{i}, a_{j} \in A \backslash 1$ with $a_{i}^{g}=a_{j}$. Thus $a_{i}$ and $a_{j}$ are conjugate in $G$, so the definition of $g_{i, j}$ implies that $g \in \mathbb{C}_{G}\left(a_{i}\right) g_{i, j}$. In other words, we have shown that $G$ is the finite union

$$
G=\bigcup_{i, j} \mathbb{C}_{G}\left(a_{i}\right) g_{i, j},
$$

so by [P5, Lemma 4.2.1], we have $\left|G: \mathbb{C}_{G}\left(a_{i}\right)\right|<\infty$ for some $i$. But then $1 \neq a_{i} \in$ $A \cap \Delta(G) \subseteq N \cap \Delta(G)$, contradicting the hypothesis of the lemma.

We can now obtain the twisted version of [P4, Theorem 5.2]. Since our main theorem asserts that $\mathcal{J} K^{t}[G]$ is equal to $\mathcal{J} K^{t}\left[\mathbb{S}^{p}(G)\right] \cdot K^{t}[G]$, it is not surprising that the latter is a semiprime ideal. However, this fact is a necessary step in the proof of Theorem 1.2.

Proposition 8.3. Let $K^{t}[G]$ be a twisted group algebra of the locally finite group $G$ over the field $K$ of characteristic $p>0$. If $\mathbb{O}_{p}(G)$ is finite, then $\mathcal{J} K^{t}\left[\mathbb{S}^{p}(G)\right] \cdot K^{t}[G]$ is a semiprime ideal.

Proof. Since $\mathbb{O}_{p}(G)$ is finite, it follows that $\mathbb{O}_{p}(N)$ is finite for all normal subgroups $N$ of $G$. Now set $S=\mathbb{S}^{p}(G)$ and let $F=\mathbb{F}^{*}(S)$. If $D=\mathbb{D}_{G}(F)$, then Lemma 4.4 applied to $S$ shows that $F \subseteq S \subseteq D$ is a chain of normal subgroups of $G$. For convenience, write $J=\mathcal{J} K^{t}[S] \cdot K^{t}[G]$ and suppose that $I$ is an ideal of $K^{t}[G]$ with $I \supseteq J$ and $I^{2} \subseteq J$. The goal is to show that $I=J$. To this end, let $\tilde{I}=I \cap K^{t}[D]$. Then we know that $\tilde{I}$ is a $G$-stable ideal of $K^{t}[D]$ with $\tilde{I} \supseteq \mathcal{J} K^{t}[S] \cdot K^{t}[D]$ and $\tilde{I}^{2} \subseteq J \cap K^{t}[D]=\mathcal{J} K^{t}[S] \cdot K^{t}[D]$.

Fix $\alpha$ in $\tilde{I}$ and let $N / S \subseteq D / S$ be a finite normal subgroup of $G / S$ containing all those elements of $(\operatorname{supp} \alpha) S$ which are in $\Delta(G / S)$. Thus $N \triangleleft G$ and $|N: S|<\infty$. Furthermore, if $x \in \operatorname{supp} \alpha$ has only finitely many $G$-conjugates modulo $N$, then $x$ has only finitely many $G$-conjugates modulo $S$, and therefore $x \in N$ by the choice of $N$. We note that $N=S H$ for some finite subgroup $H$ of $G$, and since $S \triangleleft N$ with $S=\mathbb{S}(S)$, we have $S \subseteq \mathbb{S}(N)$ and hence $N=\mathbb{S}(N) H$. Furthermore, Lemma 4.3(iii) implies that $\mathbb{F}^{*}(N) \cap S=\mathbb{F}^{*}(S)=F$. Thus $\left|\mathbb{F}^{*}(N): F\right|<\infty$ and consequently 
$\left|\mathbb{F}^{*}(N): \mathbb{C}_{\mathbb{F}^{*}(N)}(H)\right|<\infty$ since $H \subseteq D=\mathbb{D}_{G}(F)$. As we observed, $\mathbb{O}_{p}(N)$ is finite, so we conclude from Proposition 5.3 that $N=\mathbb{S}(N)$.

Now fix $\gamma \in K^{t}[N]$ and let $g \in G$. Then $\alpha \gamma \in \tilde{I}$ and hence so is $(\alpha \gamma)^{\bar{g}}$. Thus,

$$
(\alpha \gamma)(\alpha \gamma)^{\bar{g}} \in \tilde{I}^{2} \subseteq \mathcal{J} K^{t}[S] \cdot K^{t}[D] \subseteq \mathcal{J} K^{t}[N] \cdot K^{t}[G]
$$

by Lemma 7.3. Furthermore, since $\gamma \in K^{t}[N]$, it follows that $\operatorname{supp} \alpha \gamma \subseteq(\operatorname{supp} \alpha) N$ and therefore if $y \in \operatorname{supp} \alpha \gamma$ with $y N \in \Delta(G / N)$, then $y \in N$. Lemma 8.1 applied to the element $\alpha \gamma$ now shows that $\left(\pi_{N}(\alpha \gamma)\right)^{2} \in \mathcal{J} K^{t}[N]$ and hence $\pi_{N}(\alpha \gamma)$ is nilpotent by Lemma 7.1. Of course, $\gamma \in K^{t}[N]$, so we have $\pi_{N}(\alpha \gamma)=\pi_{N}(\alpha) \gamma$. Thus, since $\gamma \in K^{t}[N]$ is arbitrary, we conclude that $\pi_{N}(\alpha) K^{t}[N]$ is a nil right ideal of $K^{t}[N]$, and hence $\pi_{N}(\alpha) \in \mathcal{J} K^{t}[N]$.

Note that $\mathbb{S}^{p}(G)=S \subseteq N=\mathbb{S}(N) \subseteq \mathbb{S}(G)$, so $N / S$ is a finite $p^{\prime}$-group and hence $\mathcal{J} K^{t}[N]=\mathcal{J} K^{t}[S] \cdot K^{t}[N]$ by Lemma 7.2. In particular, since $\pi_{N}(\alpha) \in \mathcal{J} K^{t}[N]$, we conclude that

$$
\pi_{S}(\alpha)=\pi_{S}\left(\pi_{N}(\alpha)\right) \subseteq \pi_{S}\left(\mathcal{J} K^{t}[S] \cdot K^{t}[N]\right)=\mathcal{J} K^{t}[S] .
$$

Thus $\pi_{S}(\tilde{I}) \subseteq \mathcal{J} K^{t}[S]$, and hence

$$
I \cap K^{t}[D]=\tilde{I} \subseteq \pi_{S}(\tilde{I}) \cdot K^{t}[D] \subseteq \mathcal{J} K^{t}[S] \cdot K^{t}[D],
$$

by Lemma 7.10. Since $I \supseteq J=\mathcal{J} K^{t}[S] \cdot K^{t}[G]$, it now follows from Lemma 7.7(i) that $I=\mathcal{J} K^{t}[S] \cdot K^{t}[G]$, as required.

In a similar manner, we prove the twisted analog of [P4, Theorem 5.3].

Proposition 8.4. Let $K^{t}[G]$ be a twisted group algebra of the locally finite group $G$ over the field $K$ of characteristic $p>0$. If $\mathbb{O}_{p}(G)=1$ and $\Delta(G)=1$, then $\mathcal{J} K^{t}\left[\mathbb{S}^{p}(G)\right] \cdot K^{t}[G]$ is a prime ideal.

Proof. Since $\mathbb{O}_{p}(G)=1$, it follows that $\mathbb{O}_{p}(N)=1$ for all normal subgroups $N$ of $G$. Now set $S=\mathbb{S}^{p}(G)$ and let $F=\mathbb{F}^{*}(S)$. If $D=\mathbb{D}_{G}(F)$, then Lemma 4.4 applied to $S$ shows that $F \subseteq S \subseteq D$ is a chain of normal subgroups of $G$. For convenience, write $J=\mathcal{J} K^{t}[S] \cdot K^{t}[G]$ and let us assume that $J$ is not a prime ideal of $K^{t}[G]$. Thus, there exist ideals $I_{1}$ and $I_{2}$ of $K^{t}[G]$ properly containing $J$ with $I_{1} I_{2} \subseteq J$. By Lemma 7.7(i), we have $\tilde{I}_{i}=I_{i} \cap K^{t}[D] \supset \mathcal{J} K^{t}[S] \cdot K^{t}[D]$ for $i=1$, 2. Of course, $\tilde{I}_{1} \tilde{I}_{2} \subseteq J \cap K^{t}[D]=\mathcal{J} K^{t}[S] \cdot K^{t}[D]$.

Now $\tilde{I}_{i} \subseteq \pi_{S}\left(\tilde{I}_{i}\right) \cdot K^{t}[D]$ by Lemma 7.10 , so $\pi_{S}\left(\tilde{I}_{i}\right)$ must be strictly larger than $\mathcal{J} K^{t}[S]$. Therefore, we can choose $\alpha_{i} \in \tilde{I}_{i}$ with $\pi_{S}\left(\alpha_{i}\right) \notin \mathcal{J} K^{t}[S]$. Let $N / S \subseteq D / S$ be a finite normal subgroup of $G / S$ containing all those elements of $\left(\operatorname{supp} \alpha_{2}\right) S$ which are in $\Delta(G / S)$. Then $N \triangleleft G$ and $|N: S|<\infty$. Furthermore, if $x \in \operatorname{supp} \alpha_{2}$ has only finitely many $G$-conjugates modulo $N$, then $x$ has only finitely many $G$ conjugates modulo $S$, and therefore $x \in N$ by the choice of $N$.

Note that $N=S H$ for some finite subgroup $H$ of $G$, and since $S \triangleleft N$ with $S=\mathbb{S}(S)$, we have $S \subseteq \mathbb{S}(N)$ and hence $N=\mathbb{S}(N) H$. Furthermore, Lemma 4.3(iii) implies that $\mathbb{F}^{*}(N) \cap S=\mathbb{F}^{*}(S)=F$. Thus $\left|\mathbb{F}^{*}(N): F\right|<\infty$ and, since $H \subseteq D=$ $\mathbb{D}_{G}(F)$, we have $\left|\mathbb{F}^{*}(N): \mathbb{C}_{\mathbb{F}^{*}(N)}(H)\right|<\infty$. Since $\mathbb{O}_{p}(N)=1$, Proposition 5.3 now implies that $N=\mathbb{S}(N)$. In addition, $\mathbb{S}^{p}(G)=S \subseteq N=\mathbb{S}(N) \subseteq \mathbb{S}(G)$, so $N / S$ is a finite $p^{\prime}$-group and hence $\mathcal{J} K^{t}[N]=\mathcal{J} K^{t}[S] \cdot K^{t}[N]$ by Lemma 7.2. In particular, since $\pi_{S}\left(\pi_{N}\left(\alpha_{i}\right)\right)=\pi_{S}\left(\alpha_{i}\right) \notin \mathcal{J} K^{t}[S]$, it follows that $\pi_{N}\left(\alpha_{i}\right) \notin \mathcal{J} K^{t}[N]$ for $i=1,2$.

Fix $g \in G$. Then, for all $y \in G$ we have $\alpha_{2}^{\bar{g} \bar{y}} \in \tilde{I}_{2}$, and hence

$$
\alpha_{1} \alpha_{2}^{\bar{g} \bar{y}} \in \tilde{I}_{1} \tilde{I}_{2} \subseteq \mathcal{J} K^{t}[S] \cdot K^{t}[D] \subseteq \mathcal{J} K^{t}[N] \cdot K^{t}[G]
$$


by Lemma 7.2. Furthermore, $\operatorname{supp} \alpha_{2}^{\bar{g}}=\left(\operatorname{supp} \alpha_{2}\right)^{g}$, so any element of $\operatorname{supp} \alpha_{2}^{\bar{g}}$ having only finitely many $G$-conjugates modulo $N$ is contained in $N$. In particular, Lemma 8.1 applies and we have $\pi_{N}\left(\alpha_{1}\right) \pi_{N}\left(\alpha_{2}^{\bar{g}}\right) \in \mathcal{J} K^{t}[N]$.

We have therefore shown that $\pi_{N}\left(\alpha_{i}\right) \notin \mathcal{J} K^{t}[N]$, but that $\pi_{N}\left(\alpha_{1}\right) \pi_{N}\left(\alpha_{2}^{\bar{g}}\right) \in$ $\mathcal{J} K^{t}[N]$ for all $g \in G$. Since $N=\mathbb{S}(N)$ and $\mathbb{O}_{p}(N)=1$, we deduce from Lemma 8.2 that $N \cap \Delta(G) \neq 1$. In particular, $\Delta(G) \neq 1$ contrary to the hypothesis, and the result follows.

It is clear that some assumption like $\Delta(G)=1$ is needed to prove the primeness of $J=\mathcal{J} K^{t}\left[\mathbb{S}^{p}(G)\right] \cdot K^{t}[G]$. For example, take $G$ to be a finite group with $G=G^{p}$ and $\mathbb{O}_{p}(G)=1$. Then $J=\mathcal{J} K^{t}[G]$ and $J$ will not be a prime ideal when $K^{t}[G]$ has at least two distinct irreducible representations.

We close this section with a crucial consequence of Proposition 8.3. It handles those aspects of the proof of the main theorem which involve finite extensions.

Proposition 8.5. Let $K^{t}[G]$ be a characteristic $p$ twisted group algebra and let $N \triangleleft G$. Assume that $\mathbb{O}_{p}(G)$ is finite and that $G / N$ has a finite normal series with f.c. quotients. If $\mathcal{J} K^{t}[N]=\mathcal{J} K^{t}\left[\mathbb{S}^{p}(N)\right] \cdot K^{t}[N]$, then

$$
\mathcal{J} K^{t}[G]=\mathcal{J} K^{t}\left[\mathbb{S}^{p}(G)\right] \cdot K^{t}[G] .
$$

Proof. As usual, the assumption that $\mathbb{O}_{p}(G)$ is finite is inherited by all normal subgroups of $G$. Suppose first that $G / N$ is an f.c. group. Since $\mathcal{J} K^{t}\left[\mathbb{S}^{p}(G)\right] \cdot K^{t}[G] \subseteq$ $\mathcal{J} K^{t}[G]$ by Lemma 7.3 , we need only prove the reverse inclusion. To this end, let $\alpha \in \mathcal{J} K^{t}[G]$ and let $H / N$ be a finite normal subgroup of $G / N$ which contains $(\operatorname{supp} \alpha) N$. Then $H \triangleleft G,|H: N|<\infty$ and $\alpha \in \mathcal{J} K^{t}[G] \cap K^{t}[H] \subseteq \mathcal{J} K^{t}[H]$.

If $|H: N|=n$, then by Lemma 7.2 and the hypothesis, we have

$$
\left(\mathcal{J} K^{t}[H]\right)^{n} \subseteq \mathcal{J} K^{t}[N] \cdot K^{t}[H]=\mathcal{J} K^{t}\left[\mathbb{S}^{p}(N)\right] \cdot K^{t}[H] .
$$

Furthermore, since $\mathbb{S}^{p}(N) \triangleleft \mathbb{S}^{p}(H)$, it follows that

$$
\left(\mathcal{J} K^{t}[H]\right)^{n} \subseteq \mathcal{J} K^{t}\left[\mathbb{S}^{p}(N)\right] \cdot K^{t}[H] \subseteq \mathcal{J} K^{t}\left[\mathbb{S}^{p}(H)\right] \cdot K^{t}[H]
$$

by Lemma 7.3. But $\mathbb{O}_{p}(H)$ is finite, so $\mathcal{J} K^{t}\left[\mathbb{S}^{p}(H)\right] \cdot K^{t}[H]$ is a semiprime ideal by Proposition 8.3. Hence, we must have $\mathcal{J} K^{t}[H] \subseteq \mathcal{J} K^{t}\left[\mathbb{S}^{p}(H)\right] \cdot K^{t}[H]$. Again, by Lemma 7.3, this yields

$$
\alpha \in \mathcal{J} K^{t}[H] \subseteq \mathcal{J} K^{t}\left[\mathbb{S}^{p}(H)\right] \cdot K^{t}[H] \subseteq \mathcal{J} K^{t}\left[\mathbb{S}^{p}(G)\right] \cdot K^{t}[G],
$$

and the reverse inclusion is proved.

The general result now follows immediately from the above by induction on the length of the given normal series for $G / N$ with f.c. quotients.

\section{Crossed Products}

If $G$ is a locally finite group, then we know from Lemma 7.1 that $\mathcal{J} K^{t}[G]$ is a nil ideal. Of course, there is another way to see this fact. Indeed, since $K^{t}[G]$ is locally finite dimensional, it is clearly an algebraic algebra, and such algebras always have a nil Jacobson radical (see [P5, Lemma 2.3.12]). The point about studying algebraic algebras is that this property is inherited by subalgebras and by homomorphic images. Consequently, all such algebras $R$ involved in $K^{t}[G]$ necessarily satisfy $\mathcal{J} R=\mathcal{M} R$ where, for an arbitrary ring $R$, we let $\mathcal{M} R$ denote its nil radical, namely its unique largest nil ideal. (Recall that $\mathcal{N} R$ is the nilpotent radical of $R$, as defined in Section 1.) 
Now let $R$ be any ring and let $G$ be any group. Then a crossed product $R * G$ of $G$ over $R$ is an associative ring having a copy $\bar{G}$ of $G$ as a left $R$-basis. In other words, every element $\alpha$ of $R * G$ is uniquely a finite sum $\alpha=\sum_{x \in G} r_{x} \bar{x}$ with coefficients $r_{x} \in R$ and with support defined by $\operatorname{supp} \alpha=\left\{x \in G \mid r_{x} \neq 0\right\}$. Addition in $R * G$ is as expected, and multiplication is determined by the twisting and action rules below. Specifically, if $x, y \in G$, then

(twisting) $\quad \bar{x} \bar{y}=\tau(x, y) \overline{x y}$

where $\tau$ is a map from $G \times G$ to the group of units $U(R)$ of $R$. Furthermore, if $x \in G$ and $r \in R$, then

(action)

$$
r \bar{x}=\bar{x} r^{\sigma(g)}
$$

where $\sigma$ is a map from $G$ to $\operatorname{Aut}(R)$. Note that $\tau$ and $\sigma$ are not group homomorphisms in general. The relations they are assumed to satisfy are precisely equivalent to the associativity of the ring, and we may suppose that $\overline{1}=1$ is the identity element of $R * G$.

Obviously, any group algebra or twisted group algebra is a crossed product. Furthermore, suppose $K^{t}[G]$ is given with $N \triangleleft G$ and let $S$ be a transversal for $N$ in $G$. Observe that the elements of $S$ act on $R=K^{t}[N]$ by $r^{s}=\bar{s}^{-1} r \bar{s}$, and that if $s_{1}, s_{2} \in S$, then there exist $s_{3} \in S$ and $u \in N$ with $s_{1} s_{2}=u s_{3}$. Since $K^{t}[G]=\oplus \sum_{s \in S} K^{t}[N] \bar{s}$ and since there is a natural one-to-one correspondence between the elements of $S$ and those of $G / N$, it is now clear that $K^{t}[G]=R *(G / N)$ is a crossed product of $G / N$ over $R=K^{t}[N]$. In particular, crossed products can be used to study extension problems, namely the lifting of properties from $K^{t}[N]$ to $K^{t}[G]$. Note that, if $R * G$ is any crossed product and if $N \triangleleft G$, then the preceding argument also shows that $R * G=(R * N) *(G / N)$ where $R * N$ is the uniquely determined sub-crossed product of $R * G$ given by all those elements having support in $N$ (see [P7, Lemma 1.3]).

Again, let the crossed product $R * G$ be given. If $I \triangleleft R$ and $x \in G$, then $I^{\sigma(x)}=$ $\bar{x}^{-1} I \bar{x}$ is clearly an ideal of $R$ which we denote by $I^{x}$. Furthermore, if $x, y \in G$, then $\left(I^{x}\right)^{y}=I^{x y}$, since the conjugation action of $U(R)$ on the ideals of $R$ is trivial. In other words, there is a well-defined permutation action of $G$ on the two-sided ideals of $R$. Note that, if $I$ is $G$-stable, then we write $I * G$ for $I(R * G)$, the set of elements of $R * G$ with all coefficients in $I$, and it is easy to see that $I * G \triangleleft R * G$. Indeed, there is a natural crossed product $(R / I) * G$ which is obtained from $R * G$ via the epimorphism $\sum_{x} r_{x} \bar{x} \mapsto \sum_{x}\left(r_{x}+I\right) \bar{x}$ and this map has kernel $I * G$.

Two basic properties related to the Jacobson radical are as follows.

Lemma 9.1. Let $R * G$ be a crossed product.

i. $J=\mathcal{J} R$ is a $G$-stable ideal of $R$ and $J * G \subseteq \mathcal{J}(R * G)$ if $G$ is locally finite.

ii. If $H$ is a subgroup of $G$, then $\mathcal{J}(R * G) \cap R * H \subseteq \mathcal{J}(R * H)$.

Proof. (i) Since $\sigma: G \rightarrow \operatorname{Aut}(R)$ and since $J=\mathcal{J} R$ is a characteristic ideal of $R$, it follows that $J$ is $G$-stable and, by [P7, Theorem 4.2], $J * G \subseteq \mathcal{J}(R * G)$ at least when $G$ is finite. Finally, suppose $G$ is locally finite and let $\alpha$ be an arbitrary element of $J * G \triangleleft R * G$. Then there exists a finite subgroup $A$ of $G$ with $\alpha \in J * A \subseteq \mathcal{J}(R * A)$. In particular, $\alpha$ is quasi-regular, so $J * G$ is a quasi-regular ideal of $R * G$ and therefore it is contained in $\mathcal{J}(R * G)$.

(ii) Let $V$ be the set of elements of $R * G$ with support disjoint from $H$. Then $R * G=R * H \oplus V$, a decomposition as $(R * H, R * H)$-bimodules. It now follows 
easily that if $\alpha \in R * H$ is invertible in $R * G$, then its inverse is in $R * H$. Similarly, if $\alpha \in R * H$ is quasi-regular in $R * G$, then its quasi-inverse is in $R * H$. Finally, note that $J \cap R * H$ is an ideal of $R * H$ consisting of elements quasi-regular in $R * G$. Consequently, this is a quasi-regular ideal of $R * H$ and hence it is contained in $\mathcal{J}(R * H)$.

In this and the next section, we will be concerned with a particular extension problem. To be precise, let $K^{t}[G]$ be a twisted group algebra of the locally finite group $G$ over the field $K$ of characteristic $p$, and let $N \triangleleft G$ with $G / N$ an infinite simple, finitary linear group. Then the goal is to show that if $K^{t}[N]$ satisfies the conclusion of our main theorem, then so does $K^{t}[G]$. For example, we might wish to show that if $\mathcal{J} K^{t}[N]=0$, then $\mathcal{J} K^{t}[G]=0$. Of course, this special case has already been proved in [P7], but we mention this fact to point out that it is not a result about crossed products $R *(G / N)$ with $R$ semiprimitive. Indeed, we have the following elementary but quite informative example.

Lemma 9.2. Let $G$ be an arbitrary group containing an element of prime order $p$. Then there exist a semiprimitive commutative algebra $R$ over a field of characteristic $p$ and a crossed product $R * G$, such that $R * G$ is not semiprime. In particular, $R * G$ is not semiprimitive.

Proof. Let $H$ be the given subgroup of $G$ of order $p$ and let $K$ be a field of characteristic $p$. If $\Omega$ denotes the set of right cosets of $H$ in $G$, then $G$ permutes $\Omega$ by right multiplication, and we let $\omega_{0} \in \Omega$ correspond to the coset $H$. Thus $H=G_{\omega_{0}}=\left\{g \in G \mid \omega_{0} g=\omega_{0}\right\}$.

Now let $R$ be the (complete) direct product $\prod_{\omega \in \Omega} K_{\omega}$, where each $K_{\omega}$ is a copy of $K$. Then $R$ is a semiprimitive $K$-algebra, it is in fact von Neumann regular, and the permutation action of $G$ on $\Omega$ extends to an action of $G$ on $R$. In this way we obtain a homomorphism $\sigma: G \rightarrow \operatorname{Aut}(R)$ and we use $\sigma$ to form the skew group ring $R * G$. In other words, $R * G$ is a crossed product with parameter $\sigma$, as above, and with trivial twisting, so that $\tau(x, y)=1$ for all $x, y \in G$. By [P7, Lemma 1.2], such a construction always leads to an associative ring.

For each $\omega \in \Omega$, let $e_{\omega}$ denote the idempotent of $R$ which has a 1 for its $\omega$ coordinate and zeros elsewhere. Then $\bar{g}^{-1} e_{\omega} \bar{g}=e_{\omega g}$ and $e_{\omega} e_{\omega^{\prime}}=0$ if $\omega \neq \omega^{\prime}$. In particular, $e_{\omega_{0}}$ commutes with $\bar{H}$, and if $g \in G \backslash H$ and $r \in R$, then

$$
e_{\omega_{0}}(r \bar{g}) e_{\omega_{0}}=r \bar{g}\left(\bar{g}^{-1} e_{\omega_{0}} \bar{g}\right) e_{\omega_{0}}=r \bar{g} e_{\omega_{0} g} e_{\omega_{0}}=0 .
$$

It now follows easily that $e_{\omega_{0}}(R * G) e_{\omega_{0}}=e_{\omega_{0}} R * H \cong K[H]$ since $e_{\omega_{0}} R \cong K$ and the twisting is trivial. In particular, since $K$ has characteristic $p$ and $|H|=p$, we conclude that $e_{\omega_{0}}(R * G) e_{\omega_{0}}$ is not semiprime, and therefore neither is $R * G$.

Having finished this long introduction, we now begin our work on the extension problem. Again, all groups considered here are assumed to be locally finite. The following is the obvious crossed product generalization of the main result of [P13].

Proposition 9.3. Let $R * G$ be a crossed product of the locally finite group $G$ over the prime ring $R$ of characteristic $p>0$. If $\mathcal{M} R=0$ and $\mathbb{S}^{p}(G)=1$, then $\mathcal{M}(R * G)=0$.

Proof. Let $I=\mathcal{M}(R * G)$ and suppose, by way of contradiction, that $I \neq 0$. We use basic results of [FMo] and [Mo] to describe the structure of $R * G$ with $R$ a prime coefficient ring (see [P7, Proposition 12.4]). To start with, let $S$ denote the 
symmetric Martindale ring of quotients of $R$. Then $S$ is a prime ring containing $R$, and $R * G$ extends to a unique crossed product $S * G$. In particular, $G$ "acts" on $S$ and we let $G_{\text {inn }}$ denote the set of all $g \in G$ such that conjugation by $\bar{g}$ induces an inner automorphism on $S$. Then $G_{\text {inn }} \triangleleft G$ and $S * G_{\text {inn }}=S \otimes_{F} E$ where $F=\mathbb{Z}(S)$ is a field and where $E=\mathbb{C}_{S * G}(S)$ is isomorphic to some twisted group algebra $F^{t}\left[G_{\text {inn }}\right]$. Since $\mathbb{S}^{p}(G)=1$ and $G_{\text {inn }} \triangleleft G$, it follows that $\mathbb{S}^{p}\left(G_{\text {inn }}\right)=1$. Consequently, since $F$ is clearly a field of characteristic $p,\left[\mathrm{P} 13\right.$, Theorem 1.4] implies that $E \cong F^{t}\left[G_{\text {inn }}\right]$ is semiprimitive.

Now let

$$
I^{\prime}=\{\alpha \in E \mid A \otimes \alpha \subseteq I \text { for some } 0 \neq A \triangleleft R\} .
$$

Then it follows from [P7, Proposition 12.5] that $I^{\prime} \neq 0$, and it is a standard fact that $I^{\prime} \triangleleft E$. Indeed, if $\alpha, \beta \in I^{\prime}$, then there exist $0 \neq A, B \triangleleft R$ with $A \otimes \alpha$ and $B \otimes \beta$ contained in $I$. Since the primeness of $R$ implies that $A \cap B \neq 0$ and since $(A \cap B) \otimes(\alpha+\beta) \subseteq I$, we have $\alpha+\beta \in I^{\prime}$. Furthermore, if $\gamma \in E$, then by [P7, Proposition 10.4(iii)] there exists a nonzero ideal $C \triangleleft R$ with $C \otimes \gamma \subseteq R * G$ inn $\subseteq R * G$. Thus, since $I$ is an ideal of $R * G$, we have

$$
\begin{aligned}
& A C \otimes \alpha \gamma=(A \otimes \alpha)(C \otimes \gamma) \subseteq I, \\
& C A \otimes \gamma \alpha=(C \otimes \gamma)(A \otimes \alpha) \subseteq I,
\end{aligned}
$$

and the primeness of $R$ implies that $\alpha \gamma, \gamma \alpha \in I^{\prime}$.

Finally, since $I^{\prime}$ is a nonzero ideal of the semiprimitive $\operatorname{ring} E \cong F^{t}\left[G_{\text {inn }}\right]$, we can choose an element $\alpha \in I^{\prime}$ which is not nilpotent. By definition, $A \otimes \alpha \subseteq I$ for some $0 \neq A \triangleleft R$ and, since $\mathcal{M} R=0$, we can also choose an element $a \in A$ which is not nilpotent. But then the element $a \otimes \alpha \in I$ is clearly not nilpotent, and this contradicts the fact that $I=\mathcal{M}(R * G)$.

Again, we use the techniques of [FMo] and [Mo].

Lemma 9.4. Let $R$ be a simple ring whose center $K$ is an algebraically closed field of characteristic $p$, and let $G$ be a locally finite $p$-group. If $R * G$ is any crossed product of $G$ over $R$, then $R * G / \mathcal{J}(R * G)$ is a simple ring.

Proof. Let $G_{\text {inn }}$ denote the set of all $g \in G$ such that conjugation by $\bar{g}$ induces an inner automorphism on $R$. Then $G_{\text {inn }} \triangleleft G$ and $R * G_{\text {inn }}=R \otimes K^{t}\left[G_{\text {inn }}\right.$ ) (see [P7, Proposition 12.4]). Furthermore, since $K$ is an algebraically closed field of characteristic $p$ and since $G$ is a locally finite $p$-group, it follows that $K^{t}\left[G_{\text {inn }}\right]=$ $K\left[G_{\text {inn }}\right]$ (see for example [P13, Lemma 3.2(i)]). In particular, if $I$ denotes the augmentation ideal of $K\left[G_{\text {inn }}\right]$, then $I$ is nil and $K\left[G_{\text {inn }}\right] / I \cong K$.

Set $J=R \otimes I \triangleleft R \otimes K\left[G_{\mathrm{inn}}\right]=R * G_{\mathrm{inn}}$. Then certainly $R * G_{\mathrm{inn}} / J$ is naturally isomorphic to $R$, and $J$ is a nil ideal since $I$ is locally nilpotent. With all this, we conclude that $J=\mathcal{J} S$, where we set $S=R * G_{\text {inn. }}$ Finally, note that $R * G=\left(R * G_{\mathrm{inn}}\right) *\left(G / G_{\mathrm{inn}}\right)=S * H$, where $H=G / G_{\mathrm{inn}}$, and that $J$ is an $H$-stable ideal of $S$ with $J * H \subseteq \mathcal{J}(S * H)$ by Lemma 9.1(i). Furthermore, $(S * H) /(J * H) \cong(S / J) * H \cong R * H$, and $H=G / G_{\text {inn }}$ is totally outer in its action on $R$. Thus, $R * H$ is simple, by [P7, Corollary 12.6], and therefore it follows that $\mathcal{J}(R * G)=J * H$ and that $(R * G) / \mathcal{J}(R * G) \cong R * H$ is a simple ring.

When the coefficient $\operatorname{ring} R$ is not prime, there are other methods which can be used. For example, we have 
Lemma 9.5. Let $R * G$ be a crossed product, let $Q$ be a prime ideal of $R$ with stabilizer $H=\left\{g \in G \mid Q=Q^{g}\right\}$, and set $M=\bigcap_{g \in G} Q^{g}$. If the crossed product $(R / Q) * H=(R * H) /(Q * H)$ satisfies one of the properties

i. it has nil radical 0 ,

ii. it is semiprime, or

iii. it is prime,

then $(R * G) /(M * G) \cong(R / M) * G$ also satisfies that property. In addition, if the crossed product $(R / Q) * H$ is simple, then $(R * G) /(M * G)$ is semiprimitive.

Proof. It clearly suffices to assume that $M=0$ and hence that $\bigcap_{g \in G} Q^{g}=0$. We first show that every nonzero ideal $I$ of $R * G$ determines a nonzero ideal $I^{\prime}$ of $(R / Q) * H$. To this end, we note that $Q(R * G)$ is a right ideal of $R * G$ and that it is a left $R * H$-module since $Q$ is $H$-stable. Consequently, if we define $S=$ $R * H+Q(R * G) \subseteq R * G$, then $S$ is a subring of $R * G$ with $Q(R * G)$ a two-sided ideal. Furthermore, since $Q(R * G) \cap R * H=Q * H$, it follows that

$$
\frac{S}{Q(R * G)}=\frac{R * H+Q(R * G)}{Q(R * G)} \cong \frac{R * H}{Q * H}=(R / Q) * H .
$$

Now let $I$ be any nonzero ideal of $R * G$ and let $\alpha$ be a nonzero element of this ideal. If $x \in \operatorname{supp} \alpha$, then $\bar{x}^{-1} \alpha \in I$ and $1 \in \operatorname{supp} \bar{x}^{-1} \alpha$. Thus, by replacing $\alpha$ by $\bar{x}^{-1} \alpha$ if necessary, we can assume that $1 \in \operatorname{supp} \alpha$, and let $0 \neq r \in R$ be its identity coefficient. Since $\bigcap_{g \in G} Q^{g}=0$, it follows that $r \notin Q^{g}$ for some $g \in G$, and then $\bar{g} \alpha \bar{g}^{-1} \in I$ has identity coefficient equal to $\bar{g} r \bar{g}^{-1} \notin Q$. Thus, by replacing $\alpha$ by $\bar{g} \alpha \bar{g}^{-1}$ if necessary, we can further assume that $r \notin Q$.

Let $X$ be the finite set $X=\operatorname{supp} \alpha \backslash H$ and suppose, by way of contradiction, that $r \cdot \bigcap_{x \in X} Q^{x} \subseteq Q$. Then, since $r \notin Q$ and $Q$ is prime, it follows that $Q \supseteq Q^{y}$ for some $y \in X$, and it is a standard fact that this forces $Q$ to equal $Q^{y}$. Indeed, since $G$ is a periodic group, $y^{n}=1$ for some $n \geq 1$ and, by applying various powers of $y$ to the inclusion $Q \supseteq Q^{y}$, we obtain

$$
Q \supseteq Q^{y}, Q^{y} \supseteq Q^{y^{2}}, Q^{y^{2}} \supseteq Q^{y^{3}}, \ldots, Q^{y^{n-1}} \supseteq Q^{y^{n}}=Q .
$$

Thus $Q=Q^{y}$ and $y \in H$, a contradiction by the definition of $X$. In other words, we can now choose $t \in \bigcap_{x \in X} Q^{x}$ with $r t \notin Q$, and we consider the element $\alpha t \in I$.

Write $\alpha=\sum_{g} r_{g} \bar{g}$, and observe that

$$
\alpha t=\sum_{g} r_{g} \bar{g} t=\sum_{g} r_{g}\left(\bar{g} t \bar{g}^{-1}\right) \bar{g} .
$$

Furthermore, if $r_{g} \neq 0$ and $g \notin H$, then $g \in X$ and hence $t \in Q^{g}$. Thus $\bar{g} t \bar{g}^{-1} \in Q$, so $r_{g}\left(\bar{g} t \bar{g}^{-1}\right) \in Q$, and we conclude that $\alpha t \in R * H+Q(R * G)=S$. In addition, the identity coefficient of $\alpha t$ is $r t \notin Q$ and therefore $\alpha t \notin Q(R * G)$. Since $\alpha t \in I$, it now follows that $I \cap S$ is an ideal of $S$ not contained in $Q(R * G)$, and therefore

$$
I^{\prime}=\frac{(I \cap S)+Q(R * G)}{Q(R * G)} \cong \frac{I \cap S}{I \cap Q(R * G)}
$$

is a nonzero ideal of $S / Q(R * G)=(R / Q) * H$.

The remainder of the argument is now quite simple. To start with, if $I$ is a nonzero nil ideal of $R * G$, then $I \cap S$ is nil and hence so is $I^{\prime} \neq 0$. Next, if $I^{2}=0$, then $(I \cap S)^{2}=0$, so $\left(I^{\prime}\right)^{2}=0$. Finally, if $A$ and $B$ are nonzero ideals of $R * G$ with $A B=0$, then $(A \cap S)(B \cap S)=0$ and consequently $A^{\prime} B^{\prime}=0$. This yields the three required implications in their contrapositive forms. 
Finally, suppose $R * G$ is not semiprimitive and let $0 \neq J=\mathcal{J}(R * G)$. Since $J$ is not comaximal with any proper right ideal of $R * G$, we see that $1 \notin J+Q(R * G)$ and hence $1 \notin(J \cap S)+Q(R * G)$. Thus $0 \neq J^{\prime}$ is a proper ideal of $(R / Q) * H$, and therefore $(R / Q) * H$ cannot be simple.

It is unfortunately not true that if $(R / Q) * H$ is simple, then $R * G$ must be simple. A suitable counterexample can be constructed using the argument of Lemma 9.2. Indeed, let $G$ be an infinite group, let $K$ be any field and let $G$ act regularly as a permutation group on $\Omega=G$. If we define $R$ to be the (complete) direct product $R=\prod_{\omega \in \Omega} K_{\omega}$, then $G$ acts on $R$ and we can again form the skew group ring $R * G$. Choose $\omega_{0} \in \Omega$ and let $P$ be the set of elements of $R$ with $\omega_{0}$-coordinate equal to 0 . Then $P$ is a maximal ideal of $R$ and $\bigcap_{g \in G} P^{g}=0$, since $P^{g}$ is clearly the set of elements of $R$ with $\omega_{0} g$-coordinate equal to 0 . Furthermore, the stabilizer of $P$ in $G$ is the identity group $H=1$, so $(R / P) * H=R / P \cong K$ is simple. On the other hand, if $I$ is the (weak) direct product of the $K_{\omega} \mathrm{s}$, then $I$ is a proper $G$-stable ideal of $R$, and hence $I * G$ is a proper ideal of $R * G$.

Next, we extend Lemma 9.4 to the case where the coefficient ring is not necessarily simple. Note that here we assume, in addition, that $G$ is abelian, and even so, we get a slightly weaker conclusion.

Lemma 9.6. Let $P \triangleleft R$ and assume that $R / P$ is a simple ring whose center is an algebraically closed field of characteristic $p$. In addition, let $R * G$ be a crossed product of the abelian p-group $G$ over $R$, and set $I=\bigcap_{g \in G} P^{g}$. If $J /(I * G)$ is the Jacobson radical of $(R * G) /(I * G) \cong(R / I) * G$, then $J$ is a prime ideal of $R * G$ with $J \cap R=I$.

Proof. It clearly suffices to assume that $I=0$ and therefore that $\bigcap_{g \in G} P^{g}=$ 0 . If $H$ is the stabilizer of $P$ in $G$ and if $L /(P * H)$ is the Jacobson radical of $(R * H) /(P * H) \cong(R / P) * H$, then Lemma 9.4 implies that $L$ is a maximal ideal of $R * H$. Furthermore, since $P$ is a maximal ideal of $R$, it is clear that $L \cap R=P$.

By assumption, $G$ is abelian and hence $H \triangleleft G$. Thus $R * G=(R * H) *(G / H)=$ $S *(G / H)$ and, of course, $L$ is an ideal of $S=R * H$. Set $M=\bigcap_{x \in G / H} L^{x}$, so that $M$ is $G / H$-stable, and consider the ideal $M *(G / H) \triangleleft S *(G / H)=R * G$. To start with, observe that if $L=L^{x}$ with $x=H g \in G / H$, then $g$ stabilizes $L \cap R=P$ and hence $g \in H$. In other words, the stabilizer of $L$ in $G / H$ is the identity group $W=1$. Thus since $(S / L) * W=S / L=(R * H) / L$ is simple, it follows from Lemma 9.5 that $(S *(G / H)) /(M *(G / H))$ is a prime, semiprimitive ring. In particular, $J=\mathcal{J}(R * G) \subseteq M *(G / H)$.

Next, we consider the ideal $M$ itself. Let $\alpha \in M$ and choose a finite subgroup $A$ of $H$ with $\alpha \in R * A$. Since $G$ is abelian, each $P^{g}$ is $H$-stable and if $L^{g}=\bar{g}^{-1} L \bar{g}$, then $L^{g} /\left(P^{g} * H\right)$ is the Jacobson radical of $(R * H) /\left(P^{g} * H\right) \cong\left(R / P^{g}\right) * H$. Consequently, Lemma 9.1(ii) yields

$$
\left(L^{g} /\left(P^{g} * H\right)\right) \cap\left(\left(R / P^{g}\right) * A\right) \subseteq \mathcal{J}\left(\left(R / P^{g}\right) * A\right)
$$

and, by [P7, Theorem 4.2],

$$
\mathcal{J}\left(\left(R / P^{g}\right) * A\right)^{|A|} \subseteq \mathcal{J}\left(R / P^{g}\right) * A=0
$$

since $R / P^{g}$ is simple and hence semiprimitive. Now $\alpha \in R * A$ and $\alpha \in M=$ $\bigcap_{x \in G / H} L^{x}$, so it follows from the above that $\alpha^{|A|} \in \bigcap_{g \in G} P^{g_{*}} A=0$. In other words, $M$ is a nil ideal of $S$, and hence $M \subseteq \mathcal{J} S$. 
Finally, by Lemma 9.1(i), it follows that

$$
M *(G / H) \subseteq(\mathcal{J} S) *(G / H) \subseteq \mathcal{J}(S *(G / H))=\mathcal{J}(R * G)
$$

and, with this, we see that $\mathcal{J}(R * G)=M *(G / H)$. Consequently, $\mathcal{J}(R * G)$ is a prime ideal of $R * G$ and, since

$$
\begin{aligned}
\mathcal{J}(R * G) \cap R & =(M *(G / H)) \cap R=M \cap R \\
& =\bigcap_{x \in G / H} L^{x} \cap R=\bigcap_{x \in G / H} P^{x}=0,
\end{aligned}
$$

the lemma is proved.

The following rather technical lemma is the culmination of the work of this section. It is precisely what we need to describe the Jacobson radicals of the twisted group algebras of certain critical groups.

Lemma 9.7. Let $R * G$ be a crossed product of prime characteristic $p$ and let $L$ be a normal abelian p-subgroup of $G$. Set $S=R * L$ and, as usual, view $R * G$ as $(R * L) *(G / L)=S *(G / L)$. Furthermore, let $P \triangleleft R$ with $R / P$ a simple ring whose center is an algebraically closed field. Now set $I=\bigcap_{x \in L} P^{x}$, let $H$ denote the stabilizer of $P$ in $G$, and assume that every subgroup $T / L$ of $G / L$ which contains $H L / L$ satisfies $\mathbb{S}^{p}(T / L)=1$. If $J /(I * L)=\mathcal{J}(S /(I * L))$ and if $Q=\bigcap_{y \in G / L} J^{y}$, then $Q \cap R=\bigcap_{g \in G} P^{g}$ and $(S *(G / L)) /(Q *(G / L))$ has nil radical 0 .

Proof. The preceding lemma implies that $J$ is a prime ideal of $S$ and that $J \cap R=$ $I=\bigcap_{x \in L} P^{x}$. Hence, since $Q=\bigcap_{y \in G / L} J^{y}$, it is clear that $Q \cap R=\bigcap_{g \in G} P^{g}$.

Now let $T / L$ be the stabilizer of the prime ideal $J \triangleleft S$ in $G / L$. Since $H$ stabilizes $P$ and $L \triangleleft G$, it follows that $H$ stabilizes $I$. Indeed, since $J /(I * L)=\mathcal{J}(S /(I * L))$, we see that $H$ also stabilizes $J$. Thus $T / L \supseteq H L / L$ and, by hypothesis, we have $\mathbb{S}^{p}(T / L)=1$. Consequently, Proposition 9.3 implies that $(S *(T / L)) /(J *(T / L)) \cong$ $(S / J) *(T / L)$ has nil radical 0 , and therefore $(S *(G / L)) /(Q *(G / L))$ also has nil radical 0 , by Lemma $9.5(\mathrm{i})$.

Finally, we need a simple observation whose proof is by now quite familiar.

Lemma 9.8. Let $R$ be a finite dimensional algebra over the algebraically closed field $K$ of characteristic $p$ and let $G$ be a finite p-group. If $I$ is a non-nil ideal of the $K$-algebra crossed product $R * G$, then $I$ contains a nonzero idempotent of $R$.

Proof. Suppose first that $R$ is semiprimitive and that $G$ transitively permutes the centrally primitive idempotents $e_{1}, e_{2}, \ldots, e_{n}$ of $R$. Since these idempotents yield an orthogonal decomposition of $1 \in R * G$, it follows from [P5, Lemma 6.1.6] that $R * G \cong M_{n}(e(R * G) e)$ where we set $e=e_{1}$, and it remains to consider the structure of $e(R * G) e$. To this end, note that if $g \in G$ and $r \in R$, then $e(r \bar{g}) e=r e \bar{g} e=r \bar{g} e^{\bar{g}} e$, and hence this expression is 0 if $g$ is not in the stabilizer $H$ of $e$. Thus $e(R * G) e=e(R * H) e=(e R) * H$, and $e R \cong M_{m}(K)$ since $K$ is algebraically closed. In particular, since all $K$-automorphisms of $M_{m}(K)$ are inner, it follows that $(e R) * H \cong M_{m}(K) \otimes K^{t}[H] \cong M_{m}\left(K^{t}[H]\right)$ for some twisted group algebra $K^{t}[H]$. Now by hypothesis, $K$ has characteristic $p$ and $H$ is a $p$ group. Therefore $K^{t}[H] \cong K[H]$ is a local ring (see [P5, Lemma 1.2.10]), and consequently, $R * G \cong M_{m n}(K[H])$ is also local. In particular, if $I$ is a non-nil ideal of $R * G$, then $I=R * G$ and $1 \in I \cap R$. 
Next, we just assume that $R$ is a semiprimitive ring. Then $G$ permutes the centrally primitive idempotents of $R$ in orbits $\mathcal{O}_{1}, \mathcal{O}_{2}, \ldots, \mathcal{O}_{k}$, and we let $f_{i}$ denote the sum of the members of $\mathcal{O}_{i}$. Then $f_{i}$ is a central idempotent of $R * G$, and $R * G=\oplus \sum_{i=1}^{k} f_{i}(R * G)$. Furthermore, $f_{i}(R * G) \cong\left(f_{i} R\right) * G$ and note that $f_{i} R$ is semiprimitive and that $G$ transitively permutes its centrally primitive idempotents. Since $I=\oplus \sum_{i=1}^{k} f_{i} I$ and $I$ is not nil, it follows that $f_{j} I$ is not nil for some subscript $j$. Consequently, the result of the first paragraph implies that $f_{j} I$ contains the identity element of $\left(f_{j} R\right) * G$, namely $f_{j}$, and hence $f_{j} \in f_{j} I \cap f_{j} R \subseteq I \cap R$.

For the general case, let $J=\mathcal{J} R$ and note that $J * G$ is a nil ideal of $R * G$ by Lemma $9.1(\mathrm{i})$. Hence, $(I+J * G) /(J * G)$ is a non-nil ideal of $(R * G) /(J * G) \cong$ $(R / J) * G$, and, since $R / J$ is semiprimitive, it follows from the result of the previous paragraph that $I+J * G$ contains an element $r \in R$ such that $r+J$ is a nonzero idempotent of $R / J$. But idempotents can be lifted modulo $J$, so it follows that $I+J * G$ contains a nonzero idempotent $e \in R$. In other words, $I$ contains an element $\alpha=e+\beta$ with $\beta \in J * G$. Furthermore, since $e \alpha e \in I$ and $e \alpha e=e+e \beta e$, we can assume that $e$ and $\beta$ commute. Finally, since $K$ has characteristic $p$ and $\beta^{p^{t}}=0$ for some $t$, we conclude that $I$ contains $\alpha^{p^{t}}=e^{p^{t}}+\beta^{p^{t}}=e$, as required.

\section{Critical Triples}

The goal of this section is to describe the Jacobson radical $\mathcal{J} K^{t}[G]$ of the characteristic $p$ twisted group algebras $K^{t}[G]$ when $(G, C, H)$ is a critical triple, as defined in Section 6. As we saw in that section, there are two possibilities for the structure of $G$ according to whether the infinite irreducible $G$-modules which are involved in $C$ are primitive or not. The imprimitive case requires a certain amount of additional work, so we study it first. Indeed, we begin with the group $\tilde{G}$ as described in Proposition 6.6 and, for convenience, we repeat its rather unwieldy properties. Note that we have added the assumption that the index set $\mathcal{I}$ is countable.

For a fixed prime $p$, we say that the locally finite group $G$ is subcritical if it has a normal series $D \subseteq X \subseteq L \subseteq C \subseteq G$ such that

i. $G / C \cong \mathfrak{A}=$ FAlt $_{\mathcal{I}}$ where $\mathcal{I}$ is a countably infinite set.

ii. $L=C^{p}$, so that $C / L$ is a $p^{\prime}$-group.

iii. $D$ is a finite abelian $p^{\prime}$-group which is central in $G^{p}$.

iv. $L$ is an f.c. group, and $L / X$ is an abelian $p$-group.

v. There exist finite normal subgroups $X_{i}$ of $C$, for all $i \in \mathcal{I}$, with $\left(X_{i}\right)^{g}=X_{i g}$ where $i g$ is the image of $i \in \mathcal{I}$ under the permutation $C g \in \mathfrak{A}$.

vi. $D \subseteq X_{i} \subseteq X$ and $X / D$ is the (weak) direct product $\prod_{i \in \mathcal{I}}\left(X_{i} / D\right)$.

vii. $X_{j} / D \subseteq\left(\mathbb{C}_{L / D}\left(X_{i} / D\right)\right)^{p}$ for all distinct $i, j \in \mathcal{I}$.

As we will see, conditions like (vii) above and the fact that $L=C^{p}$ are needed because we are dealing with twisted group rings rather than with ordinary ones.

For the remainder of this section, $K$ will denote an algebraically closed field of characteristic $p>0$. The following result handles the subcritical groups with $D=1$ and is the key to the entire argument. Note that our proof here uses all of the work of the preceding section.

Lemma 10.1. Let $K$ be an algebraically closed field of characteristic $p>0$ and let $G$ be a subcritical group for the prime $p$ with structure as described above. If $K^{t}[G]$ is a twisted group algebra of $G$ over $K$ and if $D=1$, then $\mathcal{J} K^{t}[G]=\mathcal{J} K^{t}[L] \cdot K^{t}[G]$. 
Proof. Set $R=K^{t}[X]$ and $S=K^{t}[L]$, so that $S=R *(L / X)$ and $K^{t}[G]=$ $S *(G / L)=R *(G / X)$ in crossed product notation. Now let $\mathcal{Q}$ denote the set of all $G / L$-stable ideals $Q$ of $S$ such that

$$
\frac{K^{t}[G]}{Q *(G / L)}=\frac{S *(G / L)}{Q *(G / L)} \cong(S / Q) *(G / L)
$$

is a semiprimitive ring. Since $\mathcal{Q}$ is clearly closed under intersections, it has a unique minimal member $\tilde{Q}$ and, by Lemma $9.1(\mathrm{i})$,

$$
\tilde{Q} *(G / L) \supseteq \mathcal{J}(S *(G / L)) \supseteq(\mathcal{J} S) *(G / L) .
$$

In particular, $\tilde{Q} \supseteq \mathcal{J} S$, and consequently, if we show that $\tilde{Q} \subseteq \mathcal{J} S$, then the above displayed inclusions become equalities and we obtain

$$
\mathcal{J} K^{t}[G]=\mathcal{J}(S *(G / L))=(\mathcal{J} S) *(G / L)=\mathcal{J} K^{t}[L] \cdot K^{t}[G],
$$

as required. In other words, our goal here is to show that if $\alpha \in S \backslash \mathcal{J} S$, then there exists $Q \in \mathcal{Q}$ with $\alpha \notin Q$.

For this, we first need to consider the structure of $R=K^{t}[X]$. Note that $D=1$, so $X=\prod_{i \in \mathcal{I}} X_{i}$ is the (weak) direct product of the finite groups $X_{i}$. In particular, if $i \neq j$, then $X_{j}$ centralizes $X_{i}$. In fact, by assumption (vii), $X_{j} \subseteq$ $\left(\mathbb{C}_{L}\left(X_{i}\right)\right)^{p}$ and therefore, since $K$ is a field of characteristic $p$, Lemma 7.5 implies that $\bar{X}_{j}$ centralizes $K^{t}\left[X_{i}\right]$. It follows that if $\mathcal{K}$ is any finite subset of $\mathcal{I}$ and if $Y_{\mathcal{K}}=\prod_{k \in \mathcal{K}} X_{k} \subseteq X$, then $K^{t}\left[Y_{\mathcal{K}}\right]$ is equal to the tensor product over $K$ of its subalgebras $K^{t}\left[X_{k}\right]$ with $k \in \mathcal{K}$. In other words, $R$ is the "infinite tensor product" of its subalgebras $K^{t}\left[X_{i}\right]$ with $i \in \mathcal{I}$. Furthermore, suppose $P_{i}$ is a maximal ideal of $K^{t}\left[X_{i}\right]$. Then, since $K$ is algebraically closed, $P_{\mathcal{K}}=\sum_{k \in \mathcal{K}} P_{k} \cdot K^{t}\left[Y_{\mathcal{K}}\right]$ is a maximal ideal of $K^{t}\left[Y_{\mathcal{K}}\right]$, each maximal ideal of $K^{t}\left[Y_{\mathcal{K}}\right]$ is of this form, and $K^{t}\left[Y_{\mathcal{K}}\right] / P_{\mathcal{K}}$ has center $K$. Consequently, if we let $P=\sum_{i \in \mathcal{I}} P_{i} \cdot K^{t}[X]=\sum_{i \in \mathcal{I}} P_{i} R$, then $P \cap K^{t}\left[Y_{\mathcal{K}}\right]=P_{\mathcal{K}}$ and therefore $P$ is a maximal ideal of $R$. Indeed, $R / P$ is the sum of its simple subrings $\left(K^{t}\left[Y_{\mathcal{K}}\right]+P\right) / P \cong K^{t}\left[Y_{\mathcal{K}}\right] / P_{\mathcal{K}}$ over all such finite subsets $\mathcal{K}$ of $\mathcal{I}$, and this also implies that $K$ is the center of $R / P$.

Since $G / C \cong$ FAlt $_{\mathcal{I}}$ permutes the groups $X_{i}$ transitively, all such $X_{i}$ are isomorphic. Thus, we can define the integer $w$ by $w=\left|\operatorname{Aut}\left(X_{i}\right)\right| \cdot\left|\operatorname{Hom}\left(X_{i}, K^{\bullet}\right)\right|$ for any $i \in \mathcal{I}$, and it follows from Lemma 7.5 that if $g \in G$ normalizes $X_{i}$, then $\bar{g}^{w}$ centralizes $K^{t}\left[X_{i}\right]$.

Now let $\alpha \in S \backslash \mathcal{J} S$ be as above. Then $\alpha S$ is not a nil right ideal of $S$, so there exists $\beta \in S$ with $\alpha \beta$ not nilpotent. In particular, if $W$ is a finite subgroup of $L$ containing $\operatorname{supp} \alpha$ and $\operatorname{supp} \beta$, then $K^{t}[W] \alpha K^{t}[W]$ is a non-nil ideal of $K^{t}[W]$. Set $W_{0}=W \cap X$, and note that $K^{t}[W]=K^{t}\left[W_{0}\right] *\left(W / W_{0}\right)$ and that

$$
W / W_{0}=W /(W \cap X) \cong W X / X \subseteq L / X
$$

Thus $W / W_{0}$ is a finite $p$-group, and therefore Lemma 9.8 implies that the ideal $K^{t}[W] \alpha K^{t}[W]$ contains a nonzero idempotent $e \in K^{t}\left[W_{0}\right]$. Obviously, it now suffices to find an ideal $Q \in \mathcal{Q}$ with $e \notin Q$. Of course, $e \in K^{t}[X]$, so there exist finitely many $X_{i}$ s, say $X_{1}, X_{2}, \ldots, X_{s}$ such that, if $Y=X_{1} \times X_{2} \times \cdots \times X_{s}$, then $e \in K^{t}[Y]$. Furthermore, since $e$ is a nonzero idempotent of $K^{t}[Y]$, there exists a maximal ideal $P^{\prime}$ of this algebra not containing $e$. By our previous observations, this maximal ideal has the form $P^{\prime}=\sum_{i=1}^{s} P_{i} \cdot K^{t}[Y]$, where $P_{i}$ is a maximal ideal of $K^{t}\left[X_{i}\right]$ for $i=1,2, \ldots, s$. 
At this point, we use the fact that $\mathcal{I}$ is countably infinite to construct a family of maximal ideals $P_{i}$ of $K^{t}\left[X_{i}\right]$ for all $i \in \mathcal{I}$ extending the original set and with certain additional properties. To start with, relabel the elements of $\mathcal{I}$ as ordered pairs $\{a, b\}$, where $a=1,2, \ldots, s, b=0,1,2, \ldots$ and where $i=\{i, 0\}$ for $i=1,2, \ldots, s$. In other words, $Y=X_{\{1,0\}} \times X_{\{2,0\}} \times \cdots \times X_{\{s, 0\}}, P_{\{i, 0\}}$ is a maximal ideal of $K^{t}\left[X_{\{i, 0\}}\right]$, and $X=\prod_{\{i, j\}} X_{\{i, j\}}=\prod_{i=1}^{s} \prod_{j=0}^{\infty} X_{\{i, j\}}$. Now choose any (odd) prime $q$ which is strictly larger than the integer $w=\left|\operatorname{Aut}\left(X_{i}\right)\right| \cdot\left|\operatorname{Hom}\left(X_{i}, K^{\bullet}\right)\right|$ and, since $w$ and $q$ are relatively prime, let $m$ be an integer divisible by $w$ and congruent to 1 modulo $q$.

Since $G / C \cong \mathfrak{A}=\mathrm{FAlt}_{\mathcal{I}}$, there exists an element $g_{i, n} \in G$, for any $i=1,2, \ldots, s$ and $n=0,1,2, \ldots$ which corresponds to the $q$-cycle

$$
(\{i, n(q-1)\},\{i, n(q-1)+1\},\{i, n(q-1)+2\}, \ldots,\{i,(n+1)(q-1)\}) .
$$

Here, of course, we are using the ordered pair notation for the elements of $\mathcal{I}$. Note that the element $g_{i, n}$ normalizes all those $X_{\{a, b\}}$ for which $\{a, b\}$ does not appear in its $q$-cycle, and therefore, since $w \mid m$, we see that $\bar{g}_{i, n}^{m}$ centralizes each such twisted group algebra $K^{t}\left[X_{\{a, b\}}\right]$. Furthermore, since $m$ is congruent to 1 modulo $q$, we see that $g_{i, n}^{m}$ and $g_{i, n}$ both give rise to the same $q$-cycle in $\mathfrak{A}$. Thus, by replacing $g_{i, n}$ by $g_{i, n}^{m}$ if necessary, we can now assume that $\bar{g}_{i, n}$ centralizes all those $K^{t}\left[X_{\{a, b\}}\right]$ with $\{a, b\}$ not appearing in its cycle, and we can assume that $\bar{g}_{i, n}^{q}$ centralizes $R$.

Note that the permutation $g_{i, n}^{q-1} C \in \mathfrak{A}$ maps $\{i, n(q-1)\}$ to $\{i,(n+1)(q-1)\}$ and therefore $g_{i, 0}^{q-1} g_{i, 1}^{q-1} \cdots g_{i, n-1}^{q-1} C$ sends $\{i, 0\}$ to $\{i, n(q-1)\}$. Consequently, if $j \geq 0$ and if we write $j=n(q-1)+r$ uniquely, where the remainder $r$ satisfies $0 \leq r \leq q-2$, then

$$
g_{i, 0}^{q-1} g_{i, 1}^{q-1} \cdots g_{i, n-1}^{q-1} g_{i, n}^{r} C
$$

maps $\{i, 0\}$ to $\{i, j\}$, and we define $P_{\{i, j\}}$ to be the image of $P_{\{i, 0\}}$ under the conjugation action of the element

$$
\bar{g}_{i, 0}^{q-1} \bar{g}_{i, 1}^{q-1} \cdots \bar{g}_{i, n-1}^{q-1} \bar{g}_{i, n}^{r} \in K^{t}[G] .
$$

Certainly, $P_{\{i, j\}}$ is a maximal ideal of $K^{t}\left[X_{\{i, j\}}\right]$, and $P_{\{i, 0\}}=P_{i}$ for $i=1,2, \ldots, s$. In particular, if we set

$$
P=\sum_{\{i, j\}} P_{\{i, j\}} \cdot K^{t}[X]=\sum_{\{i, j\}} P_{\{i, j\}} R,
$$

then our previous observations imply that $P$ is a maximal ideal of $R$ and that $R / P$ has center $K$. Furthermore,

$$
P \cap K^{t}[Y]=\sum_{i=1}^{s} P_{\{i, 0\}} \cdot K^{t}[Y]=\sum_{i=1}^{s} P_{i} \cdot K^{t}[Y]=P^{\prime}
$$

and therefore $e \notin P$.

Let $H / X=\left\{g X \in G / X \mid P^{g}=P\right\}$ be the stabilizer of $P$ in $G / X$. Then the construction of $P$ and the nature of the action of $\bar{g}_{i, n}$ on $K^{t}[X]=R$ imply that $g_{i, n} X \in H / X$ for all $i, n$. Indeed, conjugation by $\bar{g}_{i, n}$ cycles the maximal ideals $P_{\{i, n(q-1)\}}, P_{\{i, n(q-1)+1\}}, \ldots, P_{\{i,(n+1)(q-1)\}}$ and centralizes all the rest. Now fix $a=1,2, \ldots, s$, and set $\mathcal{I}_{a}=\{\{a, b\} \mid b=0,1,2, \ldots\}$. Since the permutations $g_{a, b} C$ 
for $b=0,1,2, \ldots$ are $q$-cycles which generate a transitive subgroup of FAlt $_{\mathcal{I}_{a}}$, it follows from Lemma 6.7 that these elements generate FAlt $_{\mathcal{I}_{a}}$, and therefore

$$
\text { FAlt }_{\mathcal{I}} \supseteq H C / C \supseteq \text { FAlt }_{\mathcal{I}_{1}} \times \text { FAlt }_{\mathcal{I}_{2}} \times \cdots \times \text { FAlt }_{\mathcal{I}_{s}} .
$$

In particular, if $T$ is any subgroup of $G$ containing $H$, then Lemma 6.8 implies that $\mathbb{S}(T /(T \cap C)) \cong \mathbb{S}(T C / C)=1$. Furthermore, since

$$
\frac{T}{T \cap C} \cong \frac{T /(T \cap L)}{(T \cap C) /(T \cap L)}
$$

it follows that

$$
\mathbb{S}^{p}(T /(T \cap L)) \subseteq(T \cap C) /(T \cap L) \cong(T \cap C) L / L \subseteq C / L
$$

and hence, since $C / L$ is a $p^{\prime}$-group, we have $\mathbb{S}^{p}(T L / L) \cong \mathbb{S}^{p}(T /(T \cap L))=1$.

We can now apply Lemma 9.7 to the crossed product $K^{t}[G]=R *(G / X)=$ $S *(G / L)$ and maximal ideal $P$ of $R$. Since the stabilizer of $P$ in $G / X$ has the properties described above, it follows that there is a $G / L$-stable ideal $Q$ of $S$ such that $Q \cap R=\bigcap_{g X \in G / X} P^{g}$ and such that $(S *(G / L)) /(Q *(G / L))$ has nil radical 0 . But $K^{t}[G]$ is algebraic over $K$, so the Jacobson radicals of all algebras involved in it are necessarily nil. Consequently, $(S *(G / L)) /(Q *(G / L))$ is a semiprimitive ring and therefore, by definition, $Q \in \mathcal{Q}$. Finally, since $e \notin P$ and since $Q \cap R \subseteq P$, we conclude that $e \notin Q$. As we indicated earlier, this yields the result.

It is now a simple matter to deal with the remaining subcritical groups. Indeed, we have

Lemma 10.2. Let $K$ be an algebraically closed field of characteristic $p>0$ and let $G$ be a subcritical group for the prime $p$ with structure as described above. If $K^{t}[G]$ is a twisted group algebra of $G$ over $K$, then

$$
\mathcal{J} K^{t}[G]=\mathcal{J} K^{t}[L] \cdot K^{t}[G]=\mathcal{J} K^{t}\left[\mathbb{S}^{p}(G)\right] \cdot K^{t}[G] .
$$

Proof. Let $E=\mathbb{C}_{G}^{t}(D)$ so that $E \triangleleft G$ and $|E / G|<\infty$. Indeed, since $G / \mathbb{C}_{G}(D)$ is a $p^{\prime}$-group by assumption (iii), and since $\mathbb{C}_{G}(D) / \mathbb{C}_{G}^{t}(D)$ is a $p^{\prime}$-group by Lemma 7.5 , we see that $G / E$ is a finite $p^{\prime}$-group and thus $E \supseteq G^{p} \supseteq C^{p}=L$. Furthermore, since $\mathfrak{A}=$ FAlt $_{\mathcal{I}}$ has no proper subgroup of finite index, it follows that $E C=G$ and hence that $E /(E \cap C) \cong G / C \cong \mathfrak{A}$. With this, it is now clear that $E$ is a subcritical group with corresponding normal series $D \subseteq X \subseteq L \subseteq C \cap E \subseteq E$, and hence the group $E / D$ is also subcritical.

Now $D \subseteq E=\mathbb{C}_{G}^{t}(D)$, so $K^{t}[D]$ is commutative and, in fact, central in $K^{t}[E]$. Thus, since $D$ is a finite $p^{\prime}$-group and $K$ is an algebraically closed field of characteristic $p$, it follows that $K^{t}[D]=\oplus \sum_{i=1}^{n} e_{i} K$, where the $e_{i}$ s are the centrally primitive idempotents of $K^{t}[D]$. Indeed, since the $e_{i}$ s are central in $K^{t}[E]$, they give rise to the ring direct sum $K^{t}[E]=\oplus \sum_{i=1}^{n} e_{i} K^{t}[E]$ and, since $e_{i} K^{t}[D]=e_{i} K$, it is clear that there exist natural isomorphisms $e_{i} K^{t}[E] \cong K^{t_{i}}[E / D]$, where the latter are appropriate twisted group algebras of $E / D$.

By the preceding lemma, $\mathcal{J} K^{t_{i}}[E / D]=\mathcal{J} K^{t_{i}}[L / D] \cdot K^{t_{i}}[E / D]$ and therefore, via the natural isomorphism, we have $\mathcal{J}\left(e_{i} K^{t}[E]\right)=\mathcal{J}\left(e_{i} K^{t}[L]\right) \cdot K^{t}[E]$. In particular, since $\mathcal{J} K^{t}[E]=\oplus \sum_{i=1}^{n} \mathcal{J}\left(e_{i} K^{t}[E]\right)$ and $\mathcal{J} K^{t}[L]=\oplus \sum_{i=1}^{n} \mathcal{J}\left(e_{i} K^{t}[L]\right)$, it follows that

$$
\mathcal{J} K^{t}[E]=\oplus \sum_{i=1}^{n} \mathcal{J}\left(e_{i} K^{t}[L]\right) \cdot K^{t}[E]=\mathcal{J} K^{t}[L] \cdot K^{t}[E]
$$


In addition, since $G / E$ is a finite $p^{\prime}$-group, Lemma 7.2 yields

$$
\mathcal{J} K^{t}[G]=\mathcal{J} K^{t}[E] \cdot K^{t}[G]=\mathcal{J} K^{t}[L] \cdot K^{t}[G],
$$

the required description for $\mathcal{J} K^{t}[G]$. Finally, note that $L=C^{p}$ is a normal f.c. subgroup of $G$ generated by $p$-elements, so certainly $L \subseteq \mathbb{S}^{p}(G)$. On the other hand, $C / L$ is a $p^{\prime}$-group and $\mathbb{S}(G / C) \cong \mathbb{S}(\mathfrak{A})=1$ by Lemma 6.7. Thus $L=\mathbb{S}^{p}(G)$ and the result follows.

Finally, we compute $\mathcal{J} K^{t}[G]$ when $(G, C, H)$ is a critical triple.

Proposition 10.3. Let $K$ be an algebraically closed field of characteristic $p>0$ and let $(G, C, H)$ be a critical triple for the prime $p$. If $G / C$ is countably infinite and if $K^{t}[G]$ is any twisted group algebra, then $\mathcal{J} K^{t}[G]=\mathcal{J} K^{t}\left[\mathbb{S}^{p}(G)\right] \cdot K^{t}[G]$.

Proof. We use the structure of $G$ as described in Lemmas 6.2 and 6.3. Suppose first that all infinite irreducible $\bar{C}_{r}$ are primitive $G$-modules. Then Lemma 6.3 implies that $G$ has normal subgroups $\tilde{C} \subseteq \tilde{G}$ with $\left|G^{(6)}: \tilde{G}\right|<\infty, \tilde{G} / \tilde{C}$ infinite simple, and with $\tilde{C}$ a nilpotent $p^{\prime}$-group. In this case, Lemma 7.3 and the main result of [P10] imply that $\mathcal{J} K^{t}\left[\mathbb{S}^{p}(\tilde{G})\right] \cdot K^{t}[\tilde{G}] \subseteq \mathcal{J} K^{t}[\tilde{G}]=0$, and therefore the latter two ideals are equal. Thus, since $G / \tilde{G}$ has a finite normal series with f.c. quotients, it follows from Proposition 8.5 that $\mathcal{J} K^{t}[G]=\mathcal{J} K^{t}\left[\mathbb{S}^{p}(G)\right] \cdot K^{t}[G]$, as required.

On the other hand, suppose that some $\bar{C}_{r}$ is an infinite irreducible and imprimitive $G$-module. Then, since $G / C$ is countable, Proposition 6.6 implies that $G$ has a normal subgroup $\tilde{G}$ with $\left|G^{(6)}: \tilde{G}\right|<\infty$ and with $\tilde{G}$ subcritical for the prime $p$. In this case, the preceding lemma implies that $\mathcal{J} K^{t}[\tilde{G}]=\mathcal{J} K^{t}\left[\mathbb{S}^{p}(\tilde{G})\right] \cdot K^{t}[\tilde{G}]$, and a second application of Proposition 8.5 yields the result.

\section{Simple Extensions}

The first part of this section is devoted to the study of simple group extensions. Specifically, let $K^{t}[G]$ be given and let $N \triangleleft G$ with $G / N$ a simple finitary linear group. If $K^{t}[N]$ satisfies the conclusion of our main theorem, then we show in Proposition 11.3 that $K^{t}[G]$ also satisfies that conclusion. We then go on, in Lemmas 11.4 and 11.5, to obtain a close approximation to Theorem 1.2.

To start with, let $K^{t}[G]$ be an arbitrary twisted group algebra. Recall that if $\alpha=\sum a_{x} \bar{x} \in K^{t}[G]$, then the support of $\alpha$ is the finite subset of $G$ given by $\operatorname{supp} \alpha=\left\{x \in G \mid a_{x} \neq 0\right\}$. In addition, we call $H=\langle\operatorname{supp} \alpha\rangle$ the supporting subgroup of $\alpha$. Clearly $H$ is the smallest subgroup of $G$ with $\alpha \in K^{t}[H]$ and, since $G$ is locally finite, $H$ is finite. We say that $\beta \in K^{t}[G]$ is a truncation of $\alpha$ if $\beta=\sum^{\prime} a_{x} \bar{x}$, where $\sum^{\prime}$ indicates a partial sum of the terms of $\alpha$. Thus $\operatorname{supp} \beta \subseteq \operatorname{supp} \alpha$, and the coefficients of $\alpha$ and of $\beta$ agree on the smaller set. Of course, $\beta$ is a proper truncation if $\beta \neq 0$ or $\alpha$.

Recall that, if $D$ is any subgroup of $G$, then there is a natural $K^{t}[D]$-bimodule projection map $\pi_{D}: K^{t}[G] \rightarrow K^{t}[D]$ given by

$$
\pi_{D}: \sum_{x \in G} a_{x} \bar{x} \mapsto \sum_{x \in D} a_{x} \bar{x} .
$$

Thus $\pi_{D}$ is the linear extension of the map $\bar{G} \rightarrow \bar{D} \cup\{0\}$ which is the identity on $\bar{D}$ and zero on $\bar{G} \backslash \bar{D}$. Clearly, $\pi_{D}(\alpha)$ is a truncation of $\alpha$. 
Now let $I \triangleleft K^{t}[G]$. We say that $0 \neq \alpha$ is a minimal element of $I$, if $\alpha \in I$ but no proper truncation of $\alpha$ is contained in $I$. These minimal elements generate $I$ in the following sense.

Lemma 11.1. If $I$ is an ideal of $K^{t}[G]$, then $I$ is the linear span of its minimal elements. Furthermore, I is the right (or left) ideal generated by those minimal elements having 1 in their support.

Proof. Let $I^{\prime} \subseteq I$ be the linear span of the minimal elements of $I$. If $\alpha \in I$, we show that $\alpha \in I^{\prime}$ by induction on $|\operatorname{supp} \alpha|$, the result being clear if $\alpha=0$ or if $\alpha$ is a minimal element of $I$. On the other hand, if $\alpha$ is not minimal, then there is a proper truncation $\beta$ of $\alpha$ contained in $I$. But then $\beta$ and $\alpha-\beta$ are elements of $I$ of smaller support size, so both of these are contained in $I^{\prime}$ and hence so is $\alpha=\beta+(\alpha-\beta)$. Finally, note that if $\gamma$ is a minimal element of $I$ and if $x \in \operatorname{supp} \gamma$, then $\gamma \bar{x}^{-1}$ is a minimal element having 1 in its support. Since $\gamma=\left(\gamma \bar{x}^{-1}\right) \bar{x} \in\left(\gamma \bar{x}^{-1}\right) K^{t}[G]$, the result follows.

In particular, if $I \neq 0$, then $I$ has minimal elements with 1 in their support. Next, we need a variant of a key idea used in [P12].

Lemma 11.2. Suppose $\mathcal{J} K^{t}[G] \neq 0$ and let $\alpha$ be a minimal element of this ideal having 1 in its support. Then there exists a finite subgroup $H$ of $G$ containing the supporting subgroup $\langle\operatorname{supp} \alpha\rangle$ such that

i. $H=\langle\operatorname{supp} \alpha\rangle^{H}$ and $H=H^{p}$,

ii. $\alpha$ is a minimal element of $\mathcal{J} K^{t}[H]$,

iii. if $N$ is any subgroup of $G$ normalized by $H$ and if $\mathcal{J} K^{t}[N]$ is nilpotent, then $H \subseteq \mathbb{D}_{G}(N)$,

iv. if $N$ is any subgroup of $G$ normalized by $H$ which satisfies both $\mathbb{O}_{p}(N)=1$ and $\mathcal{J} K^{t}[N]=\mathcal{J} K^{t}\left[\mathbb{S}^{p}(N)\right] \cdot K^{t}[N]$, then $H \subseteq \mathbb{S}^{p}(N H)$ and $H \subseteq \mathbb{D}_{G}\left(F^{*}\right)$, where $F^{*}=\mathbb{F}^{*}(N)$.

Proof. Let $\beta_{1}, \beta_{2}, \ldots, \beta_{k}$ be the finitely many proper truncations of $\alpha$. By definition, no $\beta_{i}$ is contained in $\mathcal{J} K^{t}[G]$, and hence the right ideals $\beta_{i} K^{t}[G]$ are not nil. In other words, we can choose elements $\gamma_{i} \in K^{t}[G]$ with $\beta_{i} \gamma_{i}$ not nilpotent. Now $G$ is locally finite, so there exists a finite subgroup $L \subseteq G$ which contains $\langle\operatorname{supp} \alpha\rangle$ and the supports of all $\gamma_{i}$. In particular, since $\mathcal{J} K^{t}[L]$ is nilpotent, it follows that $\beta_{i} \notin \mathcal{J} K^{t}[L]$ for all $i$.

Now let $H=\langle\operatorname{supp} \alpha\rangle^{[L]}$ be the subnormal closure of $\langle\operatorname{supp} \alpha\rangle$ in $L$. Then we know, at least, that $H=\langle\operatorname{supp} \alpha\rangle^{H}$ and that $\alpha \in \mathcal{J} K^{t}[G] \cap K^{t}[H] \subseteq \mathcal{J} K^{t}[H]$. Furthermore, since $H \triangleleft \triangleleft L$, it follows easily from Lemma 7.2 and induction that $\mathcal{J} K^{t}[H] \subseteq \mathcal{J} K^{t}[L]$. Thus, since $\beta_{i} \notin \mathcal{J} K^{t}[L]$, we have $\beta_{i} \notin \mathcal{J} K^{t}[H]$ and (ii) is proved. Note also that $\mathcal{J} K^{t}[H]=\mathcal{J} K^{t}\left[H^{p}\right] \cdot K^{t}[H]$, so $\pi_{H^{p}}(\alpha) \in \mathcal{J} K^{t}\left[H^{p}\right] \subseteq$ $\mathcal{J} K^{t}[H]$. In particular, since $1 \in \operatorname{supp} \alpha$, we see that $\pi_{H^{p}}(\alpha)$ is a nonzero truncation of $\alpha$ contained in $\mathcal{J} K^{t}[H]$, and therefore we conclude that $\pi_{H^{p}}(\alpha)=\alpha$. In other words, $\langle\operatorname{supp} \alpha\rangle \subseteq H^{p} \triangleleft H$ and, since $H=\langle\operatorname{supp} \alpha\rangle^{H}$, it follows that $H=H^{p}$.

For part (iii), suppose that $N$ is any subgroup of $G$ normalized by $H$ with $\mathcal{J} K^{t}[N]$ nilpotent. If $X=N H$, then $N$ is a normal subgroup of $X$ of finite index, so Lemma 7.2 implies that the radical $\mathcal{J} K^{t}[X]$ is also nilpotent. In particular, by Proposition 1.1, we have $\mathcal{J} K^{t}[X]=\mathcal{J} K^{t}[D] \cdot K^{t}[X]$ where $D=\Delta^{p}(X)$. Now $\alpha \in \mathcal{J} K^{t}[G] \cap K^{t}[X] \subseteq \mathcal{J} K^{t}[X]$ and therefore $\pi_{D}(\alpha)$ is a nonzero truncation of $\alpha$ contained in $\mathcal{J} K^{t}[D] \subseteq \mathcal{J} K^{t}[X]$. Thus $\pi_{D}(\alpha) \in \mathcal{J} K^{t}[X] \cap K^{t}[H] \subseteq \mathcal{J} K^{t}[H]$, so 
the minimal nature of $\alpha$ implies that $\pi_{D}(\alpha)=\alpha$. In other words, $\langle\operatorname{supp} \alpha\rangle \subseteq D \triangleleft X$ and therefore $H=\langle\operatorname{supp} \alpha\rangle^{H} \subseteq D$. But $N \subseteq X$, so the definition of $D$ implies that $\left|N: \mathbb{C}_{N}(h)\right|<\infty$ for all $h \in H$, and consequently $H \subseteq \mathbb{D}_{G}(N)$, as required.

Finally, suppose $H$ normalizes the group $N$ satisfying both $\mathbb{O}_{p}(N)=1$ and $\mathcal{J} K^{t}[N]=\mathcal{J} K^{t}\left[\mathbb{S}^{p}(N)\right] \cdot K^{t}[N]$. Again, let $X=N H$ and note that $N \triangleleft X$ with $|X / N|<\infty$. Thus Proposition 8.5 implies that $\mathcal{J} K^{t}[X]=\mathcal{J} K^{t}[S] \cdot K^{t}[X]$ where $S=\mathbb{S}^{p}(X)$. Now observe that $\alpha \in \mathcal{J} K^{t}[G] \cap K^{t}[X] \subseteq \mathcal{J} K^{t}[X]$ and therefore we see that $\pi_{S}(\alpha)$ is a nonzero truncation of $\alpha$ contained in $\mathcal{J} K^{t}[S] \subseteq \mathcal{J} K^{t}[X]$. Thus $\pi_{S}(\alpha) \in \mathcal{J} K^{t}[X] \cap K^{t}[H] \subseteq \mathcal{J} K^{t}[H]$, and the minimal nature of $\alpha$ again implies that $\pi_{S}(\alpha)=\alpha$. In other words, $\langle\operatorname{supp} \alpha\rangle \subseteq S \triangleleft X$, and consequently $H=\langle\operatorname{supp} \alpha\rangle^{H} \subseteq S=\mathbb{S}^{p}(N H)$. Furthermore, since $N \triangleleft N H$, we have $F^{*}=$ $\mathbb{F}^{*}(N) \subseteq \mathbb{F}^{*}(N H)$ by Lemma 4.3(iii). Thus, since $\mathbb{O}_{p}(N H)$ is finite, Lemma 4.4 applied to the group $\mathbb{S}^{p}(N H)$ shows that $H$ acts in a finitary manner on $\mathbb{F}^{*}(N H)$ and hence also on $F^{*}$.

We now solve the extension problem.

Proposition 11.3. Let $K^{t}[G]$ be given with $K$ algebraically closed and let $N \triangleleft G$ with $\mathbb{O}_{p}(N)=1$. Assume that $G / N$ is an infinite simple, finitary linear group and that $\mathcal{J} K^{t}[N]=\mathcal{J} K^{t}\left[\mathbb{S}^{p}(N)\right] \cdot K^{t}[N]$. Then $\mathcal{J} K^{t}[G]=\mathcal{J} K^{t}\left[\mathbb{S}^{p}(G)\right] \cdot K^{t}[G]$.

Proof. We know that $\mathcal{J} K^{t}\left[\mathbb{S}^{p}(G)\right] \cdot K^{t}[G] \subseteq \mathcal{J} K^{t}[G]$, so our goal is to prove the reverse inclusion when $\mathcal{J} K^{t}[G] \neq 0$. For this, let us first assume that $G / N$ is countably infinite and let $\alpha$ be a minimal element of $\mathcal{J} K^{t}[G]$ having 1 in its support. We show that $\alpha \in \mathcal{J} K^{t}\left[\mathbb{S}^{p}(G)\right] \cdot K^{t}[G]$.

To start with, if $\langle\operatorname{supp} \alpha\rangle \subseteq N$, then $\alpha \in \mathcal{J} K^{t}[G] \cap K^{t}[N] \subseteq \mathcal{J} K^{t}[N]$. Furthermore, since $\mathbb{S}^{p}(N) \triangleleft \mathbb{S}^{p}(G)$, we have $\mathcal{J} K^{t}\left[\mathbb{S}^{p}(N)\right] \subseteq \mathcal{J} K^{t}\left[\mathbb{S}^{p}(G)\right]$ and hence $\alpha \in \mathcal{J} K^{t}[N] \subseteq \mathcal{J} K^{t}\left[\mathbb{S}^{p}(G)\right] \cdot K^{t}[G]$, as required. Thus, we may suppose that $\langle\operatorname{supp} \alpha\rangle \nsubseteq N$, and we apply Lemma 11.2 to $\alpha$. In particular, let $H=H^{p}$ be the finite group given by that lemma, and let $L=H^{[G]}$ be the local subnormal closure of $H$ in $G$. If $C=N \cap L \triangleleft L$, then we claim that $(L, C, H)$ is a critical triple, as defined in Section 6 .

Of course, $H=H^{p}$ and $L=H^{L}$, so condition (2) is satisfied. Next, if we let $\sim: G \rightarrow G / N$ denote the natural epimorphism, then Lemma 4.5(v) implies that $\tilde{L}=\tilde{H}^{[\tilde{G}]}$. But $\tilde{H} \neq 1$ and $\tilde{G}$ is an infinite simple, finitary linear group, so $\tilde{H}^{[\tilde{G}]}=\tilde{G}$ by Lemma 5.6. Thus $G=N L$, so $L / C=L /(N \cap L) \cong N L / N=G / N$ is infinite simple and condition (1) holds. For (3), we see that $H \subseteq \mathbb{S}(N H)$ by Lemma 11.2(iv), and hence $H \subseteq \mathbb{S}(N H) \cap C H \subseteq \mathbb{S}(C H)$ by Lemma 4.1(iii). Next, observe that $\mathbb{F}(N)=\mathbb{F}(G)$ since $G / N$ is infinite simple, and hence $\mathbb{F}(L) \subseteq \mathbb{F}(G)=$ $\mathbb{F}(N)$ by Lemma 4.6(ii), since $L=H^{[G]}$. In particular, since $\mathbb{O}_{p}(G)=1$, it follows that $\mathbb{O}_{p}(L)=1$. Finally, by Lemma 11.2 (iv) again, we have $H \subseteq \mathbb{D}_{G}(\mathbb{F}(N)) \subseteq$ $\mathbb{D}_{G}(\mathbb{F}(L))$, since $\mathbb{F}(L) \subseteq \mathbb{F}(N)$. Thus, condition (3) is proved and $(L, C, H)$ is indeed a critical triple for the prime $p$. Furthermore, $L / C \cong G / N$ is countably infinite.

Now $\alpha \in \mathcal{J} K^{t}[G] \cap K^{t}[L] \subseteq \mathcal{J} K^{t}[L]$ and, by Proposition 10.3, we know that $\mathcal{J} K^{t}[L]=\mathcal{J} K^{t}[S] \cdot K^{t}[L]$, where $S=\mathbb{S}^{p}(L)$. As usual, this implies that $\pi_{S}(\alpha)$ is a nonzero truncation of $\alpha$ contained in $\mathcal{J} K^{t}[S] \subseteq \mathcal{J} K^{t}[L]$, and hence $\pi_{S}(\alpha) \in$ $\mathcal{J} K^{t}[L] \cap K^{t}[H] \subseteq \mathcal{J} K^{t}[H]$. The minimal nature of $\alpha$, as in Lemma 11.2(ii), now yields $\alpha=\pi_{S}(\alpha)$, so $\langle\operatorname{supp} \alpha\rangle \subseteq S=\mathbb{S}^{p}(L) \subseteq \mathbb{S}^{p}(G)$, by Lemma 4.6(i). Thus $\alpha \in \mathcal{J} K^{t}[G] \cap K^{t}\left[\mathbb{S}^{p}(G)\right] \subseteq \mathcal{J} K^{t}\left[\mathbb{S}^{p}(G)\right]$, as required. 
In other words, we have shown that every minimal element of $\mathcal{J} K^{t}[G]$ having 1 in its support is contained in $\mathcal{J} K^{t}\left[\mathbb{S}^{p}(G)\right] \cdot K^{t}[G]$ and therefore we conclude from Lemma 11.1 that $\mathcal{J} K^{t}[G] \subseteq \mathcal{J} K^{t}\left[\mathbb{S}^{p}(G)\right] \cdot K^{t}[G]$. This, of course, proves the result when $G / N$ is countably infinite.

For the general case, let $\beta$ be any element of $\mathcal{J} K^{t}[G]$. Since $G / N$ is an infinite simple group, it follows from [KWe, Theorem 4.4] that there is a subgroup $G_{0}$ of $G$ containing $\operatorname{supp} \beta$ with $N \subseteq G_{0}$ and with $G_{0} / N$ a countably infinite simple group. Of course, $G_{0} / N \subseteq G / N$, so $G_{0} / N$ is also a finitary linear group and therefore, by the above, $\mathcal{J} K^{t}\left[G_{0}\right]=\mathcal{J} K^{t}\left[\mathbb{S}^{p}\left(G_{0}\right)\right] \cdot K^{t}[G]$. Furthermore, since $G_{0} / N$ is infinite simple, it follows from Lemma $4.1\left(\right.$ iv) that $\mathbb{S}\left(G_{0} / N\right)=1$ and thus $\mathbb{S}^{p}\left(G_{0}\right)=\mathbb{S}^{p}(N)$, and similarly $\mathbb{S}^{p}(G)=\mathbb{S}^{p}(N)$. Finally, we have

$$
\begin{aligned}
\beta \in \mathcal{J} K^{t}[G] \cap K^{t}\left[G_{0}\right] & \subseteq \mathcal{J} K^{t}\left[G_{0}\right] \\
& =\mathcal{J} K^{t}\left[\mathbb{S}^{p}(N)\right] \cdot K^{t}\left[G_{0}\right] \subseteq \mathcal{J} K^{t}\left[\mathbb{S}^{p}(G)\right] \cdot K^{t}[G],
\end{aligned}
$$

and the result follows.

We now record an elementary observation.

Lemma 11.4. If $K^{t}[G]$ is given, then

$$
\mathcal{J} K^{t}\left[\mathbb{S}^{p}(G)\right]=\bigcup_{A} \mathcal{J} K^{t}[A],
$$

where the union is over all $A \operatorname{lsn} G$ with $A=A^{p}$. In particular, if $H$ is a finite subgroup of $G$ and $L=H^{[G]}$, then $\mathcal{J} K^{t}\left[\mathbb{S}^{p}(L)\right] \subseteq \mathcal{J} K^{t}\left[\mathbb{S}^{p}(G)\right]$.

Proof. If $A \operatorname{lsn} G$ with $A=A^{p}$, then $A \operatorname{lsn} \mathbb{S}^{p}(G)$ so $\mathcal{J} K^{t}[A] \subseteq \mathcal{J} K^{t}\left[\mathbb{S}^{p}(G)\right]$ by Lemma 7.4. Conversely, if $\beta \in \mathcal{J} K^{t}\left[\mathbb{S}^{p}(G)\right]$, then Lemma 4.1(ii) implies that there exists $B \operatorname{lsn} G$ with $B=B^{p}$ and $\operatorname{supp} \beta \subseteq B$. But then $\beta \in \mathcal{J} K^{t}\left[\mathbb{S}^{p}(G)\right] \cap K^{t}[B] \subseteq$ $\mathcal{J} K^{t}[B]$, so the reverse inclusion is proved. Finally, if $H$ and $L$ are as given, then the inclusion $\mathcal{J} K^{t}\left[\mathbb{S}^{p}(L)\right] \subseteq \mathcal{J} K^{t}\left[\mathbb{S}^{p}(G)\right]$ follows immediately from the above since Lemma 4.5(iii) asserts that any locally subnormal subgroup of $L$ is locally subnormal in $G$.

We close this section with a reasonable approximation to our main theorem. As expected, its proof requires the main result of [P13].

Lemma 11.5. Let $G$ be a locally finite group and let $K$ be an algebraically closed field of characteristic $p>0$. If $\mathbb{O}_{p}(G)=1$, then $\mathcal{J} K^{t}[G]=\mathcal{J} K^{t}\left[\mathbb{S}^{p}(G)\right] \cdot K^{t}[G]$.

Proof. We know that $\mathcal{J} K^{t}\left[\mathbb{S}^{p}(G)\right] \cdot K^{t}[G] \subseteq \mathcal{J} K^{t}[G]$, so we only need to prove the reverse inclusion. Of course, we can assume that $\mathcal{J} K^{t}[G] \neq 0$. Let $\alpha$ be a minimal element of $\mathcal{J} K^{t}[G]$ having 1 in its support, and let $H$ be the finite group given by Lemma 11.2. In particular, $H=H^{p}$ and $H=\langle\operatorname{supp} \alpha\rangle^{H}$. Now set $L=H^{[G]}$, so $L=$ $H^{L}$ and hence $L=\langle\operatorname{supp} \alpha\rangle^{L}$ by the above. Since $\mathbb{S}(L) \subseteq \mathbb{S}(G)$, by Lemma 4.6(i), we know that $\mathbb{O}_{p}(L)=1$ and hence $\mathbb{O}_{p}(N)=1$ where $N=\mathbb{S}^{p}(L)$. Now $N=\mathbb{S}(N)$, so Lemma 11.2(iv) implies that $H \subseteq \mathbb{D}_{L}\left(F^{*}\right) \triangleleft L$, where $F^{*}=\mathbb{F}^{*}(N)$. Indeed, since $L=H^{L}$, we have $L \subseteq \mathbb{D}_{L}\left(F^{*}\right)$, and hence $L$ acts in a strongly finitary manner on $F^{*}$ with kernel $C=\mathbb{C}_{L}\left(F^{*}\right)$. Note that $F^{*}$ is an f.c. group by Lemma 4.4 , so it follows from Proposition 5.5 that $L$ has a finite subnormal series

$$
C=L_{0} \triangleleft L_{1} \triangleleft \cdots \triangleleft L_{m}=L
$$


with each quotient $L_{i} / L_{i-1}$ either an f.c. group or an infinite simple, finitary linear group.

Suppose $A \operatorname{lsn} C$ with $A=A^{p}$. Then $A \operatorname{lsn} L$, so $A \subseteq N=\mathbb{S}^{p}(L)$ and since $A$ centralizes $F^{*}=\mathbb{F}^{*}(N)$, it follows from Lemma 4.3(ii) that $A \subseteq \mathbb{Z}(\mathbb{F}(N)$. But $\mathbb{O}_{p}(N)=1$ and $A=A^{p}$, so we must have $A=1$ and $\mathbb{S}^{p}(C)=1$. By the main result of [P13], we can now conclude that

$$
0=\mathcal{J} K^{t}[C]=\mathcal{J} K^{t}\left[\mathbb{S}^{p}(C)\right] \cdot K^{t}[C] .
$$

In other words, $K^{t}[C]$ satisfies the conclusion of our main theorem. Furthermore, since $\mathbb{O}_{p}\left(L_{i}\right)=1$, we can now use either Proposition 8.5 or Proposition 11.3 to prove, by induction, that each $K^{t}\left[L_{i}\right]$ also satisfies the conclusion of our main theorem. In particular, when $i=m$, we have $\mathcal{J} K^{t}[L]=\mathcal{J} K^{t}\left[\mathbb{S}^{p}(L)\right] \cdot K^{t}[L]$. Finally, we note that $\alpha \in \mathcal{J} K^{t}[G] \cap K^{t}[L] \subseteq \mathcal{J} K^{t}[L]$, and thus the preceding lemma yields

$$
\alpha \in \mathcal{J} K^{t}\left[\mathbb{S}^{p}(L)\right] \cdot K^{t}[L] \subseteq \mathcal{J} K^{t}\left[\mathbb{S}^{p}(G)\right] \cdot K^{t}[G] .
$$

Indeed, since this holds for all such $\alpha$, we conclude from Lemma 11.1 that $\mathcal{J} K^{t}[G] \subseteq$ $\mathcal{J} K^{t}\left[\mathbb{S}^{p}(G)\right] \cdot K^{t}[G]$, and the result follows.

\section{The Main Results}

In this final section, we state and prove the main theorems. As will be apparent, there is very little left to do. We just require a few observations to enable us to deal with normal $p$-subgroups and to obtain controller subgroup information. As usual, $K$ will denote a field of characteristic $p>0$ and, unless otherwise indicated, all groups considered will be locally finite.

Lemma 12.1. If $P$ is a locally finite $p$-group, then $K^{t}[P] / \mathcal{J} K^{t}[P]$ is a purely inseparable field extension of $K$.

Proof. Let $F$ be the algebraic closure of $K$ and let $F \otimes_{K} K^{t}[P]=F^{t}[P]$. Since $P$ is a locally finite $p$-group, it follows that $F^{t}[P] \cong F[P]$ (see for example [P13, Lemma 3.2(i)]). In particular, if $I$ denotes the augmentation ideal of $F^{t}[P]$, then $I$ is a nil ideal and $F^{t}[P] / I \cong F$. Now let $J=I \cap K^{t}[P]$ so that $J$ is a nil ideal of $K^{t}[P]$. Furthermore, since $\tilde{K}=K^{t}[P] / I$ is a $K$-subalgebra of $F^{t}[P] / I \cong F$, it follows that $\tilde{K}$ is an algebraic field extension of $K$. Indeed, since $\bar{P} K^{\bullet} / K^{\bullet}$ is a $p$-group, we see that $\tilde{K} / K$ is purely inseparable. Finally, since $J$ is a nil ideal and $K^{t}[P] / J$ is a field, we conclude that $J=\mathcal{J} K^{t}[P]$.

It is convenient to use some crossed product notation in the next two lemmas. While we do not require part (ii) below in its full generality, the result is certainly interesting, informative and worth stating.

Lemma 12.2. Let $G$ be a finite group.

i. If $R * G$ is a crossed product which is von Neumann regular and if $H$ is a subgroup of $G$, then $R * H$ is also von Neumann regular.

ii. Assume that the twisted group algebra $K^{t}[G]$ is semisimple. Then $G=N P$, where $N$ is a normal p-complement and where $P$ is an abelian Sylow $p$ subgroup with $K^{t}[P]$ a purely inseparable field extension of $K$. In particular, if either $K^{t}[G] \cong K[G]$ is an ordinary group algebra or $K$ is a perfect field, then $P=1$ and $G$ is a $p^{\prime}$-group. 
Proof. (i) Let $\pi_{H}: R * G \rightarrow R * H$ denote the usual $R * H$-bimodule projection. Now suppose that $R * G$ is von Neumann regular and let $\alpha \in R * H$. Then, by definition, there exists $\beta \in R * G$ with $\alpha \beta \alpha=\alpha$ and, by applying $\pi_{H}$ to this equation, we obtain $\alpha=\pi_{H}(\alpha)=\pi_{H}(\alpha \beta \alpha)=\alpha \cdot \pi_{H}(\beta) \cdot \alpha$. Since $\pi_{H}(\beta) \in R * H$, it follows that $R * H$ is also von Neumann regular.

(ii) Now let $P$ be a Sylow $p$-subgroup of $G$ and assume that $K^{t}[G]$ is semisimple and hence von Neumann regular. Then, by (i) above, $K^{t}[P]$ is also von Neumann regular and hence semisimple. In view of the preceding lemma, this implies that $K^{t}[P]$ is a purely inseparable field extension of $K$. In particular, $K^{t}[P]$ is commutative and $P$ is abelian. Furthermore, $\mathbb{N}_{G}(P)$ acts on $K^{t}[P]$ by conjugation and, of course, it centralizes $K$. Thus, since a purely inseparable field extension admits no nontrivial field automorphisms, we see that $\mathbb{N}_{G}(P)$ centralizes $K^{t}[P]$ and hence $P$. In other words, $P$ is in the center of its normalizer, and [Sc, Theorem 6.2.9] implies that $G$ has a normal $p$-complement $N$. Finally, if either $K^{t}[P] \cong K[P]$ or $K$ is a perfect field, then the preceding property of $K^{t}[P]$ implies that $K^{t}[P]=K$ and hence that $P=1$.

Next, we consider controlling subgroups in the finite situation.

Lemma 12.3. Let $G$ be a finite group and let $N \triangleleft G$ with $\mathcal{J} K^{t}[G]=\mathcal{J} K^{t}[N] \cdot K^{t}[G]$. If either $K^{t}[G] \cong K[G]$ is an ordinary group algebra or $K$ is a perfect field, then we must have $N \supseteq G^{p}$.

Proof. The case of ordinary group algebras is quite simple and we consider it first. Let $I$ denote the augmentation ideal of $K[N]$, so that $K[N] / I \cong K$ and $I \supseteq \mathcal{J} K[N]$. Since $K[G] /(\mathcal{J} K[N] \cdot K[G])$ is a finite dimensional semisimple algebra, it follows that its homomorphic image $K[G] /(I \cdot K[G]) \cong K[G / N]$ is also semisimple. Thus, by Lemma 12.2(ii), we see that $G / N$ is a $p^{\prime}$-group and hence $N \supseteq G^{p}$, as required.

Next we move on to the twisted case, but we assume that $K$ is an algebraically closed field. Let $P$ be a Sylow $p$-subgroup of $G$ and set $Q=P \cap N$, so that $Q$ is a Sylow $p$-subgroup of $N \triangleleft G$. The goal is to show that $P \subseteq N$ or equivalently that $P=Q$. To this end, we use crossed product notation and write $K^{t}[G]=$ $K^{t}[N] *(G / N)=R * \tilde{G}$, where $R=K^{t}[N]$ and ${ }^{\sim}: G \rightarrow G / N$ denotes the natural map. By assumption, $\mathcal{J}(R * \tilde{G})=J * \tilde{G}$ where $J=\mathcal{J} R$, and thus $(R * \tilde{G}) /(J * \tilde{G}) \cong$ $(R / J) * \tilde{G}$ is a finite dimensional semisimple algebra. Lemma 12.2(i) now implies that $(R / J) * \tilde{P}$ is also semisimple.

By Lemma 12.1, $K^{t}[P] \cong K[P]$ and, in particular, we can let $W$ denote the principal module for $K^{t}[Q] \cong K[Q]$. Since conjugation by $P$ stabilizes $W$, it follows that $P$ acts via conjugation on the induced $K^{t}[N]$-module $W^{N}=W \otimes_{K^{t}[Q]} K^{t}[N]$. Hence $P$ permutes the $K^{t}[N]$-composition factors of $W^{N}$. But $\operatorname{dim}_{K} W^{N}=|N: Q|$ is prime to $p$ and $P$ is a finite $p$-group, so we conclude that $P$ must stabilize some composition factor $V$. In other words, there exists an irreducible $K^{t}[N]$-module $V$ whose annihilator $I=\left\{\alpha \in K^{t}[N] \mid V \alpha=0\right\}$ is stabilized by $P$.

In particular, $I$ is a $\tilde{P}$-stable primitive ideal of $R$, so $I \supseteq \mathcal{J} R=J$ and hence $(R / I) * \tilde{P} \cong(R * \tilde{P}) /(I * \tilde{P})$ is a homomorphic image of the finite dimensional semisimple algebra $(R / J) * \tilde{P}$. Thus, $(R / I) * \tilde{P}$ is also semisimple. Furthermore, $K$ is algebraically closed, so $R / I \cong M_{n}(K)$ for some $n \geq 1$ and hence all $K$-automorphisms of $R / I$ are inner. It follows that

$$
(R / I) * \tilde{P} \cong M_{n}(K) * \tilde{P} \cong M_{n}(K) \otimes_{K} K^{t^{\prime}}[\tilde{P}] \cong M_{n}\left(K^{t^{\prime}}[\tilde{P}]\right)
$$


where $K^{t^{\prime}}[\tilde{P}]$ is a suitable twisted group algebra of $\tilde{P}$. But $(R / I) * \tilde{P}$ is semisimple, so $K^{t^{\prime}}[\tilde{P}]$ is also semisimple, and we conclude from Lemma 12.1 that $\tilde{P}=1$. Hence, $P \subseteq N$, as required.

Finally, let $K$ be a perfect field and let $F$ be its algebraic closure. Then $F^{t}[G]=$ $F \otimes_{K} K^{t}[G]$, so $\left[\mathrm{P} 5\right.$, Theorem 7.2.13] implies that $\mathcal{J} F^{t}[G]=F \otimes_{K} \mathcal{J} K^{t}[G]$ and $\mathcal{J} F^{t}[N]=F \otimes_{K} \mathcal{J} K^{t}[N]$. Consequently, the equality $\mathcal{J} K^{t}[G]=\mathcal{J} K^{t}[N] \cdot K^{t}[G]$ translates to $\mathcal{J} F^{t}[G]=\mathcal{J} F^{t}[N] \cdot F^{t}[G]$, so we conclude from the preceding special case that $N \supseteq G^{p}$, and the result follows.

As a consequence, we have

Lemma 12.4. Let $G$ be an arbitrary group, let $I$ be a nil ideal of $K^{t}[G]$ and assume that $N \triangleleft G$ controls $I$. Furthermore, suppose that either $K^{t}[G] \cong K[G]$ is an ordinary group algebra or that $K$ is a perfect field. If $H$ is a finite subgroup of $G$ with $\mathcal{J} K^{t}[H] \subseteq I$, then $H^{p} \subseteq N$.

Proof. Set $M=H \cap N \triangleleft H$ and note that $\mathcal{J} K^{t}[H] \subseteq I$ by assumption. Since $I=\left(I \cap K^{t}[N]\right) \cdot K^{t}[G]$, it follows that $\pi_{N}(I)=I \cap K^{t}[N] \subseteq I$ and hence $\pi_{N}(I)$ is a nil ideal of $K^{t}[N]$. In particular, $\pi_{M}\left(\mathcal{J} K^{t}[H]\right)=\pi_{N}\left(\mathcal{J} K^{t}[H]\right)$ is a nil ideal of $K^{t}[M]$. Thus $\pi_{M}\left(\mathcal{J} K^{t}[H]\right) \subseteq \mathcal{J} K^{t}[M]$, and we conclude from Lemma 7.10 that $\mathcal{J} K^{t}[H] \subseteq \mathcal{J} K^{t}[M] \cdot K^{t}[H]$. Since the reverse inclusion always holds by Lemma 7.2, the preceding lemma applied to $M \triangleleft H$ yields $H^{p} \subseteq M \subseteq N$.

As promised in Section 1, we can now prove

Proposition 12.5. Let $K^{t}[G]$ be a twisted group algebra of the arbitrary group $G$ over a field $K$ of characteristic $p>0$. If either $K^{t}[G] \cong K[G]$ is an ordinary group algebra or $K$ is a perfect field, then the controller of the nilpotent radical $\mathcal{N} K^{t}[G]$ is equal to $\Delta^{p}(G)$.

Proof. By Proposition 1.1, we know that $\mathcal{C}\left(\mathcal{N} K^{t}[G]\right) \subseteq \Delta^{p}(G)$. Furthermore, $I=$ $\mathcal{N} K^{t}[G]$ is a nil ideal and $\mathcal{J} K^{t}[D] \subseteq I$ for all finite normal subgroups $D$ of $G$ with $D=D^{p}$. Thus, the preceding lemma implies that $D=D^{p} \subseteq \mathcal{C}(I)$, so $\mathcal{C}(I)$ contains $\Delta^{p}(G)$, the subgroup of $G$ generated by all such $D$.

The following is our main result on twisted group algebras $K^{t}[G]$ when $\mathbb{O}_{p}(G)$ is trivial. Parts (i) through (iv) constitute the statement of Theorem 1.2, while part (v) discusses the controller subgroup.

Theorem 12.6. Let $K^{t}[G]$ be a twisted group algebra of the locally finite group $G$ over a field $K$ of characteristic $p>0$. If $\mathbb{O}_{p}(G)=1$, then

i. $\mathcal{J} K^{t}[G]=\mathcal{J} K^{t}\left[\mathbb{S}^{p}(G)\right] \cdot K^{t}[G]$.

ii. $\mathcal{J} K^{t}\left[\mathbb{S}^{p}(G)\right]=\bigcup_{A \in \mathfrak{S}} \mathcal{J} K^{t}[A]$, where $\mathfrak{S}$ is the set of all locally subnormal subgroups $A$ of $G$ with $A=A^{p}$.

iii. $\mathcal{J} K^{t}[G]=0$ if and only if $\mathcal{J} K^{t}[A]=0$ for all $A \in \mathfrak{S}$. In particular, if $\mathfrak{S}$ consists of just the identity group 1 , then $\mathcal{J} K^{t}[G]=0$.

iv. $\mathcal{J} K^{t}[G]$ is a prime ideal of $K^{t}[G]$ when $G$ has no nonidentity finite normal subgroups.

v. $\mathcal{C}\left(\mathcal{J} K^{t}[G]\right)=\mathbb{S}^{p}(G)$ if either $K^{t}[G] \cong K[G]$ is an ordinary group algebra or $K$ is a perfect field.

Proof. Let $F$ be the algebraic closure of $K$ and set $F^{t}[G]=F \otimes_{K} K^{t}[G]$. By [P5, Theorem 7.2.13], we have $\mathcal{J} K^{t}[G] \subseteq \mathcal{J} F^{t}[G]$ and, by Lemma 11.5, we know that 
$\mathcal{J} F^{t}[G]=\mathcal{J} F^{t}\left[\mathbb{S}^{p}(G)\right] \cdot F^{t}[G]$. In particular, if $S=\mathbb{S}^{p}(G)$ and $\pi_{S}: F^{t}[G] \rightarrow F^{t}[S]$ is the natural projection, then $\pi_{S}\left(\mathcal{J} K^{t}[G]\right) \subseteq \pi_{S}\left(\mathcal{J} F^{t}[G]\right) \subseteq \mathcal{J} F^{t}[S]$ is nil by Lemma 7.1. In other words, $\pi_{S}\left(\mathcal{J} K^{t}[G]\right)$ is a nil ideal of $K^{t}[S]$, so we can conclude from Lemma 7.10 that

$$
\mathcal{J} K^{t}[G] \subseteq \pi_{S}\left(\mathcal{J} K^{t}[G]\right) \cdot K^{t}[G] \subseteq \mathcal{J} K^{t}[S] \cdot K^{t}[G] .
$$

Since the reverse inclusion always holds, by Lemma 7.3, part (i) is proved.

Note that part (ii) follows from Lemma 11.4, and then (iii) is obvious. Furthermore, part (iv) is an immediate consequence of (i) and Proposition 8.4. Finally, if $C=\mathcal{C}\left(\mathcal{J} K^{t}[G]\right)$, then (i) implies that $C \subseteq \mathbb{S}^{p}(G)$. On the other hand, $\mathcal{J} K^{t}[G]$ is a nil ideal and $\mathcal{J} K^{t}[A] \subseteq \mathcal{J} K^{t}[G]$ for all $A$ lsn $G$ with $A=A^{p}$. Thus, if either $K^{t}[G] \cong K[G]$ or $K$ is perfect, then $A=A^{p} \subseteq C$ by Lemma 12.4, and hence $C \supseteq \mathbb{S}^{p}(G)$, as required.

The general case follows easily from the above by modding out by $\mathbb{O}_{p}(G)$. Indeed, part (i) below yields the essential structure, while (ii) and (iii) constitute the statement of Corollary 1.3. Recall that the group $\mathbb{T}^{p}(G) \supseteq \mathbb{O}_{p}(G)$ is defined by

$$
\mathbb{T}^{p}(G) / \mathbb{O}_{p}(G)=\mathbb{S}^{p}\left(G / \mathbb{O}_{p}(G)\right)
$$

The following is our main result on the Jacobson radical of twisted group algebras of arbitrary locally finite groups.

Corollary 12.7. Let $K^{t}[G]$ be a twisted group algebra of the locally finite group $G$ over a field $K$ of characteristic $p>0$. If $P=\mathbb{O}_{p}(G)$ and if $F=K^{t}[P] / \mathcal{J} K^{t}[P]$, then $F$ is a purely inseparable field extension of $K$, and we have

i. $\mathcal{J} K^{t}[P] \cdot K^{t}[G] \subseteq \mathcal{J} K^{t}[G]$ and

$$
K^{t}[G] /\left(\mathcal{J} K^{t}[P] \cdot K^{t}[G]\right) \cong F^{t}[G / P],
$$

where the latter is a suitable twisted group algebra of $G / P$ over $F$.

ii. $\mathcal{J} K^{t}[G]=\mathcal{J} K^{t}\left[\mathbb{T}^{p}(G)\right] \cdot K^{t}[G]$.

iii. $\mathcal{J} K^{t}[G]=0$ if and only if $\mathcal{J} K^{t}[A]=0$ for all locally subnormal subgroups $A$ of $G$ with $A=A^{p}$. In particular, if the only such group $A$ is the identity 1 , then $\mathcal{J} K^{t}[G]=0$.

iv. $\mathcal{J} K^{t}[G]$ is a prime ideal of $K^{t}[G]$ when $G / P$ has no nontrivial finite normal subgroups.

v. $\mathcal{C}\left(\mathcal{J} K^{t}[G]\right)=\mathbb{T}^{p}(G)$ if either $K^{t}[G] \cong K[G]$ is an ordinary group algebra or $K$ is a perfect field.

Proof. (i) For convenience, write $K^{t}[G]=R * \tilde{G}$ where $R=K^{t}[P]$ and ${ }^{\sim}: G \rightarrow$ $G / P$. If $J=\mathcal{J} R=\mathcal{J} K^{t}[P]$, then $K^{t}[G] /\left(J \cdot K^{t}[G]\right)=(R * \tilde{G}) /(J * \tilde{G}) \cong(R / J) * \tilde{G}$. Furthermore, note that $R / J=F$ is a purely inseparable field extension of $K$, by Lemma 12.1. Hence, since $F$ admits no nontrivial $K$-algebra automorphisms, it follows that $F$ is central in $(R / J) * \tilde{G}$ and therefore $K^{t}[G] /\left(J \cdot K^{t}[G]\right) \cong F * \tilde{G} \cong$ $F^{t}[\tilde{G}]$. Since $J \cdot K^{t}[G] \subseteq \mathcal{J} K^{t}[G]$ by Lemma 7.3 , the first part is proved.

(ii) Let $\chi: K^{t}[G] \rightarrow F^{t}[\tilde{G}]$ denote the natural ring epimorphism given above with $\operatorname{ker} \chi=\mathcal{J} K^{t}[P] \cdot K^{t}[G]$. Set $I_{1}=\mathcal{J} K^{t}[G]$ and $I_{2}=\mathcal{J} K^{t}\left[\mathbb{T}^{p}(G)\right] \cdot K^{t}[G]$, and note that these ideals contain ker $\chi$ by Lemma 7.3. Furthermore, by Theorem 12.6(i), we have

$$
\chi\left(I_{1}\right)=\mathcal{J} F^{t}[\tilde{G}]=\mathcal{J} F^{t}\left[\mathbb{S}^{p}(\tilde{G})\right] \cdot F^{t}[\tilde{G}]=\chi\left(I_{2}\right),
$$

and therefore $I_{1}=I_{2}$, as required. 
(iii) If $\mathcal{J} K^{t}[A] \neq 0$ for some $A \operatorname{lsn} G$ with $A=A^{p}$, then $\mathcal{J} K^{t}[G] \neq 0$ by Lemma 7.4. Conversely, let us suppose that $\mathcal{J} K^{t}[G] \neq 0$. If $\mathcal{J} K^{t}[P] \neq 0$, then since $P=\mathbb{S}^{p}(P)$, it follows from Lemma 11.4 that there exists $A \operatorname{lsn} P$ with $\mathcal{J} K^{t}[A] \neq 0$. But $P \triangleleft G$, so $A$ lsn $G$ and surely $A=A^{p}$. On the other hand, if $\mathcal{J} K^{t}[P]=0$, then $F=K^{t}[P]$ is central in $K^{t}[G]$ and hence $P$ is central in $G$. Furthermore, $K^{t}[G] \cong F^{t}[\tilde{G}]$, so Theorem 12.6(iii) implies that there exists $\tilde{B} \operatorname{lsn} \tilde{G}$ with $\tilde{B}=\tilde{B}^{p}$ and $\mathcal{J} F^{t}[\tilde{B}] \neq 0$. Now, if $\tilde{B}=B / P$, then $B$ is center-by-finite and hence it is an f.c. group. Thus, since $\mathcal{J} K^{t}[B] \cong \mathcal{J} F^{t}[\tilde{B}] \neq 0$ and $B=B^{p}$, we can find a finite normal subgroup $A$ of $B$ with $A=A^{p}$ and $0 \neq \mathcal{J} K^{t}[B] \cap K^{t}[A] \subseteq \mathcal{J} K^{t}[A]$. But $A \triangleleft B$ and $\tilde{B} \operatorname{lsn} \tilde{G}$, so it follows easily that $A \operatorname{lsn} G$.

(iv) This is immediate from part (i) and Theorem 12.6(iv).

(v) Here, the assumptions imply that $F=K$ and that $\mathcal{J} K^{t}[P]$ is the augmentation ideal of $K^{t}[P]$. By (i), we know at least that $C=\mathcal{C}\left(\mathcal{J} K^{t}[G]\right) \subseteq \mathbb{T}^{p}(G)$. For the converse, let $A$ be a finite subgroup of $P$. Then $A \operatorname{lsn} G$, so $\mathcal{J} K^{t}[A] \subseteq \mathcal{J} K^{t}[G]$ by Lemma 7.4. In particular, $A=A^{p} \subseteq C$ by Lemma 12.4, and hence $C \supseteq P$. Now note that

$$
\mathcal{J} K^{t}[G]=\left(\mathcal{J} K^{t}[G] \cap K^{t}[C]\right) \cdot K^{t}[G]=\mathcal{J} K^{t}[C] \cdot K^{t}[G]
$$

by Lemmas 7.1 and 7.3. Thus, since this ideal contains ker $\chi$, we see that

$$
\mathcal{J} F^{t}[\tilde{G}]=\chi\left(\mathcal{J} K^{t}[G]\right)=\chi\left(\mathcal{J} K^{t}[C] \cdot K^{t}[G]\right)=\mathcal{J} F^{t}[\tilde{C}] \cdot F^{t}[\tilde{G}]
$$

and therefore $\tilde{C}=C / P$ controls $\mathcal{J} F^{t}[\tilde{G}]$. Of course, if $K$ is a perfect field, then so is $F=K$. On the other hand, if $K^{t}[G] \cong K[G]$, then it is clear that $F^{t}[\tilde{G}] \cong F[\tilde{G}]$. Thus, in either case, we conclude from Theorem $12.6(\mathrm{v})$ that $\tilde{C} \supseteq \mathbb{S}^{p}(\tilde{G})$ and therefore that $C \supseteq \mathbb{T}^{p}(G)$.

We close this paper with two examples of interest. The first shows that the purely inseparable fields we have been discussing can actually occur and hence that the controller information we have obtained is best possible.

Lemma 12.8. Let $P$ be an abelian p-group.

i. There exist a field $K$ of characteristic $p>0$ and a twisted group algebra $K^{t}[P]$, such that $K^{t}[P]$ is a purely inseparable field extension of $K$. In particular, $\mathcal{J} K^{t}[P]=0$, so $\mathcal{C}\left(\mathcal{J} K^{t}[P]\right)=1$ is properly smaller than $\mathbb{T}^{p}(P)=P$.

ii. If $P$ is finite and acts faithfully on the finite $p^{\prime}$-group $N$, then there exist a field $K$ of characteristic $p$ and a twisted group algebra $K^{t}[G]$ for the semidirect product $G=N \rtimes P$ such that $\mathcal{J} K^{t}[G]=0$. In particular, $\mathbb{O}_{p}(G)=1$, but $\mathcal{C}\left(\mathcal{J} K^{t}[G]\right)=1$ is properly smaller than $\mathbb{S}^{p}(G)=G^{p}$.

Proof. Assume that $P$ is an abelian $p$-group which acts on the group $N$. Write $P=A / B$, where $A$ is a free abelian group, and form the semidirect product $G=$ $N \rtimes A$, where $A$ acts on $N$ via its homomorphism to $P$. Note that $B$ is a central subgroup of $G$ and that $G / B \cong N \rtimes P$.

Let $k$ be a field of characteristic $p>0$ and let $L=k[G]$ be the group algebra of $G$ over $k$. Since $B \triangleleft G$, we have $L=R *(G / B)$ where $R=k[B]$. Note also that $R$ is a central subring of $L$ and that it consists of regular elements of $L$ by freeness and [P5, Theorem 13.1.11]. Thus, we can form the ring of fractions $L R^{-1}$, and from the above crossed product formulation, it is clear that

$$
L R^{-1} \cong K *(G / B) \cong K^{t}[G / B] \cong K^{t}[N \rtimes P],
$$

where $K$ is the field $R R^{-1}$. 
(i) Here we take $N=1$ and observe that $L=k[A]$ is a commutative domain by [P5, Theorem 13.1.11] again. Thus $L R^{-1} \cong K^{t}[P]$ is a twisted group algebra which is a domain, algebraic over $K$. It follows that $K^{t}[P]$ is a field extension of $K$ which is purely inseparable since $P$ is a $p$-group.

(ii) Now we suppose that $N$ and $P$ are both finite and that $P$ acts faithfully on $N$. Since $N$ is a $p^{\prime}$-group, Proposition 1.1 implies that $L=k[N \rtimes A]$ is semiprime. Hence $L R^{-1} \cong K^{t}[N \rtimes P]$ is also semiprime, and therefore it is a finite dimensional semisimple $K$-algebra. Since $P$ acts faithfully on $N$ and since $\mathbb{O}_{p}(G)$ acts trivially on $N$, it follows that $\mathbb{O}_{p}(G)=1$.

Of course, in part (ii) above, we can take $P \cong Z_{p}$ to be cyclic of order $p$ and $N \cong Z_{q} \times Z_{q} \times \cdots \times Z_{q}$ to be the direct product of $p$ copies of $Z_{q}$ with $q$ a prime different from $p$. Then $P$ acts faithfully on $N$ by cyclically permuting its factors and if $G=N \rtimes P$, it is easy to see that $\left|G: G^{p}\right|=q$.

Finally, we offer an example to show that $\mathbb{T}^{p}(G)$ can be properly larger than its subgroup $\mathbb{S}^{p}(G)$. Note that $\mathbb{T}^{p}(G)=\mathbb{S}^{p}(G)$ when $\mathbb{O}_{p}(G)$ is finite, so the following construction necessarily requires $\mathbb{O}_{p}(G)$ to be infinite.

Lemma 12.9. Let $S$ be a finite nonabelian simple group of order divisible by $p$ and suppose that $S$ acts nontrivially on the finite abelian p-group $A$. Then $S$ acts naturally on the (weak) direct product $P=\prod_{i=1}^{\infty} A_{i}$ of infinitely many copies $A_{i}$ of $A$, and $G=P \rtimes S$ is a locally finite group with $\mathbb{S}^{p}(G)=\mathbb{O}_{p}(G)=P$ and with $\mathbb{T}^{p}(G)=G$.

Proof. It is clear that $P=\mathbb{O}_{p}(G) \subseteq \mathbb{S}^{p}(G) \subseteq \mathbb{T}^{p}(G)=G$ and thus there are only two possibilities for $\mathbb{S}^{p}(G)$. If $\mathbb{S}^{p}(G)=G$, then there exists $H \operatorname{lsn} G$ with $H \supseteq S$. Note that $H \cap P \triangleleft H$ and that $H \cap P \triangleleft P$ since $P$ is abelian. Thus $H \cap P \triangleleft G$ and if $\tilde{G}=G /(H \cap P)$, then $\tilde{H}=H /(H \cap P) \cong S$ is locally subnormal in $\tilde{G}$. But $\tilde{H}$ is quasi-simple, so the local version of Lemma 2.7 (ii) implies that $\tilde{H}$ centralizes $\tilde{P}$ and therefore $[P, S]=[P, H] \subseteq H \cap P$ is finite. But $[P, S]=\prod_{i=1}^{\infty}\left[A_{i}, S\right]$ is certainly infinite, so this case cannot occur and hence we must have $\mathbb{S}^{p}(G)=P$.

\section{REFERENCES}

[A] S. A. Amitsur, On the semi-simplicity of group algebras, Mich. Math. J. 6 (1959), 251-253. MR 21:7256

[Be] V. V. Belyaev, Locally finite Chevalley groups, Studies in Group Theory, Urals Scientific Centre of the Academy of Sciences of USSR, Sverdlovsk, 1984, pp. 39-50 (Russian). MR $\mathbf{8 7 d}: 20043$

[Bo] A. V. Borovik, Periodic linear groups of odd characteristic, Soviet Math. Dokl. 26 (1982), 484-486. MR 84d:20042

[DZ] Z. Z. Dyment and A. E. Zalesskiǔ, On the lower radical of a group ring, Dokl. Akad. Nauk BSSR 19 (1975), 876-879 (Russian). MR 52:5723

[FMo] J. W. Fisher and S. Montgomery, Semiprime skew group rings, J. Algebra 52 (1978), 241-247. MR 58:772

[H1] J. I. Hall, Locally finite simple groups of finitary linear transformations, Finite and Locally Finite Groups, Kluwer, Dordrecht, 1995, pp. 147-188. MR 96j:20042

[H2] , Periodic simple groups of finitary linear transformations (to appear).

[HaS] B. Hartley and G. Shute, Monomorphisms and direct limits of finite groups of Lie type, Quart. J. Math. Oxford (2) 35 (1984), 49-71. MR 85k:20094

[Hu] B. Huppert, Endliche Gruppen I, Springer-Verlag, Berlin, 1967. MR 37:302

[KWe] O. H. Kegel and B. A. F. Wehrfritz, Locally Finite Groups, North-Holland, Amsterdam, 1973. MR 57:9848

[M] U. Meierfrankenfeld, Non-finitary locally finite simple groups, Finite and Locally Finite Groups, Kluwer, Dordrecht, 1995, pp. 189-212. MR 97a:20066 
[Mo] S. Montgomery, Outer automorphisms of semiprime rings, J. London Math. Soc. (2) 18 (1978), 209-220. MR 80b:16028

[P1] D. S. Passman, Radicals of twisted group rings, Proc. London Math. Soc. (3) 20 (1970), 409-437. MR 42:6129

[P2] — A new radical for group rings?, J. Algebra 28 (1974), 556-572. MR 55:397

[P3] Subnormality in locally finite groups, Proc. London Math. Soc. (3) 28 (1974), 631-653. MR 50:13295

[P4] - Radical ideals in group rings of locally finite groups, J. Algebra 33 (1975), 472497. MR 52:10793

[P5] , The Algebraic Structure of Group Rings, Wiley-Interscience, New York, 1977. MR 81d: 16001

[P6] The Jacobson radical of a group ring of a locally solvable group, Proc. London Math. Soc. 38 (1979), 169-192, (ibid 39 (1979), 208-210). MR 81j:16017a,b

[P7] Infinite Crossed Products, Academic Press, Boston, 1989. MR 90g:16002

[P8] Semiprimitivity of group algebras of locally finite groups, Infinite Groups and Group Rings, World Scientific, Singapore, 1993, pp. 77-101. MR 97b:16029

[P9] , Semiprimitivity of group algebras of infinite simple groups of Lie type, Proc. Amer. Math. Soc. 121 (1994), 399-403. MR 94h:16039

[P10] - Semiprimitivity of group algebras of locally finite groups II, J. Pure Appl. Algebra 107 (1996), 271-302. MR 97b:16030

[P11] _ Semiprimitivity of group algebras: a survey, Atas da XII Escola de Álgebra, IMECC-UNICAMP, 1995, pp. 168-187.

[P12] _ The semiprimitivity problem for group algebras of locally finite groups, Israel J. Math. 96 (1996), 481-509.

[P13] - The semiprimitivity problem for twisted group algebras of locally finite groups, Proc. London Math. Soc. (3) 73 (1996), 323-357. CMP 96:15

[PZ] D. S. Passman and A. E. Zalesskiŭ, Semiprimitivity of group algebras of locally finite simple groups, Proc. London Math. Soc. 67 (1993), 243-276. MR 94g:16036

[Ph1] R. E. Phillips, Finitary linear groups: a survey, Finite and Locally Finite Groups, Kluwer, Dordrecht, 1995, pp. 111-146. MR 96m:20080

[Ph2] _ Primitive, locally finite, finitary linear groups, J. Algebra (to appear).

[Sc] W. R. Scott, Group Theory, Prentice-Hall, Englewood Cliffs, 1964. MR 29:4785

[T] S. Thomas, The classification of the simple periodic linear groups, Archiv der Mathematik 41 (1983), 103-116. MR 85f:20040b

[V] O. E. Villamayor, On the semisimplicity of group algebras, Proc. Amer. Math. Soc. 9 (1958), 621-627. MR 20:5224

[W1] H. Wielandt, Eine Verallgemeinerung der invarianten Untergruppen, Math. Z. 45 (1939), 209-244.

[W2] Unendliche Permutationsgruppen, Lecture Notes, Math. Inst. Univ. Tübingen, 1960.

[W3] Mathematische Werke, Volume 1, de Gruyter, Berlin, 1994. MR 95b:01025

[Za] H. Zassenhaus, The Theory of Groups, Second Edition, Chelsea, New York, 1958. MR 19:939d

Department of Mathematics, University of Wisconsin, Madison, Wisconsin 53706

E-mail address: passman@math.wisc.edu 